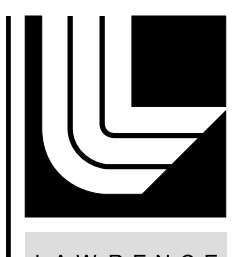

LAWRENCE LIVERMORE N A TIO N A L LABORATORY

\title{
UCRL-TR-214453
}

\section{CMS Annual Report 2004}

T. D. de la Rubia, S. P. Shang, G. Rennie, M.

Fluss, C. Westbrook

August 9, 2005 
This document was prepared as an account of work sponsored by an agency of the United States Government. Neither the United States Government nor the University of California nor any of their employees, makes any warranty, express or implied, or assumes any legal liability or responsibility for the accuracy, completeness, or usefulness of any information, apparatus, product, or process disclosed, or represents that its use would not infringe privately owned rights. Reference herein to any specific commercial product, process, or service by trade name, trademark, manufacturer, or otherwise, does not necessarily constitute or imply its endorsement, recommendation, or favoring by the United States Government or the University of California. The views and opinions of authors expressed herein do not necessarily state or reflect those of the United States Government or the University of California, and shall not be used for advertising or product endorsement purposes.

This work was performed under the auspices of the U.S. Department of Energy by University of California, Lawrence Livermore National Laboratory under Contract W-7405-Eng-48. 


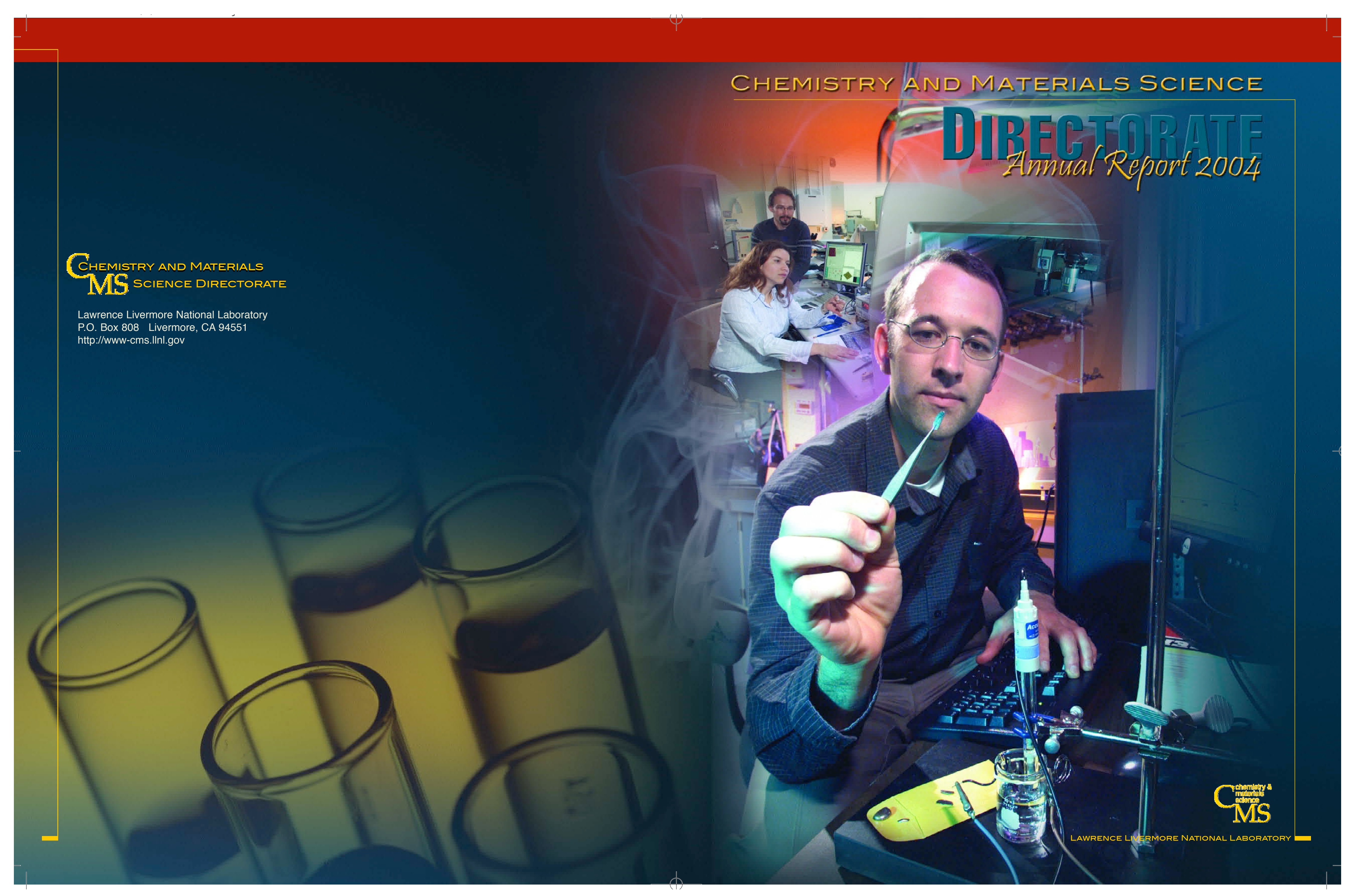


Sbout the Gover Formed in 2004 to exploit nanoscience and nanotechnol the Nanoscale Synthesis and Characterization Laboratory is a new center of excellence for developing novel nanostructure that will enable many

Gredits

Scientific Editors Michael Fluss

Charlie Westbrook

Production Editors Stephanie Shan

Contributing Editors Linda Null
Allan Francke

rt Director

George A. Kitrinos

Production Staff

Louisa Cardoza
George A. Kitrinos

Photographer

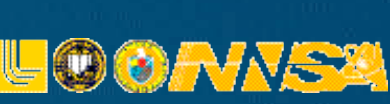

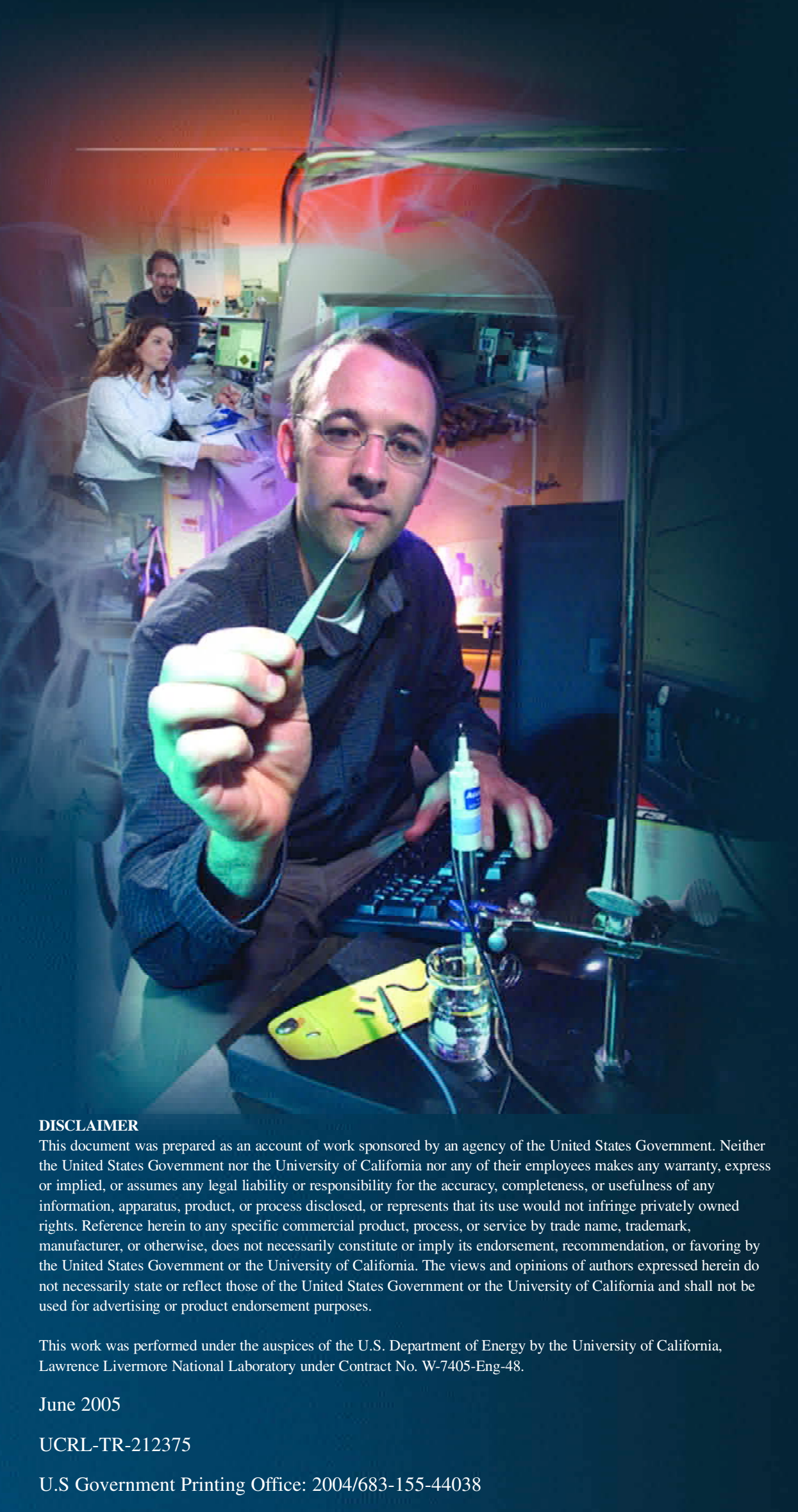

WHAT'S INSIDE CMS

A message from Tomás Díaz de la Rubia

$\begin{aligned} & \text { Science in support of national objectives at the } \\ & \text { intersection of chemistry, materials science, and biology } \\ & 8 \text { Unraveling the Molecular Modulation of Kidney Stone Formatio } \\ & 10 \text { Smart Membranes for Bio-Organism Detection } \\ & 12 \text { Molecular Transport in Carbon Nanotubes } \\ & 14 \text { Novel Approach for Identifying Bacterial Toxin Inhibitors }\end{aligned}$

Materials properties and performance under

extreme conditions

16 Synthesis and Characterization of Nanoporous Metal Foam

18 Unraveling the Mysteries of Dislocation Formation

$\begin{array}{ll}18 & \text { Unraveling the Mysteries of Dislocation Formation } \\ 20 & \text { Time-Resolved Observations of Electronic Structure }\end{array}$

22 Ultrafast Dynamics of Metal Deformation

24 Emergent Materials Properties of Putoniun

\footnotetext{
Chemistry under extreme conditions and chemical

engineering in support of national-security programs

26 Modeling the Many Faces of Water

28 Formation of Methane under the Earth's Mantle

30 Probing Materials under Extreme Conditions

32 Structure Control in Sol-Gel Materials
}

Applied nuclear science for human health and national security

34 Tracing Iodine-129 from Nuclear Fuel Reprocessing Facilities

36 Age-Dating Groundwater to Detect Contamination

38 Isotopic Composition of Interplanetary Dust Particles

Divisions, centers, and institutes

Materials Science and Technology Division

44 Chemistry and Chemical Engineering Division

46 Chemical Biology and Nuclear Science Division

48 BioSecurity and Nanosciences Laboratory

50 Energetic Materials Center

52 Forensic Science Center

54 Glenn T. Seaborg Institute

56 Center for National Security Applications of Magnetic Resonance

58 Nanoscale Synthesis and Characterization Laboratory

60 Postdoctoral Fellows and Graduate Students

62 Awards and Recognition in 2004 
Al message from Tomás Díaz de la Ritjes $G_{\text {transformation that is reshaping the landscape of materials }}^{\text {lance at the articles in }}$ science and chemistry. This transformation is bridging the gaps among chemistry, materials science, and biology - ushering in a wealth of innovative technologies with broad scientific impact. The emergence of this intersection is reinvigorating our strategic investment into areas that build on our strength of iner strategic vision into a future where we will provide rasical materials innovations and solutions to our national-security programs and other sponsors.

Our 2004 Annual Report describes how our successes and breakthroughs follow a path set forward by our strategic pla and four organizing research themes, each with key scientific accomplishments by our staff and collaborators.

We have organized this report into two major sections: research themes and our dynamic teams. The research-them sections focus on achievenents arising from earlier

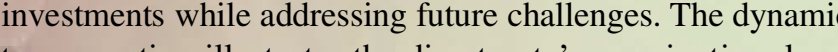
teans structure of divisions, centers, and instiontes the structure of divisions, centers, and institutes th support a team environment across
disciplinary and institutional boundaries.
The research presented in this annual The research presented in this annual report gives substantive examples of how we are proceeding in each of these four
theme areas and how they are aligned theme areas and how they are aligned with our national-secunty mission. By maintaining an organizational structure problem-solving oppotuities, we are able to nurture the discoveries and breakthroughs required for future successes.
Science Supporting National Objectives at the

Intersection of Chemistry, Materials Science, and Biology Many applications in nano- and biotechnology readily build on our unique mixture of disciplines, specifically at the intersection of chemistry, materials science, and biology. We

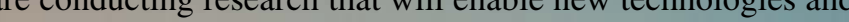
tools to combat chemical and biological terrorism and enhance far ental human-health research.

By manipulating mate laboratory-synthesized and carbon natubes, we continue to develop new materins, systems for advanced nanoscale sensors and detectors. Our studies on the molecular mechanisms of tissue mineralization may allow us to better define strategies for designing therapeutic drugs to treat diseases such as kidney stones. The discoveries also help us predict molecular interactions in complex

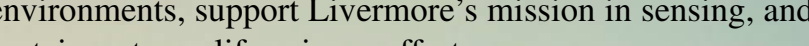
sustain a strong lite-scince elfor.

\section{Materials Properties and Perf ormance under}

\section{xtreme Conditions}

Our emerging ability to control matter on the nanometer cale is leading to many revolutionary applications. Succesfu synthesis of nanoporus materials with engineered properties is the first step toward entirely new types of novel materials that will find applications as critical components of future targets for the National Ignition Facility and other high-energy-density-

science platforms.
Unparalleled resources-both in computation and experiments-are required in order to observe the ultrafast dynamics of materials at the same scales of the underlying deformation, electronic structural changes, and quantum criticality offers insight into materials performance and, more importantly, their failure mechanisms.

\section{Chemistry under Extreme Conditions and Chemical} Engineering to Support National-Security Programs

Taking advantage of the immense computing resources a Lawrence Livermore, our scientists have unique tools to not only study exteme phentorion, but also to decipher the chemistry of planetary interiors and oth modeling of extreme chemistry is best illustrated in our latest simulation-based discoveries of superionic and polymeric phases of water and the origin of methane at extreme geophysical pressures.

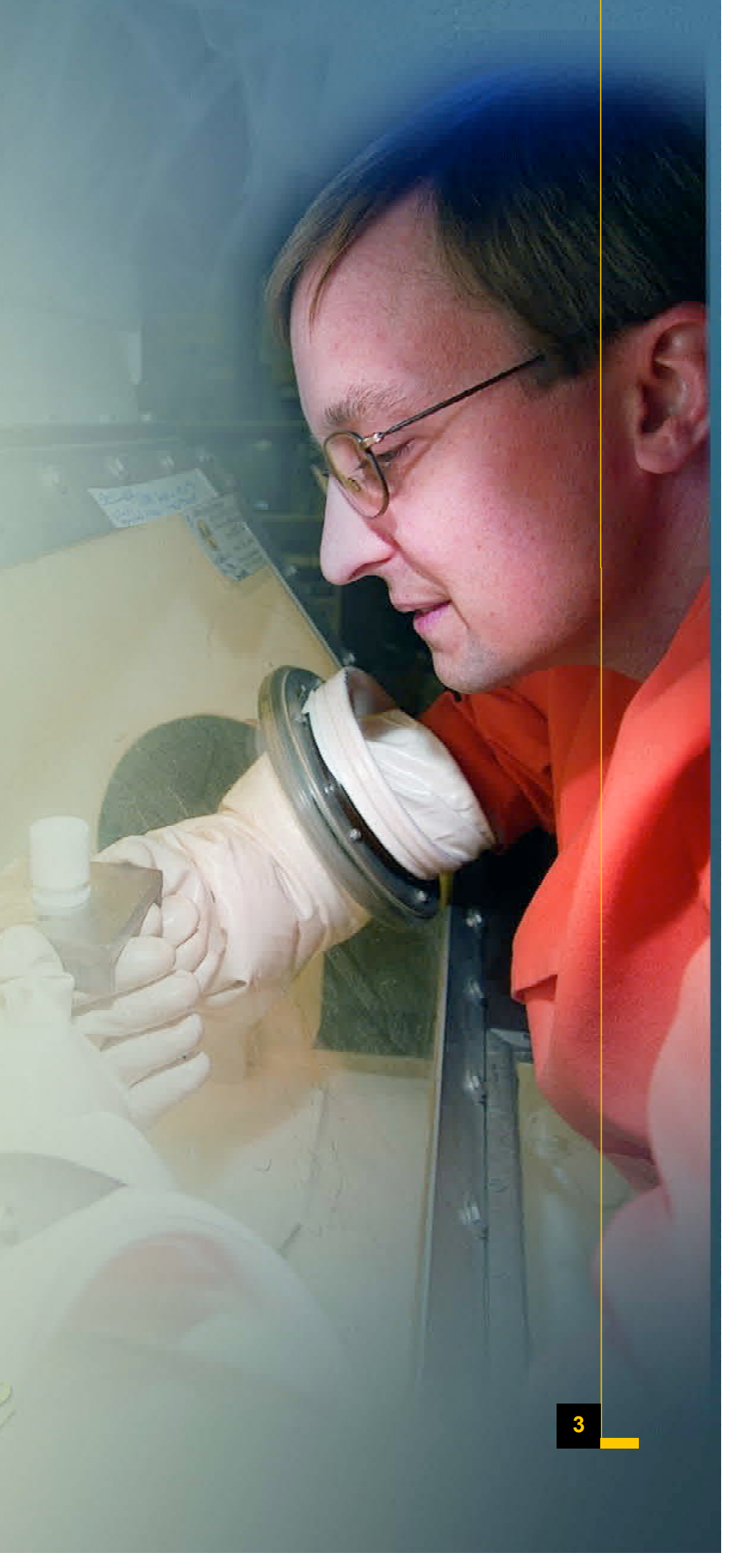


A new generation of experimental techniques enables us to probe materials under previously inaccessible conditions, chemical and physical phenomena. Our scientists also continue to improve the structural control in the synthesis of nanoscale sol-gel materials with desired composition and morphology.

\section{Applied Nuclear Science for Human Health and}

\section{Wional Security}

With tools such as the high-spatial-resolution NanoSIMS, our scientists are making dramatic advances in the science of isotope application to the investigation of interplanetary dust patticles, as well as to human-health. Additionally, nuclear science and isotopic techniques are proving critically important in defining and solving national and international problems related to the environment and global security. From isotope tracers from reprocessed nuclear fuel to groundwater contamination, the broad applications in science, health, and national security reinforce the importance of he enduring capability in nucle

\section{Dynamic Teams}

The Chemistry and Materials Science (CMS) Directorate

atepresent the culture of our directorate-discovery-class science and a dynamic organizational structure. We have six crosscutting scientific centers that are staffed by personnel from our three

divisions.

The three divisions in CMS are responsible for the line management and leadership of the scientific, technical, and administrative staff. Each division maintains a close relationshi program leaders to ensure an effective response to programmic

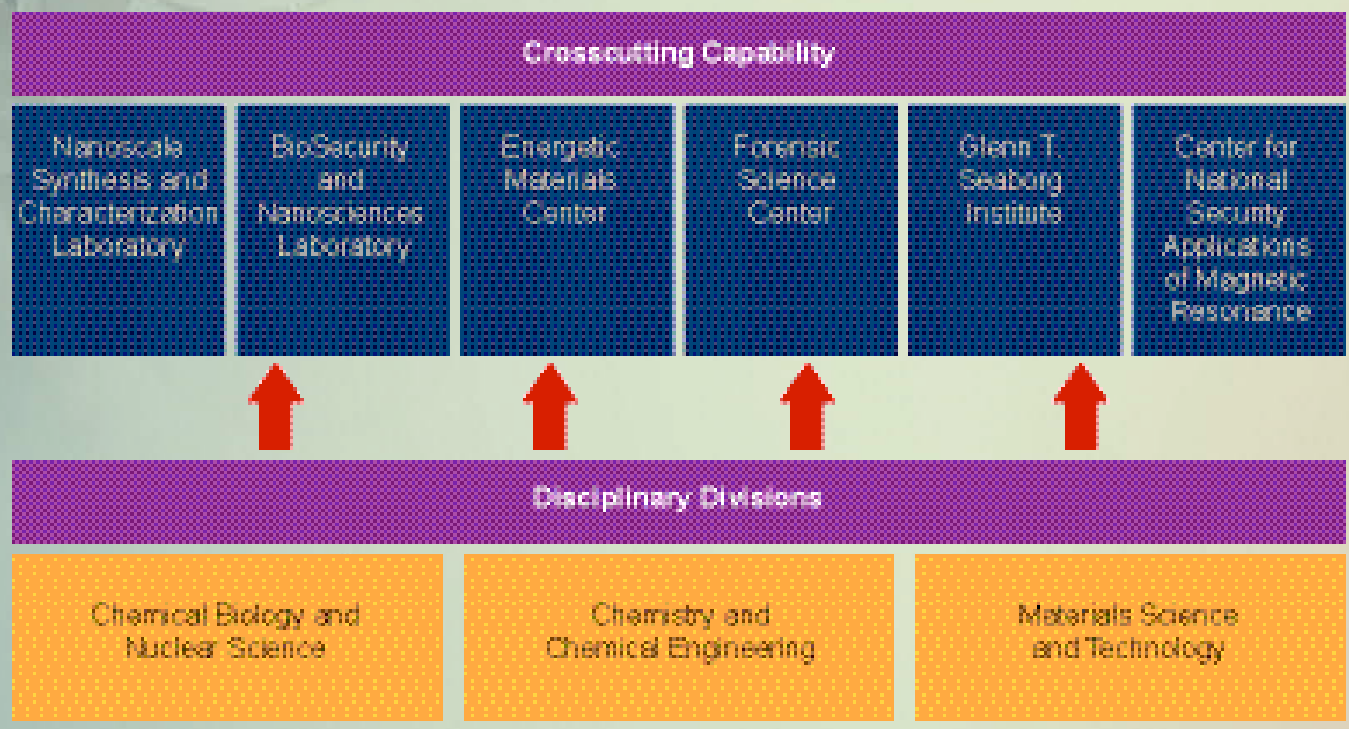

needs. Together, the divisions provide us with the flexibility and gility to respond to change and to merge our science successes to program milestones.

The centers provide specific research environments to bpport needs in nanoscale novel materials development: biosecurity and nanobiotechnology research; forensic science for chemical, nuclear, and biological counterterrorism; applied nuclear science and nuclear radiochemistry; research and development of high explosives, pyrotechnics and propellants

\section{Awards and Recognition} The staff who make the science happen at Lawrence
Livermore continue to receive recognition for their accomplishments from their peers through awards, honors, and ellowships from respected scientific societies. The award and recognition section of this report highlights the many chievements i

Financial and Operational Information

CMS is fully committed to the simultaneous excellence in both science and operations. In addition to the scientific an financial and operational data into a separate publication, Facts and Figures. This companion publication to the annual report intends to be a broad overview of budgetary, personnel, and other administrative information about Lawrence Livermore National Laboratory and our directorate. Facts and Figures is available for download from our websit.

\section{Looking Forward}

The future we envision is arriving, and more rapidly than unfolds and the Laboratory assumes increasing responsibilities in new national-security programs. CMS maintains an unambiguous role as both a partner and a technology leader for these responsibilities. More confident and committed than ever, we look forward to expanding frontiers of science and

continuing our legacy of partnership with the Laboratory and the worldwide scientific community, as we apply science
and technology to the important challenges of our time.

Sincerely,

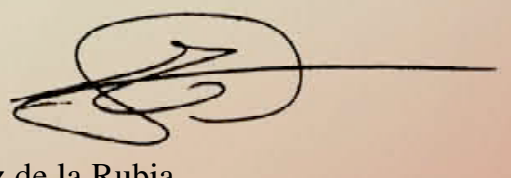

Tomás Díaz de la Rubia

Associate Director, Chemistry and Materials Science Lawrence Livermore National Laboratory 

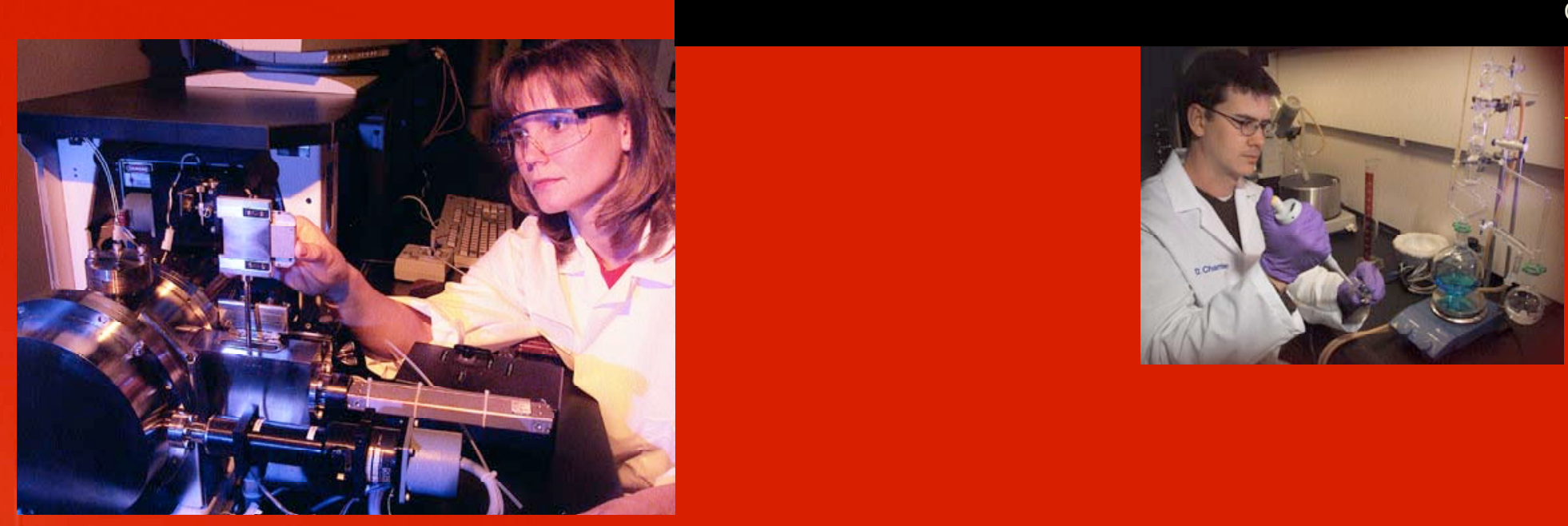

\section{Oresearch Oremes}

Science at the intersection

CHIEMISTIRY AIND MATERIALS SCIENCE Materials properties and performance

Chemistry under extreme conditions
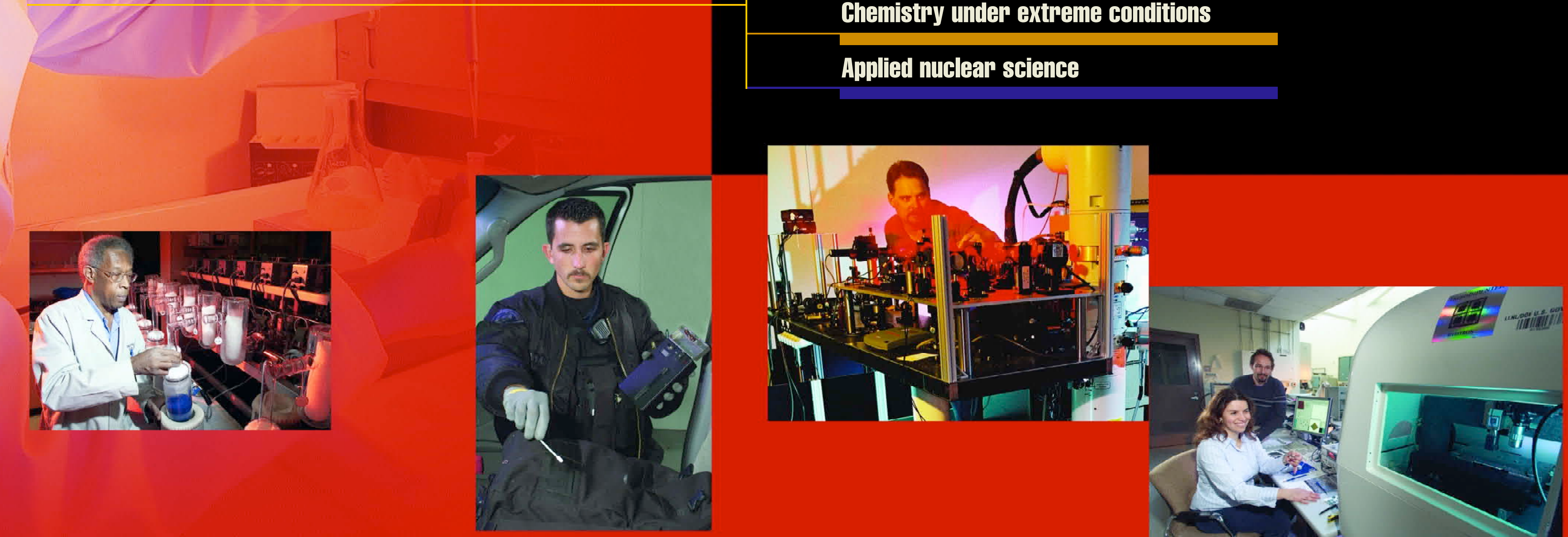
Anplied nuclear seience

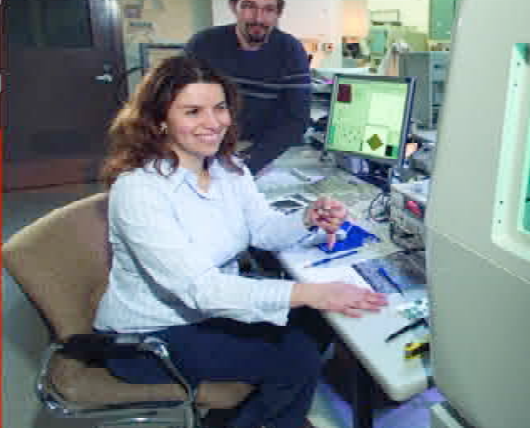




\section{Unraveling the Molecular Modulation of Kidney Stone Formation}

\begin{tabular}{|c|c|c|}
\hline $\begin{array}{l}\text { he primary constituent of kidney } \\
\text { stones in patients with urolithiasis } \\
\text { is calcium oxalate monohydrate (COM). } \\
\text { Normal urine contains compounds such } \\
\text { as citrate and proteins that inhibit } \\
\text { crystallization of COM (Figure 1). } \\
\text { Although these inhibitors provide } \\
\text { protection, their precise roles have not } \\
\text { been defined. Understanding the } \\
\text { molecular features and physiologic } \\
\text { effects of individual urinary proteins and } \\
\text { small inhibitory molecules is essential for } \\
\text { determining their roles in disease and } \\
\text { defining strategies for designing } \\
\text { therapeutic drugs. } \\
\text { We investigate the molecular } \\
\text { mechanisms of tissue mineralization and } \\
\text { pathogenesis by combining molecular } \\
\text { modeling and in situ scanning probe } \\
\text { microscopy. Using this method, we } \\
\text { examine the thermodynamic and kinetic } \\
\text { impacts of citrate and osteopontin on } \\
\text { modulating the growth of COM and } \\
\text { determine the stereochemical relationship } \\
\text { between the growth modifier and atomic } \\
\text { features on the crystal surface. }\end{array}$ & $\begin{array}{l}\text { Relevance to CMS Research Themes } \\
\text { Our work focuses on biomolecular } \\
\text { control of tissue mineralization and } \\
\text { pathogenesis. This field integrates } \\
\text { nkowledge from materials physics, } \\
\text { physical chemistry, biology, and medicine. } \\
\text { The work aligns with the CMS strategic } \\
\text { theme of science at the intersection of } \\
\text { chemistry, materials science, and biology. } \\
\text { Understanding how macromolecules } \\
\text { dictate the shape, size, and properties of } \\
\text { inorganic entities will strongly impact } \\
\text { efforts in biological, chemical, and } \\
\text { materials science and technology for } \\
\text { national-security applications. } \\
\text { Major Accomplishments in } 2004 \\
\text { We have found that COM grows on } \\
\text { atomic steps at dislocations, and citrate } \\
\text { and osteopontin each attack a different } \\
\text { face of COM crystals. Figure } 2 \text { illustrates } \\
\text { COM growth in a citrate-bearing solution. } \\
\text { Citrate predominately inhibits growth on } \\
\text { the (-101) face by forming a geometric } \\
\text { match between the carboxylic groups and } \\
\text { the calcium ions at the step edges. A }\end{array}$ & $\begin{array}{l}\text { triangular shape dislocation hillock } \\
\text { becomes quite rounded and disc-shaped. } \\
\text { Citrate has a much lesser effect on the } \\
\text { (010) face. } \\
\text { Molecular modeling calculations } \\
\text { show that the highest binding energy of } \\
\text { citrate bounds to steps on the (-101) face } \\
\text { is - } 170 \mathrm{~kJ} \cdot \mathrm{mol}-1 \text { and only }-102 \mathrm{~kJ}^{-1} \mathrm{~mol}^{-1} \\
\text { on the (010) face. Our experiental data, } \\
\text { along with theoretical calculations, } \\
\text { suggest that citrate modifies the shape and } \\
\text { inhibits the growth of the COM by } \\
\text { pinning-step motion, as indicated in } \\
\text { Figure } 3 \text {. In addition, the morphological } \\
\text { change in step structure is consistent with } \\
\text { that of the bulk crystal shape. } \\
\text { In contrast, osteopontin has the greatest } \\
\text { effect on the (010) face, where the step } \\
\text { height is similar to the size of the protein. } \\
\text { Osteopontin also inhibits the step kinetics } \\
\text { and changes its morphology. } \\
\text { These results suggest that, used } \\
\text { simultaneously, citrate and osteopontin } \\
\text { may work synergistically to inhibit overall } \\
\text { growth of COM. In addition to designing } \\
\text { drugs that reduce or eliminate kidney }\end{array}$ \\
\hline
\end{tabular}
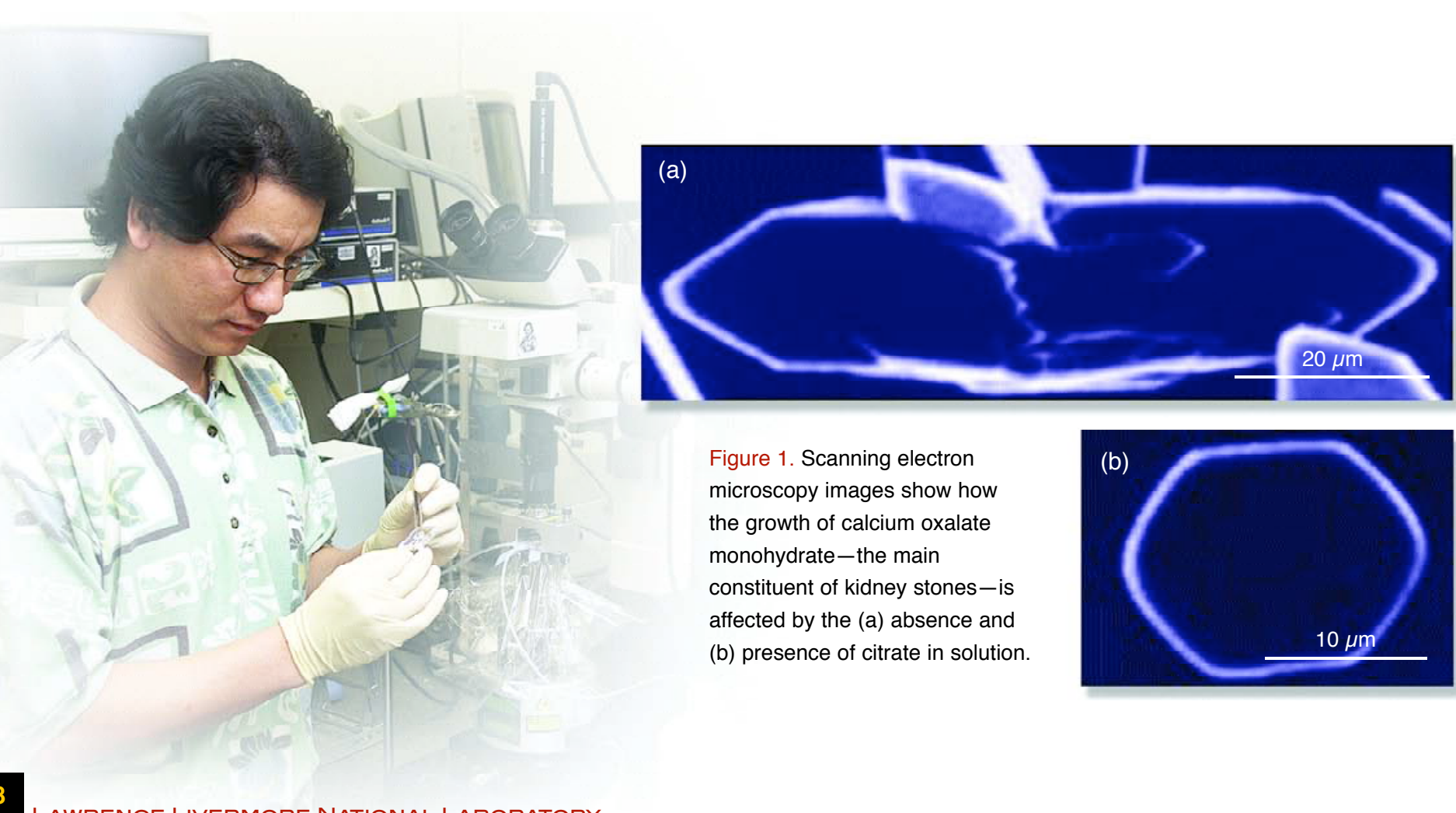

Figure 1. Scanning electron microscopy images show how
the growth of calcium oxalate monohydrate-the main constituent of kidney stones - is affected by the (a) absence and (b) presence of citrate in soln

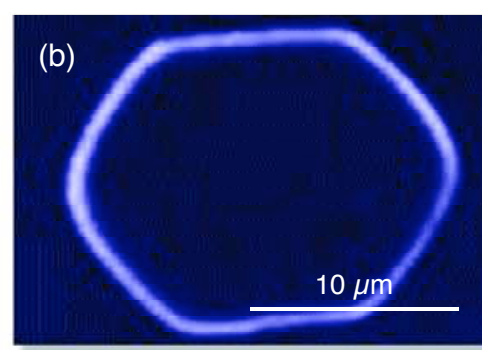

For more information contact

ROGER QIU (925) 422-1636, qiu2@llnl.gov

stones, these modifiers are effective at provide materials scientists with strategies for growing designer crystals. The resuls
also demonstrate the capability of also demonstrate the capability of combining molecular imaging and
modeling tools for understanding eve in disease.

Scientific Impact

xalate crystallization. In addition changing the shape of crystals and may underlying aberrant crystallizatio These results, which were recently featured as a cover story in the Proceedings of the National Academy of Sciences, showed for the first time the molecular mechanism of therapeutic

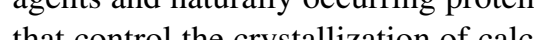
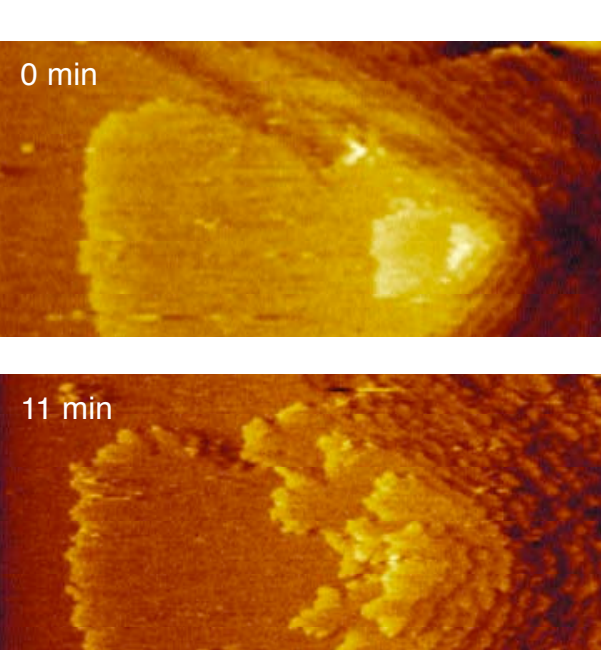
作 o inhibit kidney stone growth.

Related Publications S.R. Qiu, et al., "Molecular Modulation of Calcium Proc. Natt. Acad. Sci USA 101, 1811-1815 (2004). J.J. De Yoreo and P.M. Dove, "Shaping Crystals with Biomolecules," Science 306, 1301-1302 (2004). P.M. Dove, J.J. De Yoreo, and S. Weiner, Eds., Reviews in Mineralogy and Geochemistry, Volume 54 Blominerallzation (Mineralogical Sociely o I Amenta

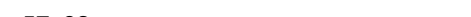
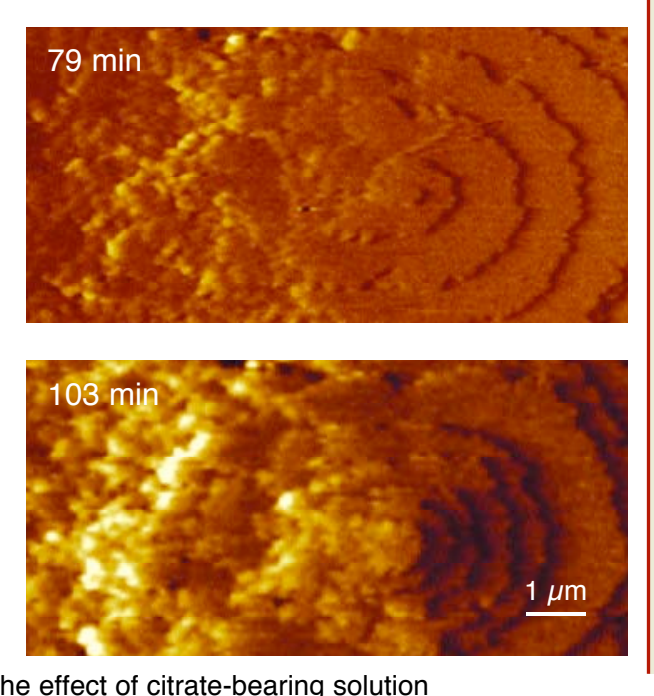

NEW FRONTIERS

By combining theoretical calculation with crystal growth theory, we will define the mechanism by which small thermodynamis and kinetios of the COM growth and shape modification. We will also investigate the interaction between the COM crystal and synthetic peptides that mimic the acidic segments of osteopontin and are believed to be responsible for the inhibitory function. Understanding the mechanisms by which peptides interact with the COM acsials reveals detalls of various amino control the crystallization of kidney stones in humans. Knowledge about the molecular modulation of kidney stones can be extended to studies of other biogenic materials in humans, such as the mineral phases of calcium

posphate- the main inorganic
component in human teeth and bones. We expect that this knowledge will lead to the development of new drugs to also help researchers design strategies to remove heavy metals from groundwater.

Figure 2. Atomic force microscopy images show the

Figure 3. Molecular modeling calculations show

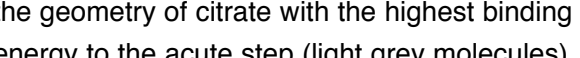
on the $(-101)$ face. The green lines represent the chemical bonds between the citrate molecules and calcium ions, and the bonds between the hydroxyl groups and the oxalate ions. Other ions represented are $\mathrm{Ca}^{2+}$ (blue), $\mathrm{O}$ (red), C (dark grey), and $\mathrm{H}$ (off-white),

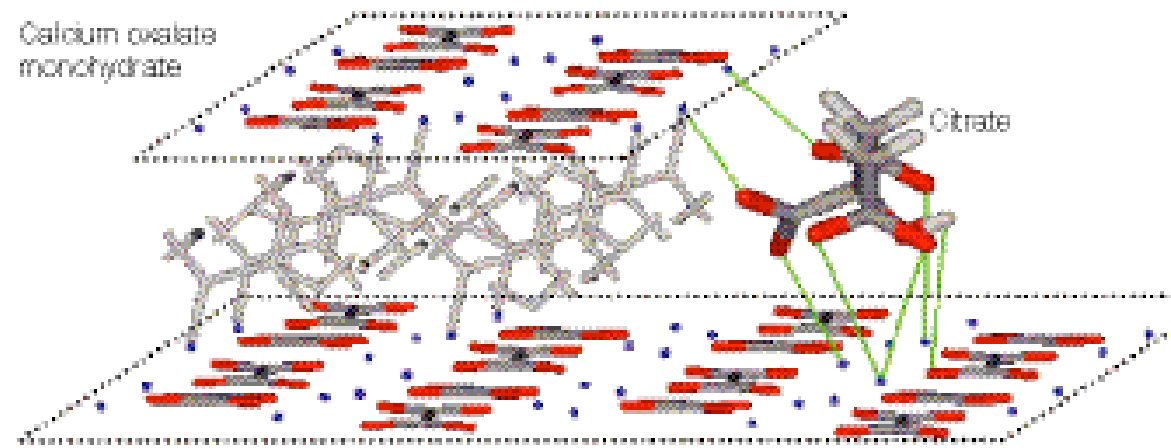




\section{Smart Membranes for Bio-Organism Detection}

7 dentifying biological organisms that may have been engineered for the purpose of a biological attack is critical
for national security. To address this need we are coupling semiconductor
nanotechnology with surface chemistry. nanotechnology with surface We have synthesized arrays of membranes that can selectively capture and immobilize an organism ranging in size from spores (many micrometers) to viruses (tens of nanometers), as

illustrated in Figure 1

Creating channels in the membrane with colted position, length, and diameter to a few ins of fion many micrometers major challenge. Furthemore in cofised geometries such as nanochannels, rolling and characterizing the

significant technological breakthroughs. To identify bio-organisms, we are building a particle-characterization de based on a Coulter counter, with a fabricated pore that mimics an device, it generates an inic through the blockade event The amplicule, durto and shape of the electrical signal provide information on size, shape, and chemical composition of the organism (Figure 2). Relevance to CMS Research Themes Understanding the interface between a inorganic platform and organic ligands is aligned win the part of the CMS strategic plan that is dedicalod to systens Livermore's national-security mission building a detection platform that will

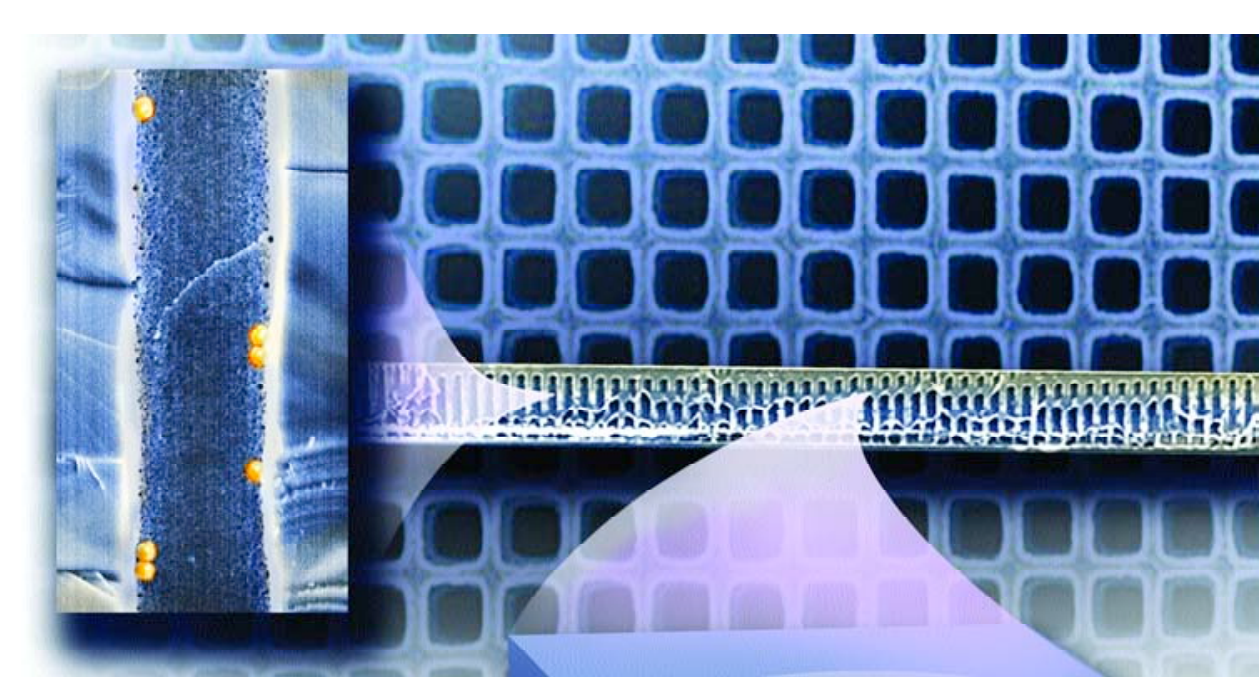

Figure 1. A functionalized silicon membrane is shown in the background. A close-up (leff) and cross section (center) selectively captures simulated bio-organisms, represented orang. generate a profile of characteristics such as size, shape, and chemical functionality of unknown biological organisms. Once characterized, the electrical signatures of 作

Major Accomplishments in 2004 Our group demonstrated the first success silicon membranes and showed their ability to selectively capture micro-organisms. We produced periodic arrays of functionalized pores with diameters down to $30 \mathrm{~nm}$ and aspect ratios (opening vs depth) up to 250 which had never been achieved before. To flurescer The membranes, functionalized with a particular antigen, selectively captured the matching antibody-coated beads while allowing the rest of the beads to flow

through the membranes' pores

We also demonstrated the utility of our devices for cell encapsulation application range. In such applications, the main challenge is to fabricate a porous capsule hat allows the diffusion of a cell nutrien such as glucose, while blocking the immunoglobulin, which may trigger

In each application, we used a focused ion beam to machine single apertures down through these devices was recorded with through these devices was rec functionalization, demonstrating the open nature of the apertures before and after achieved localized oxidation deposition at the entrance of the membrane aperture, which we characterized by energy dispersive $\mathrm{x}$-ray spectroscopy and atomic force microscope spectroscopy.

For more information contac

SONIA E. LÉTANT (925) 423-9885, letant1 @ llnl.go

We are now studying the dynamics of DNA probes as they flow through functionalized apertures (Figure 3) in order to demonstrate the selectivity of our Coulter counter. The next step will be demonstrate selective bio-organism

Scientific Impact single-pore technology is heading towar ran blood cells a the ocs as large as

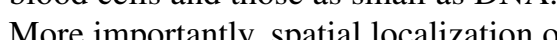

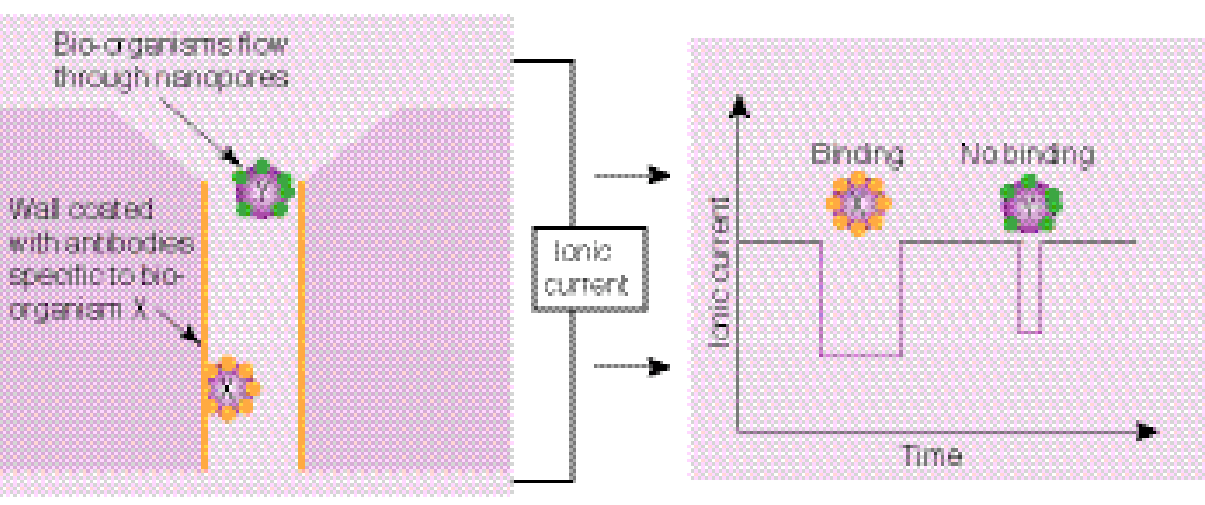

Figure 2. The scheme of operation shows a bio-organism traveling through a functionalized Coulter counter. An ionic current drives the bio-organism through an aperture. The amplitude of the blockade the organism's outer of coat proteins and the functionalized aperture wall

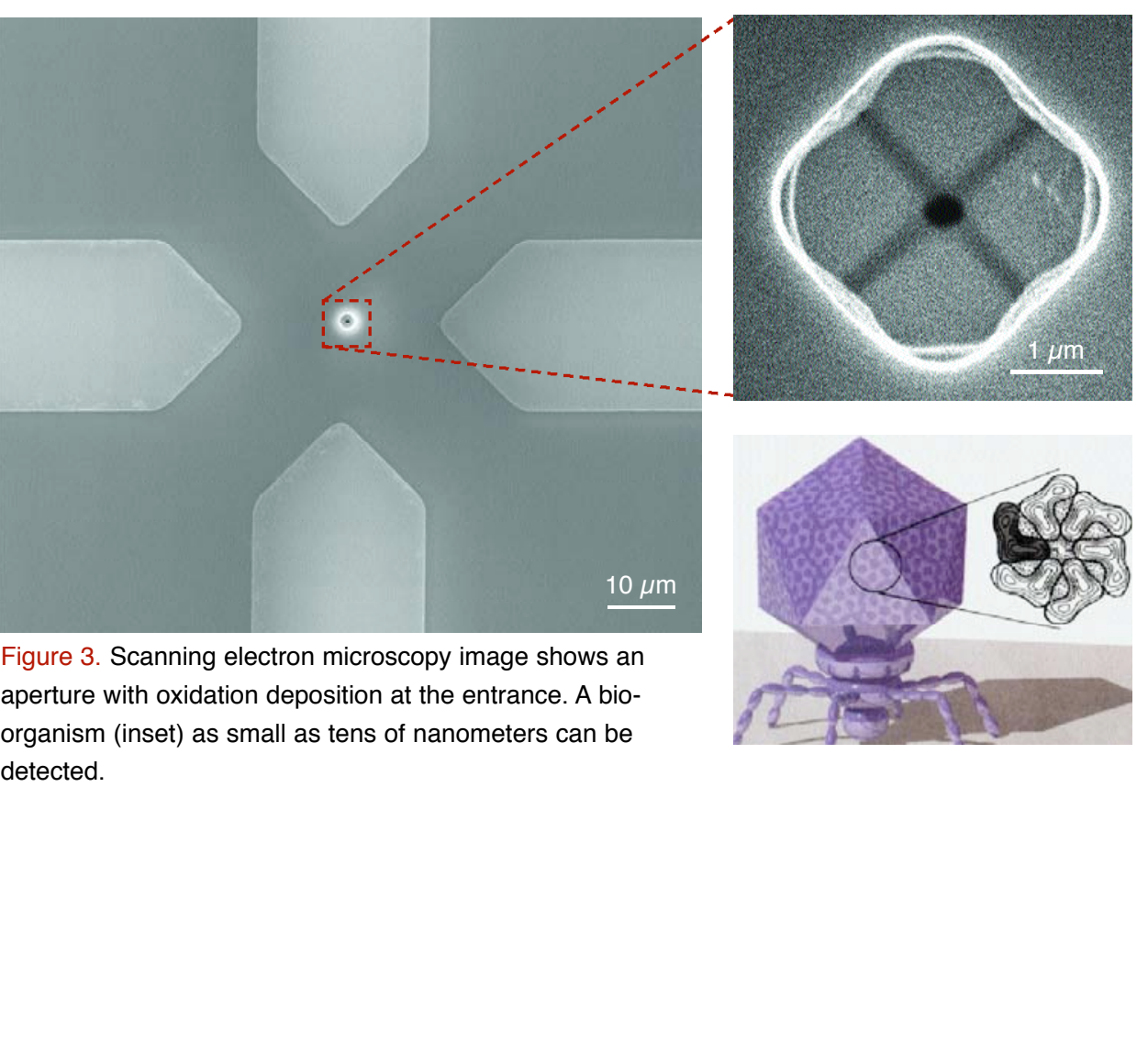

the ligands on artificial pores has not achieved. Our group is closing the gap by machining single apertures to the - 0 -nm range to study virus and beam-assisted oxide growth around the Dertures, followed by self assembled surface chemistry, to demonstrate localization of the ligands at the aperture opening. This unique combination of controlled aperture geometry and surface chemistry should lead to the first organisms.

Related Publication E. Létant, et all, "Functionalized Silicon Membranes

E. Létant, et al., "Nanochannel Arrays on Silicon Pattorms by Electrochemistry," Nano Letters $4(9)$ $1705(2004)$

\section{NEW FRONTIERS}

When this new class of detection platorns is developed, sclentssts wit analyze bio-organisms in real time. These engineered membranes are extremely versatile and could be adapted to recognize the fingerprints (size and membrane proteins) of any bio-organism of interest. To help achieve that goal, we will add in situ dection capabil wes to our selective such an integrated device include: counter-terrorism, human health, and nvironmental monitoring. A challenge will be to further control single-aperture functionalization, tingle chemical postioned at the entrance of a and to derture. Such a plattorm would and to the sequencing of a sinecules molecule of DNA. 


\section{Molecular Transport in Carbon Nanotubes}

7 mproving our capability to detect and monitor chemical and biochemical mission. Our group takes a molecular-leve approach for materials transport and uses to develop innovative nanostructures that can be used for biological agent detection desalination and molecular separation. Discovered in 1991, carbon nanotubes are extremely small, hollow cylinder structures that are endowed with exceptional conductivity and strength. Particularly, one-dimensional nanowires and nanotubes exhibit unusual physical properties, due to their reduced dimensionality and enhanced surfacemolecular wire templates and utilize the unique geometry, structure, and electrica properties to create novel functionality in two project areas. First, we are building nanodevice that can detect pore-forming bacterial toxins. In another project, we are building a synthetic membrane that would utilize the inner diameter of the nanotube for mass transport and separation.

Relevance to CMS Research Themes This work advances system integratio strategies by developing sensing and
surveillance components for a broad spectrum of chemical and biochemical applications. For example, onepolyelectrolyte polymers, we can control the nanotube surface functionality and ads (t) support and strength for the lipid bilayers

surface of a semiconductor chip and will formation of one-dimensional lipid enable the use of low-cost electrical detection to interrogate the sensors. The tight integration of biomimetic a inorganic components in such a structure can control access of the analyte molecular precision. The technology can be applied to other relevant areas,

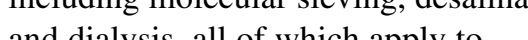

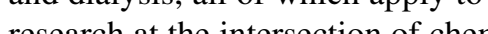
materials science, and biology.

Major Accomplishments in 2004 The phospholipids, or lipids, in cell membranes are arranged as a bilayer (two molecules thick). We have successfully fabricated and characterized one-
dimensional lipid bilayers, which dimensional lipid bilayers, which
represent a new class of nanostructures biomimetic membranes (synthetic membranes that mimic biological ones, as illustrated in Figure 1. By modifying bilayers in which a lipid bilayer nanostructures to form an insulating jacket. By applying the fluorescencewe have confirmed that the lipid molecules in these nanostructures are mobile (Figure 2).

In our second project, we have developed a synthetic membrane by usin carbon nanotubes with diameters of Graphite's inherently smooth suf enables a high flux of the permeate molecules. Recent simulations by our Health suggest that water exhibits unique effects upon one-dimensional confineme not seen in the bulk, and our aim is to produce a platform for investigation of Uning.

Using standard microfabrication lechniques, we have produced patterned (Figure 3) with outer diameters of 20-50 nm and inner diameters of ensures the ultimate sensitivity of the molecules to the chip components wit recovery-after-photobleaching techniques, collaborators at the National Institutes of

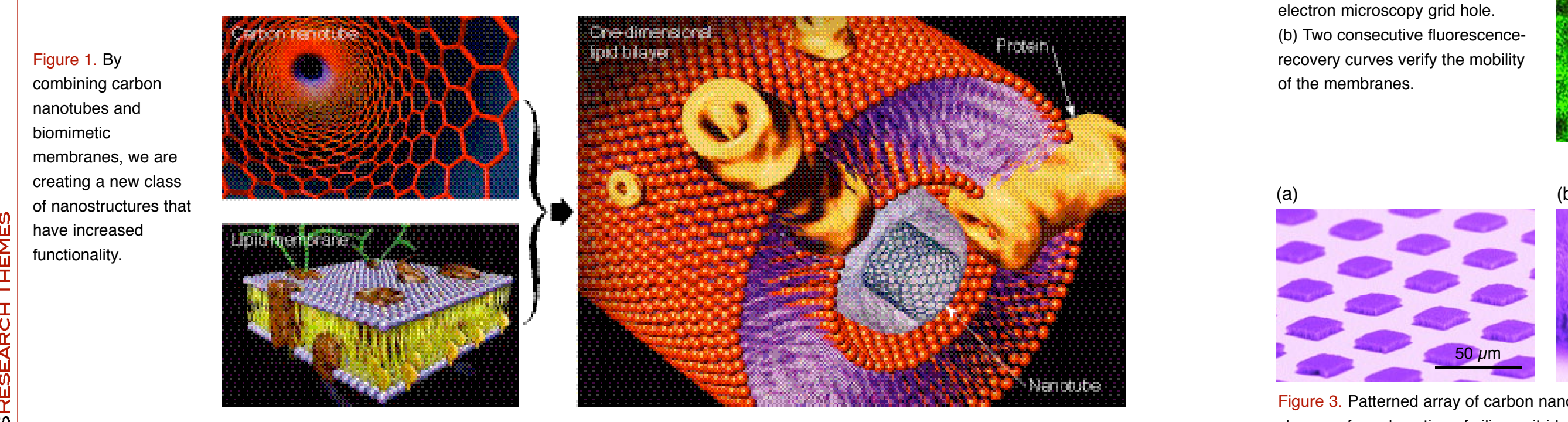

6-12 $\mathrm{nm}$. The arrays are coated with a Porosimetry measurements have confirmed that the membrane is void-free with pores corresponding to the in nanotubes. characterize the transport characteristics of electrolytes through this membrane for Efforts are also under way to produce the single-wall nanotube version of this membrane (pores sizes from 0.6-2 nm) Which would enable highly selective closer to observation as well as bring un effects in water.

Scientific Impact of unique nanostructures that tightly
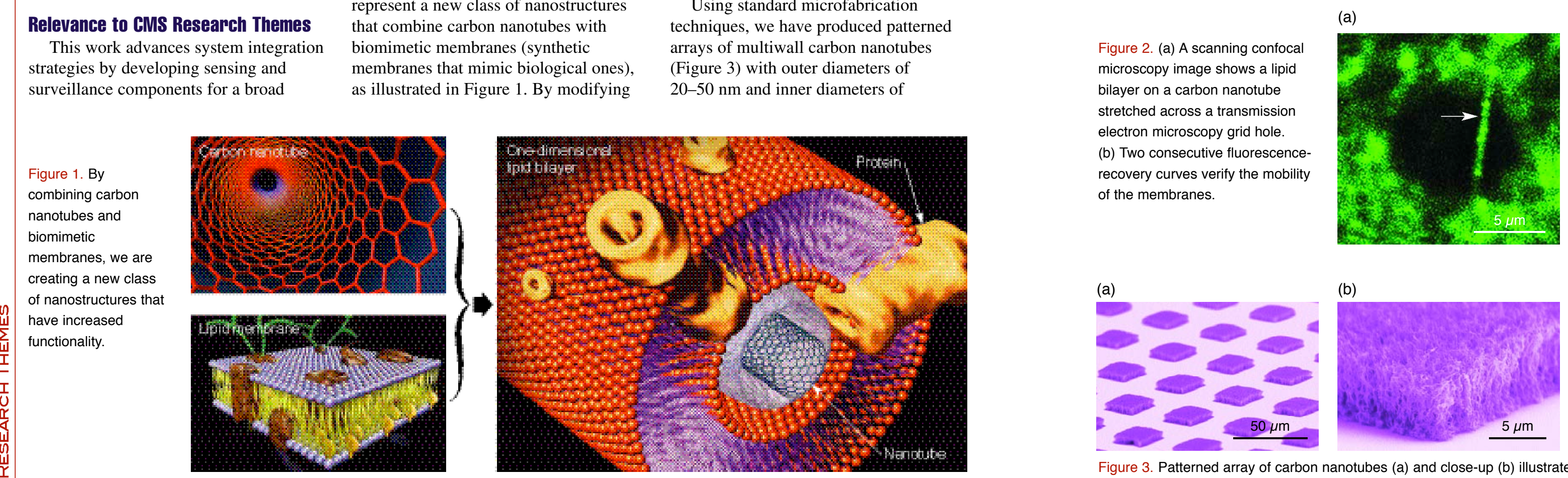
A.B. Anyunin, A al., Functional One-Dimensional conformal silicon nitride matrix to fill the gaps between the tubes and to produce robust, freestanding membrane.

Experiments are under way to applications in desalination and dialysis.

Our work on the one-dimensional lipid bilayers is one of the first demonstration

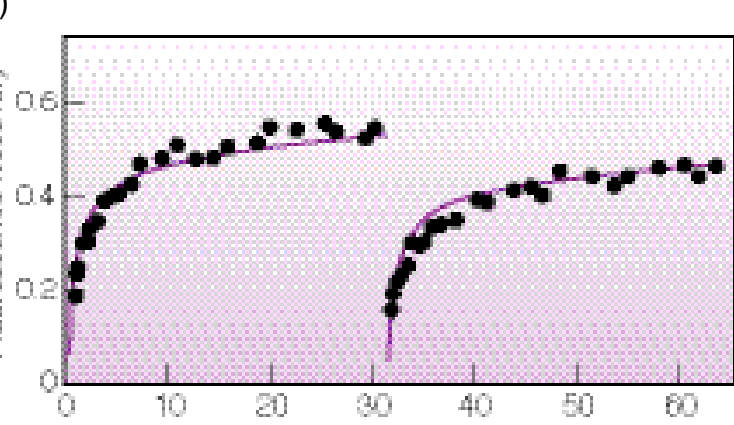

inorganic nanostructures. This integration allows for the efficient transformation of electrical responses which results in many new opportunities to advance our neves allow us to test the predictions from simulations that show unique effects of confinement in bulk water.

Related Publication Chem. Soc, in press.

A.B. Artyukhin, et al,, "Layer-by-Layer Electrostatc Self-Assembly of Polyelectrolyte Nanoshells on 20(4), 1442-1448 (2004).

J. Hott, et al, "Fabrication of a Carbon NanotubeEmbedded Silicon Nitride Membrane for Studies of Nanometer-Scale Mass Transport," Nano Letters 4(14) Nanometer-Scale stady of molecular transport and to desig

\section{NEW FRONTIERS}

Carbon nanotube membranes provide a foundation for new types of materials and structures that can be used in many applications, including water desalf aplion and ulla-lough properties. Our studies will make a significant contribution to fundamenta studies of molecular transport in confined nanostructures and to the nascent field of nanofluidics. As we continue our work on fabricating structures based on one-dimensional bilayers, we will incorporate them in detection devices that utilize the proteins. We will also investigate Itternative uses for this technology for Finally, the one-dimensional bilayer provides unique capabilities for systems of reduced geometry and molecular-scale curvature.

igure 3. Patterned array of carbon nanotubes (a) and close-up (b) illustrate the typical high density, in excess of $10^{10} \mathrm{~cm}^{-2}$ (areal). Mirrograph membrane (d), with pore sizes representative of the nanotube outer diameters. studying molecular transport in 


\section{Novel Approach for Identifying Bacterial Toxin Inhibitors}

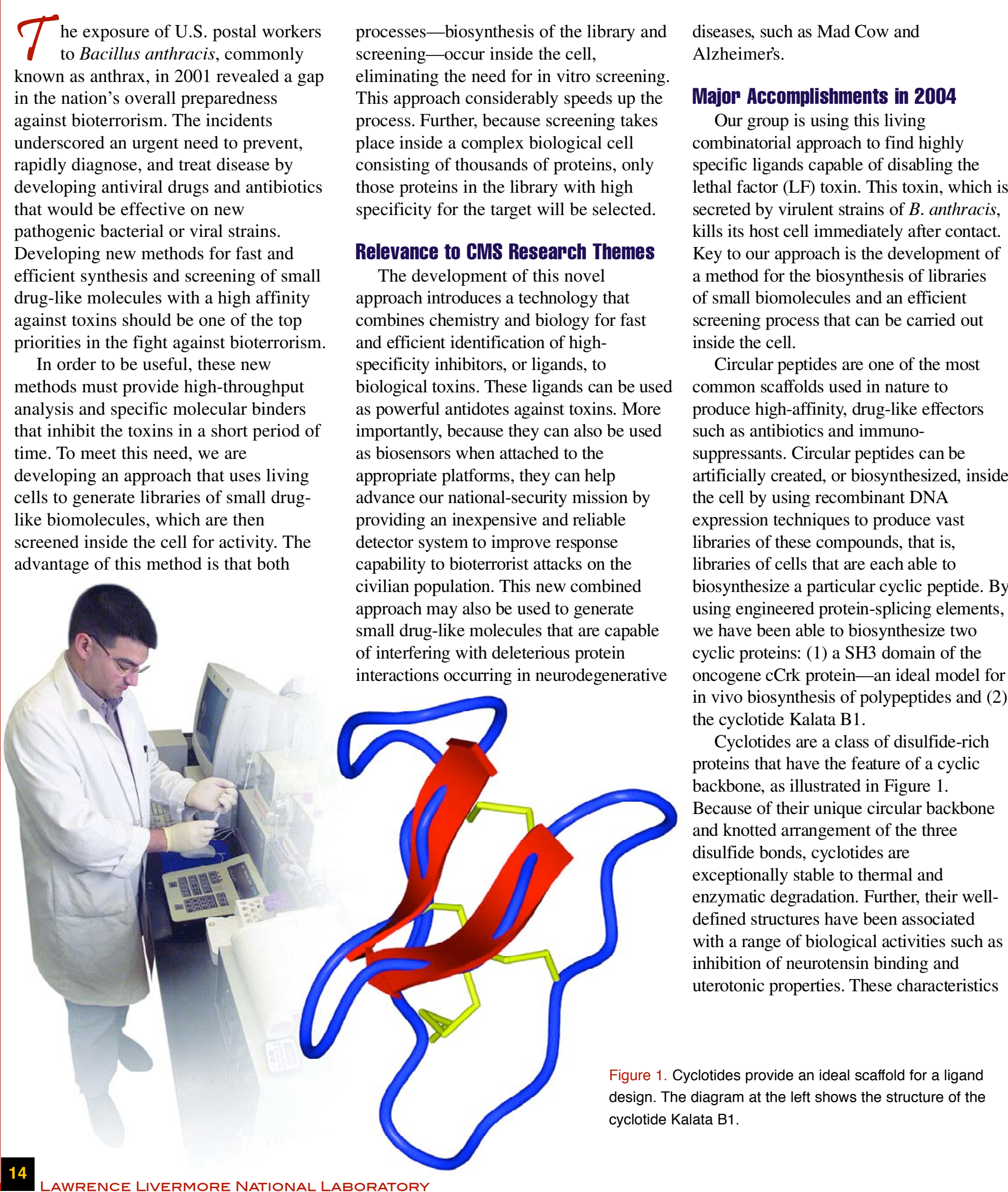

For more information contact

JULIO A. CAMARERO (925) 422-6807, camarero1@llnl.gov
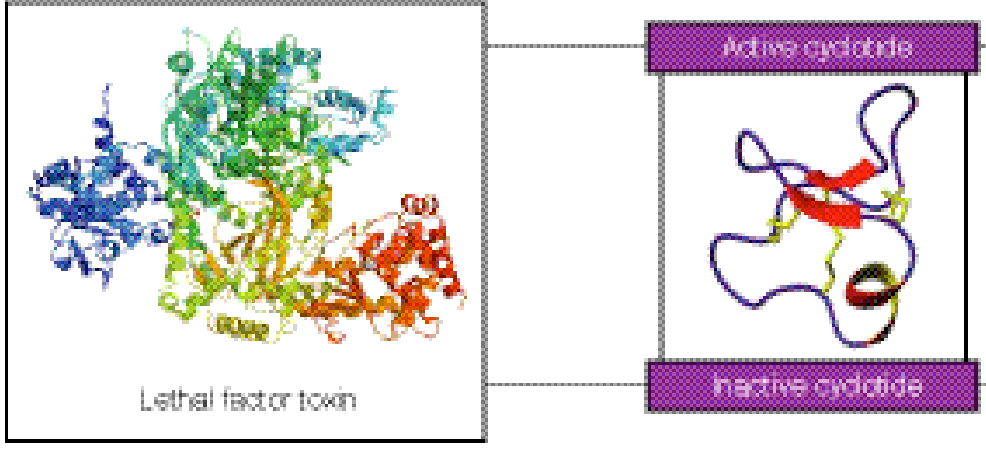

Town auteratate flanked th

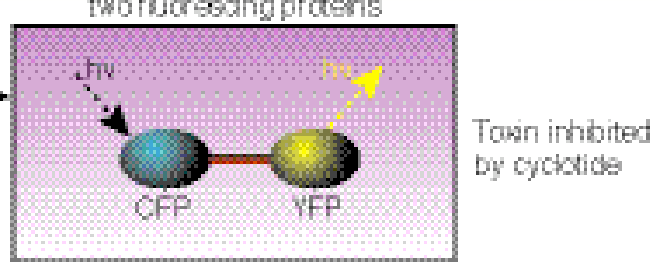

Figure 2. We have developed an in vivo appionct B. anthracis. The chameleon reporter directly senses the toxin activity and reports through a fluorescence change which cells express active inhibitors. In a cell with an active cyclic peptide inhibitor, we can measure the emission of the yellow fluorescent protein (YFP) fluorophore as
the cyan fluorescent protein (CFP) fluorophore is excited.

make cyclotides ideal candidates as molecular scaffolds for new ligand/drug design and discovery. We use fluorescent proteins as "Chameleon reporters" in vivo during the screening process to find which cyclotides inhibit the LF toxin produced by $B$. anthracis (Fig toxin produced by $B$. anthracis (Figure 2). The reporter monitors nolecular interactions and ( that cleaves specific proteins critical for the survival of the targeted cell. The screening reporter uses the catalytic activity of the protease enzyme in combination with a fluorogenic substrate, which is composed of a short substrate the protease and is flanked by two fluorescent protens - cyan and yellow. Cyan fluserest engineered mutants of the naturally occurring green fluoresent protein are designed to form a fluorescence resonance energy transfer (FRET) pair. When the protease and the fluorogenic substrate are expressed in a cell that has biosynthesized an active cyclic peptide inhibitor, the protease is unable to cleave to oberve the FRET by Messions emission of the YFP fluorophore as the CFP fluorophore is excited. In contrast, we observe no FRET in a cell that has no biosynthesized an active cyclic peptide nzymatic assay in combination with fluorescence-activated cell sorter technology, we are able to separate the cells capable of producing selective intibitors. Also, DNA sequencing or the identify the active component of the library that can be later used in detec or disease treatment.

\section{Scientific Impact}

Our initial focus has been to produce high-affinity ligands that can disable bacterial and other biological toxins. find sll dug like can easily be used to find snall drug-like molecules capable

be used to find molecules that mad can the destructive mechanisms involved in cancer and neurodegenerative diseases such as Mad Cow and Alzheimer's.

Related Publication J.A. Camarero and R. Kimura, "Expressed Protein Ligation: A New Tool tor he Blosynhinesis or Cy
A. Camarero, et al. "Biosynthesis of the Cyclotide Kalata B1 Using Protein Splicing Protein Sci. 13(1). 139 (2004).

J.A. Camarero, et all, "Peptide Chemical Ligation Inside Living Cells: In Vivo Generation of Circular Protein Domain," Bioorg. Med. Chem. Lett. 9, 2479-2484 (2001).

\section{NEW FRONTIERS}

We will develop a new method for the in vivo screening of biomolecular which only the cells that produce ligands capable of disabling the target protein will be able to survive. This method will simplify the selection process by system works. The ability to system works. The ability to
biosynthesize cyclic peptides using protein-splicing technology has important implications for drugdevelopment efforts. The capability to screen for biochemical events in an environment as complex as the cell's interior will provide valuable information. or example, peptide-based libraries are already showing to be effective in producing drug candidates in bacter
as well as mammalian systems. 


\section{Synthesis and Characterization of Nanoporous Metal Foam}

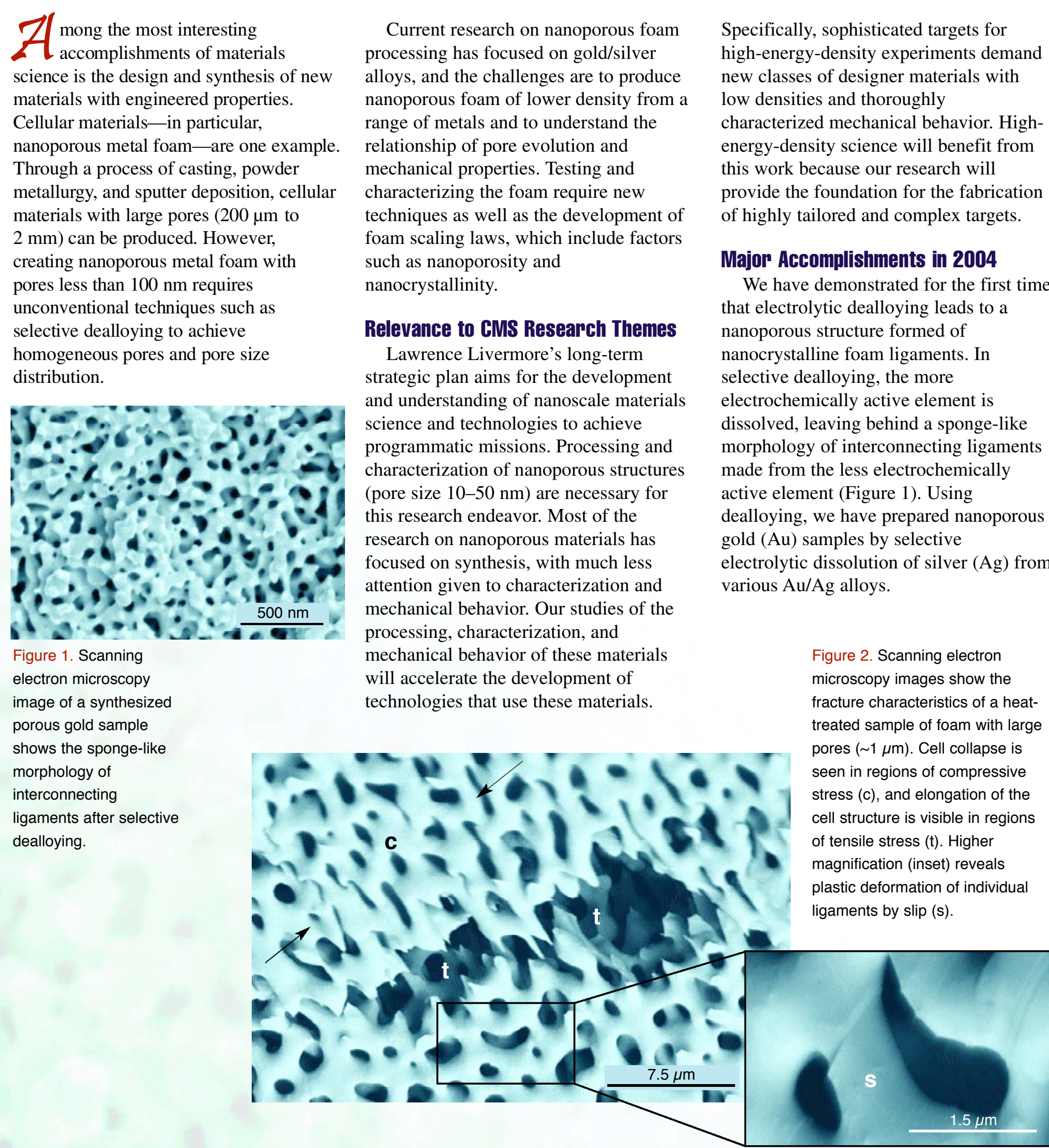

For more information contac

ANDREA HODGE (925) 424-3715, hodge4@1lnl.gov

Contrary to our results, previous research on free-corrosion dealloying (electroless) indicated that the dealloying process would not change the crystal grain structure to nanocrystalline. Thi discrepancy could suggest that produces a higher supersaturation of datoms, which in turn should incresse the nucleation rate. The actual evolving morphology should be strongly influenced by the mobility of vacancies and adatoms and, in particular, by the presence of nucleation sites and thus the developmen of a nanocrystalline structure.

Even though nanoporous metals have recently attracted considerable interestfueled by potential sensor and actuator their mechanical properties. To elucide the yield strength of nanocrystalline gold we have conducted both compression and tensile studies with nanoindentation and bending tests. Based on foam scaling laws, our results suggest that the nanoporous nanocrystalline gold is a high-yield, hig strength material that approaches trinsic yield strength of gold In ts also important to understand how materials would fail. We have deduced that the failure of a few ligaments trigges brittle fracture of the crystal lattice network. Interestingly, the failure mechanism of the ligaments seems to change with the ligament diameters. Ou microscopic characterization of fracture surfaces has shown that in nanoporo 100 $\mathrm{nm}$, the ligam dits $\mathrm{f}$. by flow and necking (elongation of

filaments). On the other hand, failure by slip (atomic plane movement) was observed for ligaments with a diameter $\sim 1000 \mathrm{~nm}$. Figure 2 shows the fracture characteristics of a heat-treated sample. The heat treatment increases the ligam diameter from $\sim 100 \mathrm{n}$ time that nanoporous foam synthesize less than $10 \mathrm{~nm}$ and hardness values of

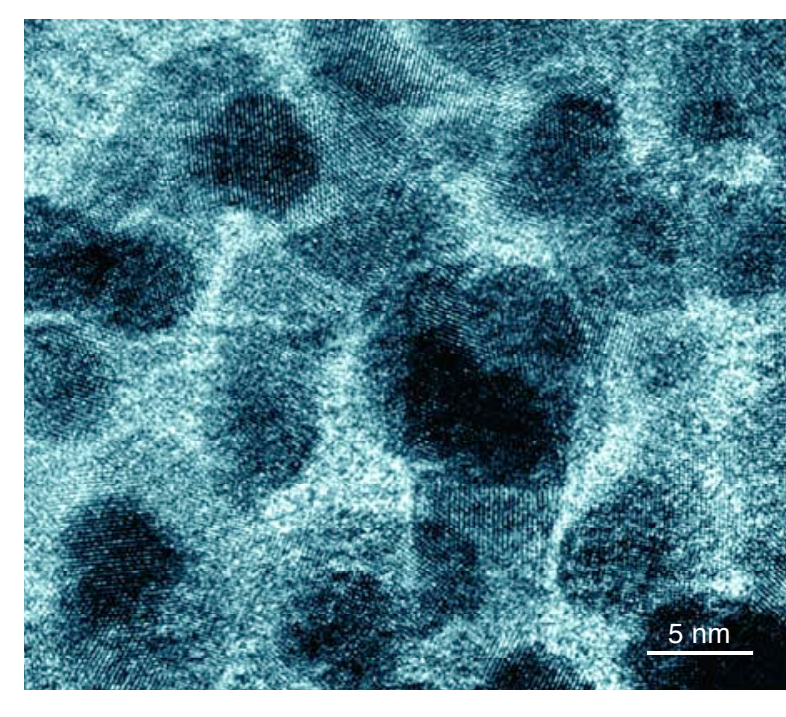
ransmission electron microscopy image shows of nanocrystalline
monolithic gold with grain monolithic gold with grain

Scientific Impact

Prior to our discovery, the general the field was that there was and therefore the nanoporous foam was composed of single-crystal

ligaments. We have shown for the first by dealloying (free-corrosion or electrochemically driven) is compos We also intionts. dealloying/compaction process to nanocrystalline monolithic gold (Figure 3). The compacted nanocrystalline gold exhibits an average grain size of up to 4.5 times higher than the values obtained from polycrystalline gold. Thi two-step process presents an alternative nanocrystalline metals.

Related Publications

Biener, et al., "Nanopoporous Au - a High Yield Strength Material," J. Appl. Phys. 97,024301 (2005)

A.M. Hodge, et al, "Monolititic Nanocrystalline Au Fabricated by the Compaction of Nanosscale Foan: J. Mater. Res. 20, $554(2005)$
NEW FRONTIERS

Nanoporous gold foam is an that can be synthesized with engineered properties.
Creating the foam with pores less than $100 \mathrm{~nm}$ through dealloying open the possibility for many applications. For example, nanoporous gold foam could be used for research on surface Chanced Raman spectroscopy. help to enhance Raman scattering, more recently, the use of gold nanoshells has been shown to significantly further increase the Raman scattering. The study of nanoporous foam as Raman enhancers and their comparison to nanoshells presents an interesting and technologically challenging project hat could impact Nanoporous fal and scientific fields. potentially used as actuators. Rec a nanoporous platinum material demonstrated the basis for the firs metal actuator, and many other applications are expected to arise for metallic actuators as new research becomes available. 


\section{Unraveling the Mysteries of Dislocation Formation}

$\int_{\text {ince the invention of the }}$ transmission electron microscope (TEM), materials scientists and physic
have investigated the structure of have investigated the structure of
dislocation networks in crystalline dislocation networks in crystalline
materials to understand the origins of their materials to understand the
unique mechanical strength. unique mechanical strength.
Dislocations-the carriers of plastic deformation in crystalline materialsincrease in density by several orders of magnitude during the deformation process. The interaction among dislocations and the topological rearrangements that occur in dislocation collisions are believed to responsible for strain hardening, a remarkable property of metals in which a material's strength increases Two colliding distoca pertions may bounded by two nodes. The behavior of dislocation nodes (where three or more dislocation lines connect) and the constraints they place on the motion their lines are believed to strongly influence the heterogeneous microstructures that develop with large

deformations. Unfortunately, the dislocations in these microstructures become so severely entangled that they can no longer be individually
distinguished by TEM. With the distinguished by TEM. With the development of the Parallel Dislocation silements of these highly entangl dislocation microstructures can now be investigated, providing a means for in computational microscopy.

Relevance to CMS Research Themes Lawrence Livermore's stockpile stewardship mission requires an understanding of the thermo-mechanical behavior of metals hat are subject to extring dislocations behave in ways the experiments are unable to assess and the existing theory is unable to foresee. ParaDiS allows us to investigate these behaviors in microscopic detail to understand how the dislocation microstructure forms and how it affects the mechanical behavior of the material.

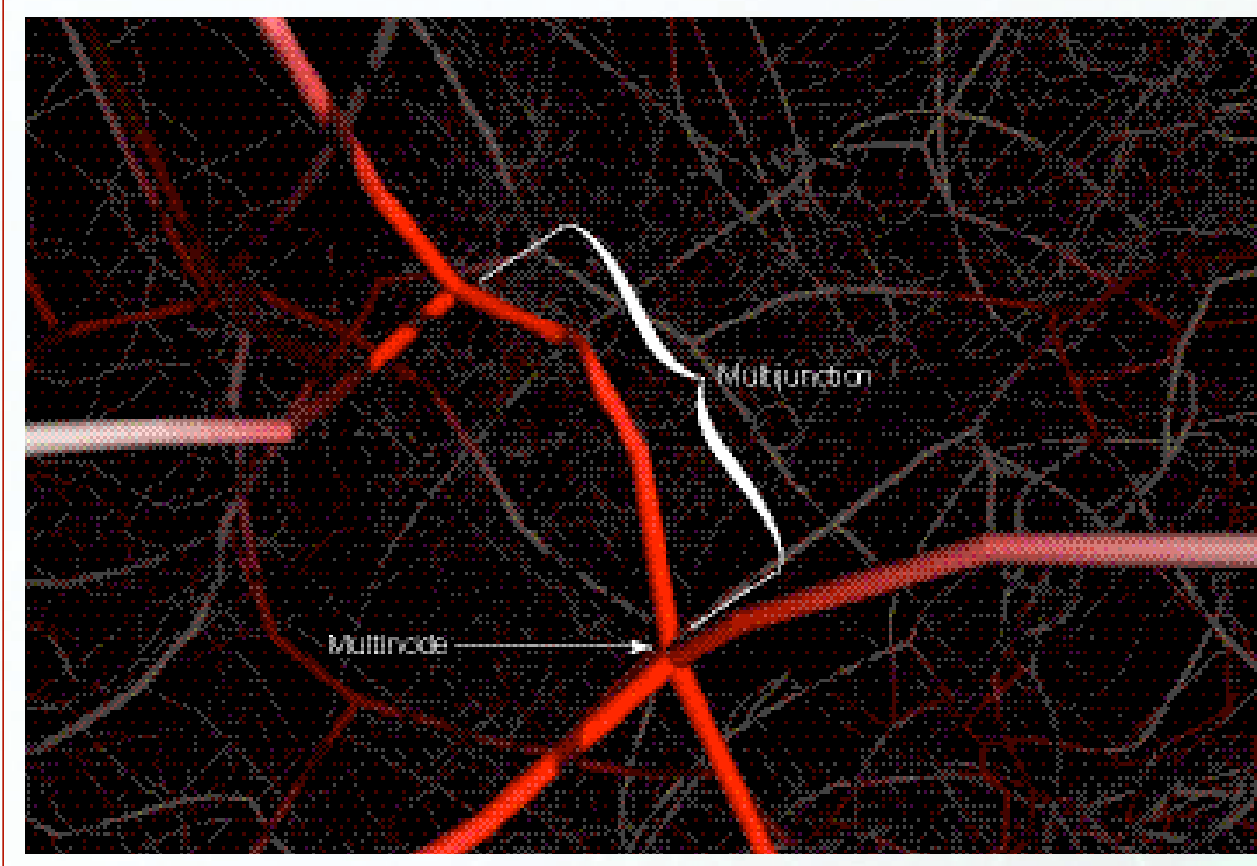

This new understanding is used to develop predictive models of materials performance under extreme deformation

Major Accomplishments in 2004 The classic dislocation theory is an analytical solutions for the energy and stress associated with dislocations. However, the solutions are singular an undefined on the dislocations themselves, making numerical calculations difficult. In previous attempts to develop a nonsingular theory, either the theory was too complicated to be useful for a numerical implementain or it was simple but we have developed ParaDis, In mesponse, parallel dislocation dynamics code that can follow large numbers of dislocation dynamics for a long enough time. ParaDiS incorporates a new, nonsingular continuum theory of dislocations that is both mathematically rigorous and analytically simple. In one of its early applications, the new theory provided an accurate description of the physics of

The new theory was instrumental in our recent discovery of multijunctions and multinodes that resulted from collisions of three or more dislocation lines.

Multijunctions are significant in that they tie together three and, possibly, more lines into very tight knots that serve as strong anchors for the whole network. A

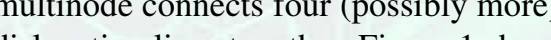
(inger 1 shows Figure 1. A dislocation dynamics simulation of
strain hardening in bcc molybdenum shows a fragment of the dislocation network. Binary junctions connect the mass of dislocation lines (grey). Multinodes (red) complicate the topology by forming a strong skeleton throughout the network.

For more information contact

VASILY BULATOV (925) 423-0124, bulatov1@ @llnl.gov

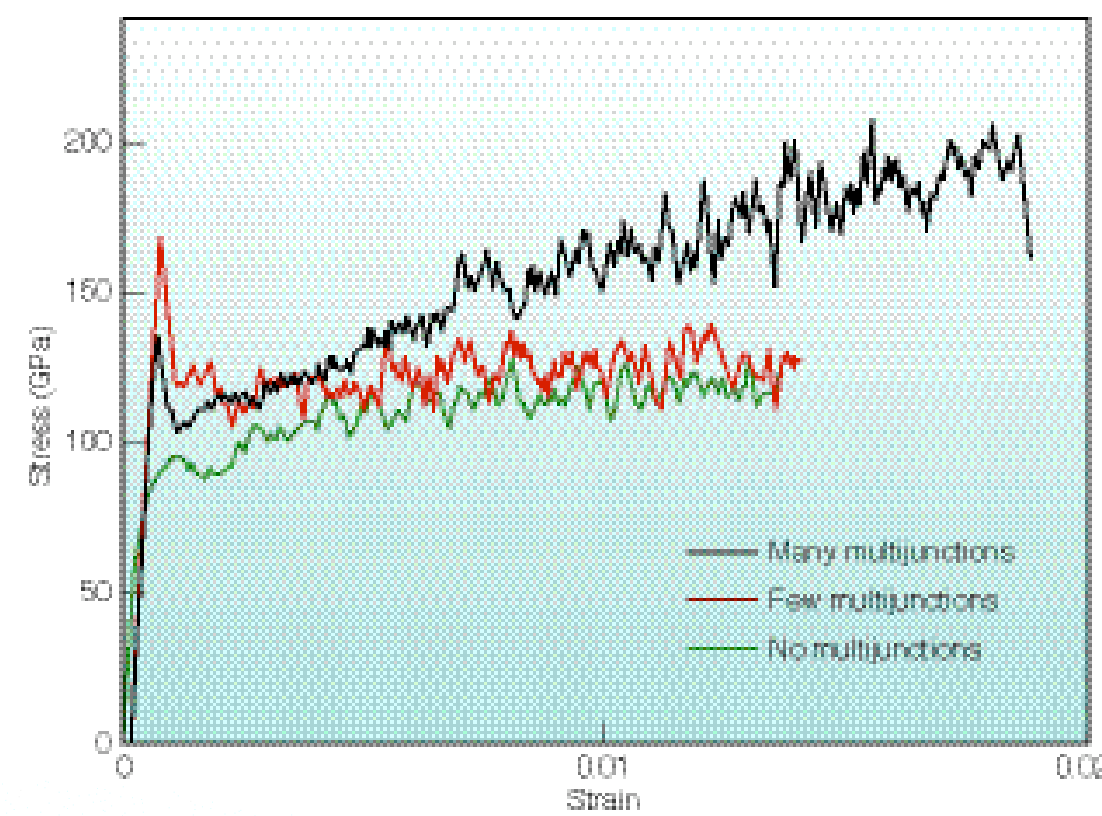

Figure 2. Stress-strain curves from a simulation on bcc molybdenum show that the formation of multijunctions significantly increases the hardening rate (line slope). Multijunctions that form in large numbers result in a well-defined slope (black) and are
contrasted with the nearly flat lines when only few (red) or no (green) multijunctions are formed. (Simulation image courtesy of M. Tang.)

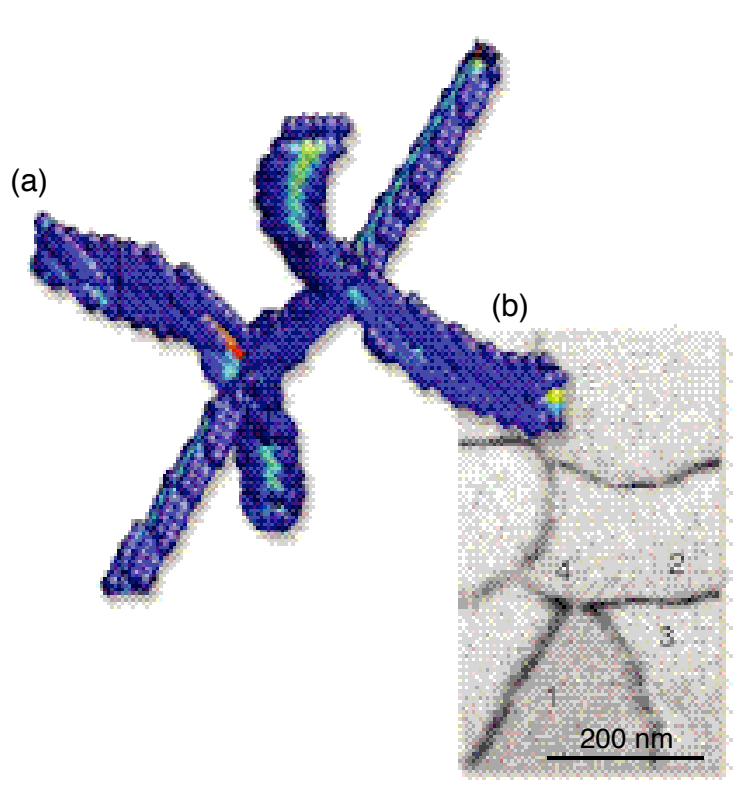

Figure 3. (a) An atomistic simulation based on an interatomic potential for molybdenum shows the formation of a multijunction. (b) A transmission electron microscopy
image shows a symmetric 4-node (numbered 1-4) and verifies the existence of multijunctions and multinodes.

a fragment of the dislocation network created in a very large dislocation
dynamics simulation. We found that ternary interaction in body-centered cubic (bcc) metals is three to four times strong than any binary interaction in the same metal and strong enough to pin
dislocation lines indefinitely.

\section{Scientific Impact}

Because the multijunctions in

dislocation networks are nearly

incesticted of metals. In particular, the existence of this element of dislocation microstructure offers an explanation to the unexplained large directional variation of strength observed in bcc single crystals.

In a series of large-scale dislocation dynamics simulations, we observed that the rate at which bcc metals harden duri plastic straining is defined, to a large extent, by the presence or absence of the of multijunctions was 2). The existence atomistic calculations and ultimately by experiments. Figure 3 shows an unmistakable signature of a multinode observed using TEM in a bcc single crystal of molybdenum deformed to 1 percent strain. The newly explained large variation in strenght results in the propensity or many bee alloys to localize their ultimate failure.

Related Publication V. Bulatov, et al,., "Scalable Line Dynamics in Paradis," conferenence.org/sc2004/schedule/pdis/spap206.pd

W. Cai, et al., "A Non-Singular Continuum Theory of Dislocations," J. Mech. Phys. Solicic,

\section{NEW FRONTIERS}

Having predicted and observed the crystals, we ned to understand the that many-body collisions play in other materials. We have already predicted the existence of multinodes in face-centered cubic crystals and believe that confirmation of their existence by TEM observations will follow.

Our preliminary results also suggest . phenomenology of dislocation icrostructure and strain hardent anticipate that new understanding of the topology of the highly entangled dislocation microstructures will compel researchers to generalize the models of material strength to include ternary and higher-order dislocation 


\section{Time-Resolved Observations of Electronic Structure}

The principal challenge of the emerging field of ultrafast materi dynamics hinges on our ability to characterize materials at both the time and length scales appropriate to the underlying physics. To probe the electronic an time and with femtosecond resolution, we are using $\mathrm{x}$-ray techniques, in combination with femtosecond lasers, to study the dynamics of metals during ultrafast laser irradiation.

These methods rely on the newest technological developments in $\mathrm{x}$-ray sources and detection systems.

Experimental protocols being develope to alution he ouly bing and spation

into contact with theoretical studies but extreme and dynamical conditions. also inspire new theoretical tools. We are By combining in-situ experimental interpreting our experimental results using diagnostics and new computationa multiscale modeling techniques that schemes, we are focusing on the account for electronic excitations and fundamental aspects of how materials hes seeted netherion fluid coupled to the ionic system.

Relevance to CMS Research Themes Advancing the fundamental scientific understanding of the dynamic behavior materials is essential for our nationalsecurity mission. Investigation of materials properties and performance is

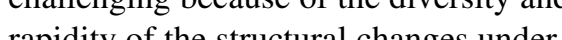

Figure 1. Photoelectron spectra for static versus laser-heated copper foil show how electrons respond increasing laser

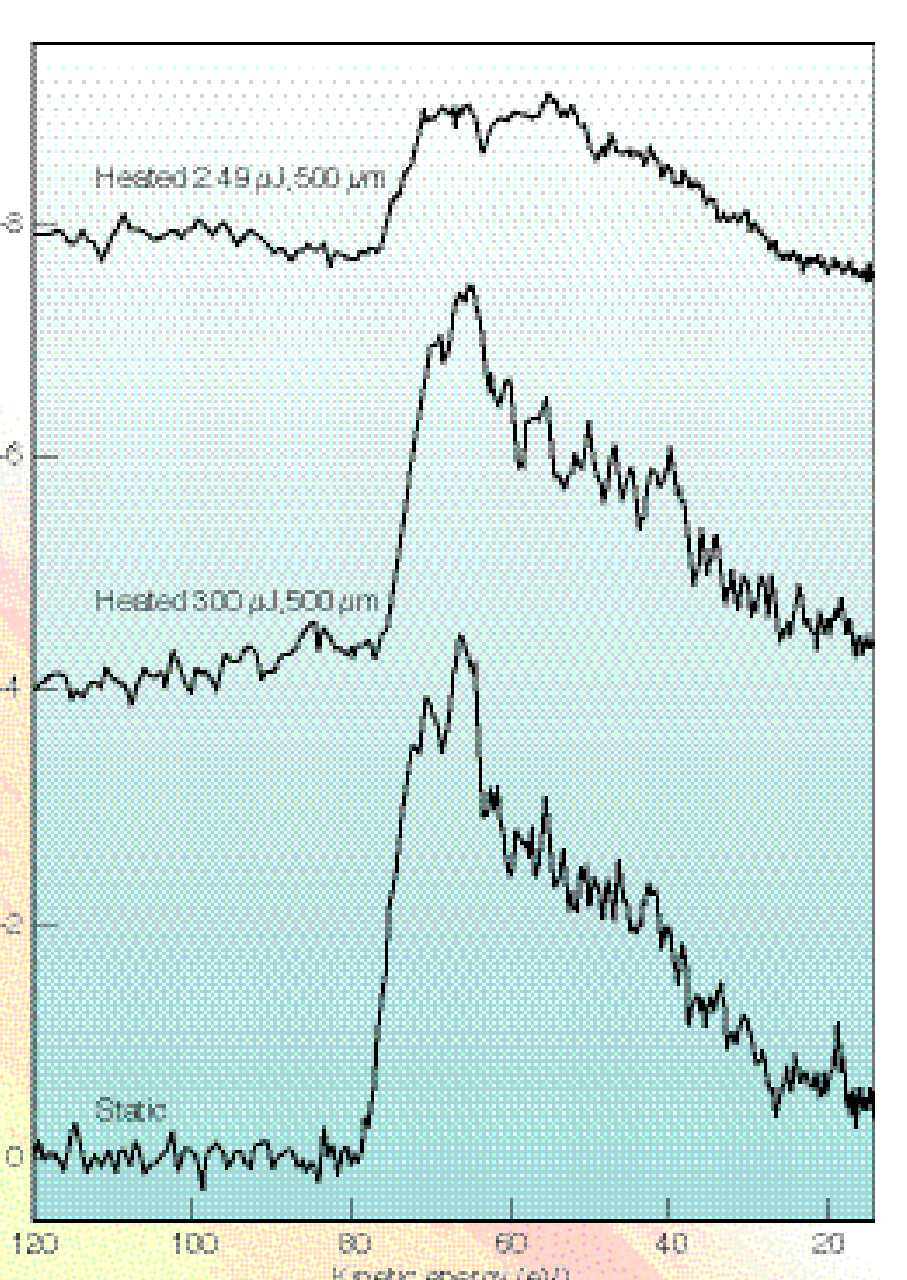

Major Accomplishments in 2004 To probe ultrafast changes in the materials, we use the compact multipu terawatt (COMET) x-ray laser to obtain the necessary high-photon flux (greater than $10^{12 / p u l s e), ~ m o n o c h r o m a t i c i t y, ~}$ picosecond pulse duration, and coherence. We have obtained the first photoemission foils showing changes in electronic

COMET is ideal for studying the process (electrons ejected from solids by radiation), as its short-pulse laser irradiation creates a non-equilibriu electron distribution at elevated temperatures, while the lattice remains a thin polycrystalline copper foils with $10^{8}-10^{9}$ x-ray-laser photons to measure how electrons respond to increasing lase intensity (Figure 1). We observe a strong $d$-state photoemission that corresponds to direct transitions from $d$-like occupied bands (high valency) to unoccupied bands (high conductivity) above the Fermi level (Figure 2). The data also indicate the hig density of filled $d$ states that are $2 \mathrm{eV}$ below the Fermi level.

Many other interesting and important features evolve with increased laser depopulation of the valence band $d$ states also creates vacancies in the conduction band, thus allowing interband absorption below the edge, in this case, from $3 d$ to $4 p$ this complex spectroscopy is fundamentally important in advancing our understanding of the dynamic electron time-of-flight photoemission

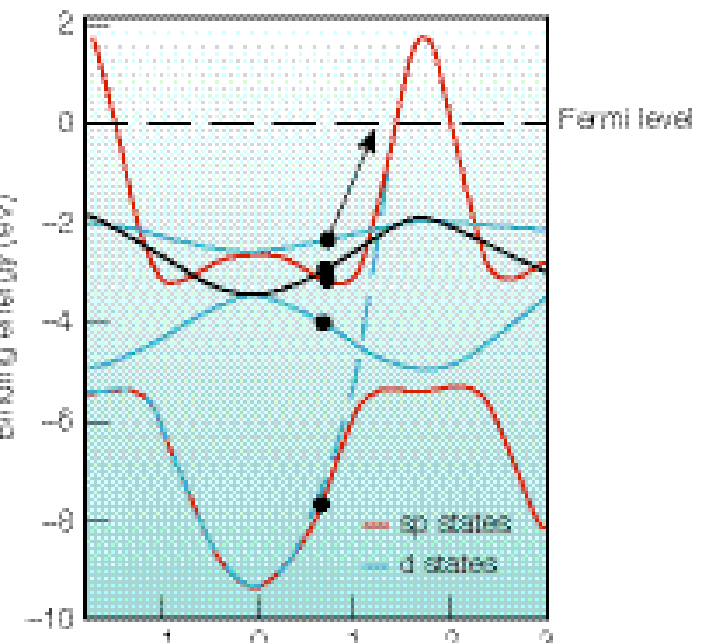

Marnention (s)

Figure 2. Schematic of photoemission process for copper ustrates a strong photoemission from direct transition

non-equilibrium processes at play that precede materials disassembly. We have also observed that the peak of
copper $3 d$ state shifts toward lower kinetic nergy (higher binding energy). Depopulation of the $d$ band is predicted We observed no broadenin of menner. Id state upon heating which implies a on-equilibrium distribution of occupied states, or smearing of the Fermi-Dirac electron energy distribution. Increasing the laser energy further by a factor of ten generates a strong electron signal before eaching the valence band maximum, Indicating the sample is in an ionized or In order to model laser interaction with dynamics simulations with a model for the dissipative dynamics of the electronion system to bring the two systems into equilibrium with each other (Figure 3). Specifically, we have formulated continuum description of the laser excitation and subsequent relaxation of the conduction band electrons and are applying firt-principles methodology for

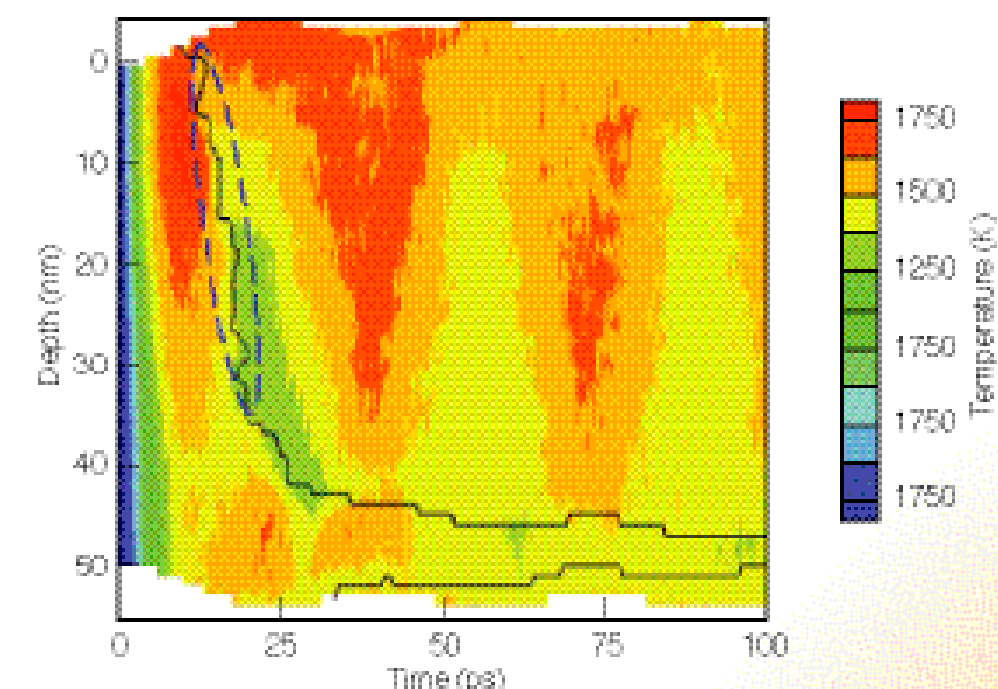

Figure 3. Temperature contour plot shows simulations of laser melting of a 50-nanometer nickel film. Strong electron-phonon coupling leads to a large
temperature gradient in the film. Ultrafast melting of a large fraction $(-35 \mathrm{~nm})$ of the film temperature g
is observed.

teristics. We are also extending the model to micrometer and larger length scout voids and the ablated surfaces. Scientific Impact These experiments demonstrate th Theresolved changes in electronic structure. This also included the first measurements of the disassembly dynamics of ultra-thin copper foil. The complementary relationship between these experimen and the newly developed, massively paralle hy photomechnis simulations of laser melting ad spallation of nickel and copper thin films. In collaboration with UC Berkeley, thi project provides a clear vision for ultraf examination of extreme states of matter.

Related Publications

A.J. Nelson, et al., "X ray Laser-Induced Photoelectron Spectroscopy for Single-State Measurements," App
Phys. Lett. 85(25), 6290 (2004).
L.V. Zhigilei, et al, "Computer Modeling of Laser
Melting and Spallation of Metal Targets," in Proc. Intl. Soc. Opt. Eng. 5448,505 (2003).

\section{NEW FRONTIERS}

When it becomes operationa in 2009, the Linac Coherent Light Source at the Stanford Linear Accelerator Center will be the world's first $x$-ray, free-electron laser. LLNL ef the LCLS will use the unique quality ter at high teate extreme slates of tor high-energy-density science, and to

CLS will be an important new interaction of intense $\mathrm{x}$ rays with matter. The unique attributes of the LCLS have the potential to revolutionize the experimental investigation of structural dynamics by directly following the time evolution of the electron density during the course of a biological, chemical, or to probe the ablation/damage process 


\section{Ultrafast Dynamics of Metal Deformation}

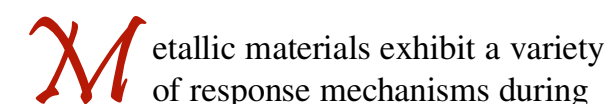
deformation processes, including dislocation production and motio diffusion, stress-induced phase transformations, and twinning in the crystal structure. Which mechanis depends upon temperature, pressure, strain rate. While the movement and interaction of dislocations may domin the overall response of metals, changes in the deformation mechanism create a fundamentally different environment which these dislocations move and interact. For example, the appearance of twinned lattice structure creates addition Chas terizing the mechan deformation under extreme conditions of pressure, temperature, and strain rate is challenging. Although detailed, real-tim observations at the relevant time and length scales of the dynamic process are not presently possible, we use detailed recovery-based observations on metals,

along with hydrodynamic and molecular dynamic simulations, to infer the conditions. Our goal is to investigate conte how differe to shocks and determine affect material properties. We will also compare material behavior across a wide range of loading conditions, utilizing multiple test platforms such as a gas high explosives, and lasers.

Relevance to CMS Research Themes Our stochpile stewardship nission demands a fundamental understanding of the behavior of solid metals under extreme conditions. To achieve this, new are required Correctly modeling metr response to shock-wave passage and subsequent high-strain-rate deformation requires an understanding of the materia response mechanisms across a wide ran of pressure, temperature, and strain rate. behavior must reflect the operative

deformation mechanism, while changes in this underlying mechanism may require changes in the form of the model. Detailed, recovery-based experimental observations play a vital role in these efforts. The experimental and modeling methodology we use will help advance models of metal deformation under extreme conditions.

\section{Major Accomplishments in 2004} We have conducted experiments utilizing different quasi-isentropic loading paths on a gas gun and a laser-based platform using both single-crystal and polycrystalline copper samples. Singlerecovered from the laser-bod have shown a gradual transition, from a dislocation-dominated to a twin-dominated (although still dislocation-assisted) deformation response, over a pressure range of $20-50 \mathrm{GPa}$, as illustrated in Figure 1. Polycrystalline samples have

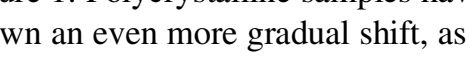
micrographs show residual deformation signatures from (b) $25 \mathrm{GPa}$, and (c) $42 \mathrm{GPa}$. $\mathrm{A}$ gradual shift from dislocation to twinning is evident.

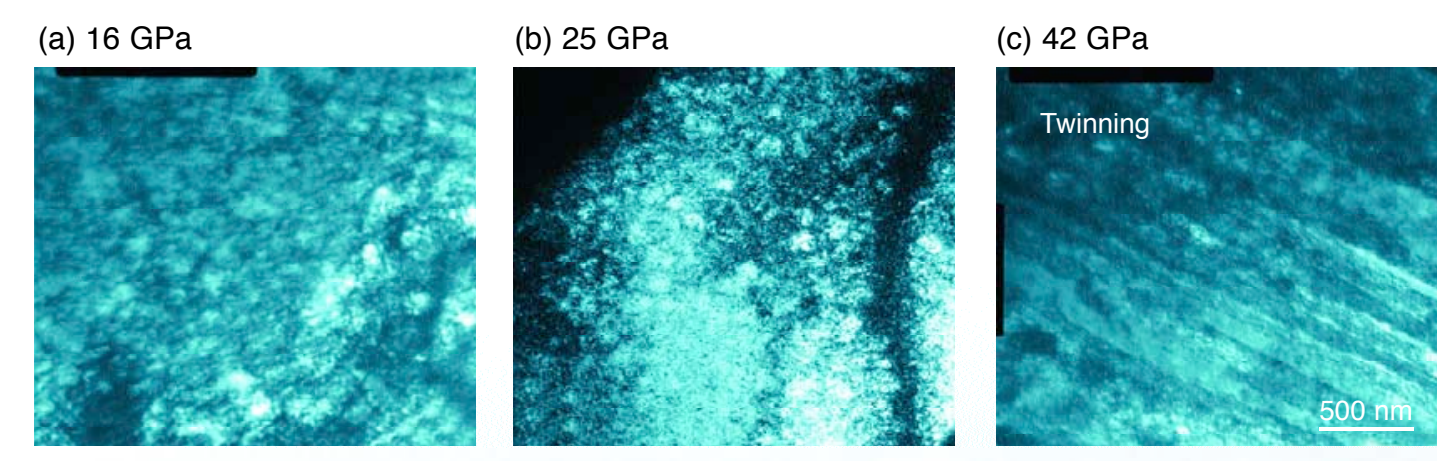
show (a) disisocation cells under shockless loading and

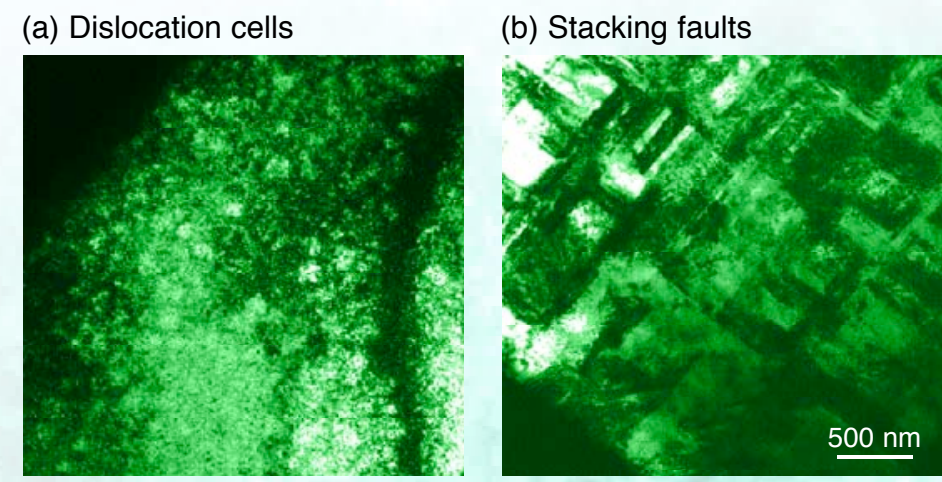

For more information contact

JAMES M. MCNANEY (925) 423-9335, mcnaney1@llnl.gov might be expected from the relatively few crystallites oriented favorably in an easily twinned direction. Analysis of the gas-gundriven material is currently in progress
Our laser-based system, the Ome Our laser-based system, he Onega laser at the Universty of Rochester, thermal transients. One advant has rap id platform is that the shock wave forms over a relatively small distance, which allows us to investigate both the isentropic (shockless) and shock response in a single sample at a fixed peak pressure. We have made the first observations of a remarkable change in material response over this transition. I isentropic loading, the residual dislocation cells, and in shock is dominated by stacking faults (Figure 2). Because the temperature pressure differences between these loading paths are very small, we conclu that this behavior is due to strain-rate

effects.

These experimental results support the olecular dynamics simulations conducted

as a component of this work. The results have indicated that there is a pressure theshold of $50 \mathrm{GPa}$ for twin formation the 20-GPa defect (Figure 3). These support the theory that the twin formation mechanism is one of coalescence where stacking faults on three adjacent planes join to form a "nanotwin" that may bsequently rapidly grow.

\section{Scientific Impact}

We have established a viable path Wward understanding the dynamic relevant materials. In a fechically found that for these specimen, wecovery experiments, a laser-based system minimizes the disturbance of the

microstructure and allows for retention of some deformation structures that would be unstable on more traditional platform Comparison of results across platforms provides a rational basis for assessing the

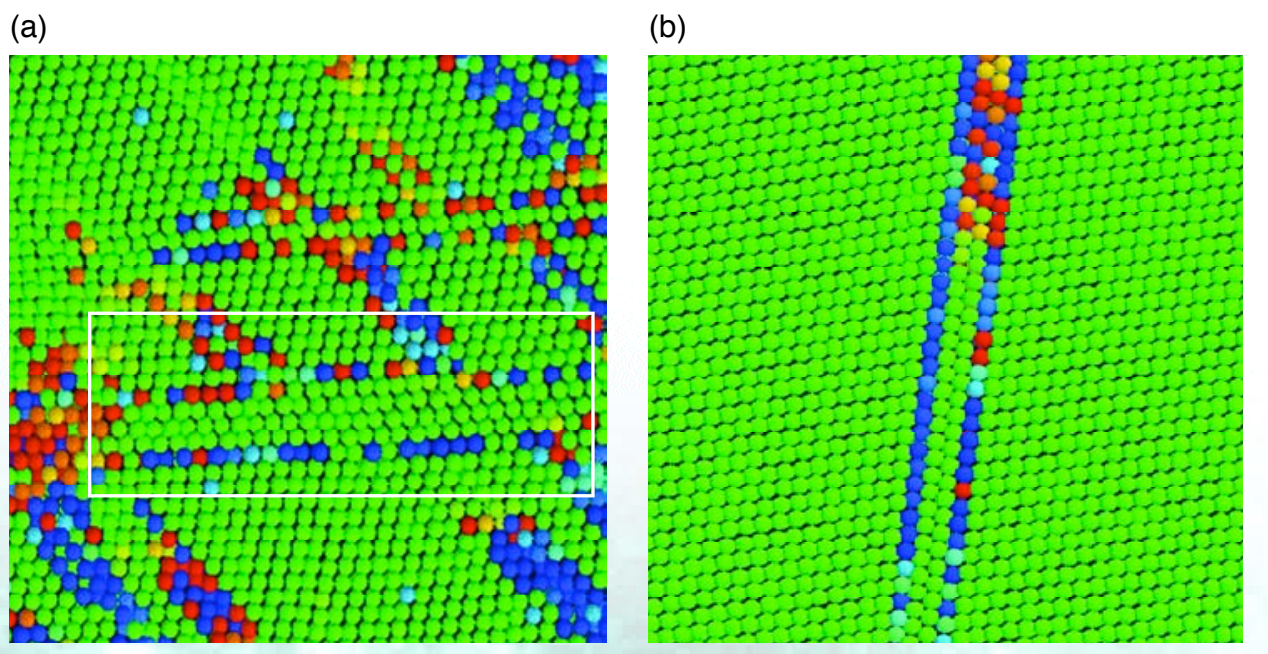

Figure 3. Molecular dynamics simulations show twin formation in single-crystal copper during (a) a 50-GPa shock in a perfect crystal and (b) a 20-GPa shock in a crystal containing a void hich collapses during shock passage). strategies to provide technically valuable strength data. Additionally, the use of multiple platforms, possessing distinct percesces in strain rate and hold time peak pressure, yields a more complese that take phace in the minis feare during these transients.

Related Publications L. Davila, et al., "Shock-Induced Vors," App. Phys. Lett, in press.

J.M. McNaney, et al., "High-Pressure, Laser-Driven Detomator or an Aluminum Alloy," Mettal. Trans. A. 35A, 2625-2631 (2004)

B.A. Remington, et al., "Materials Science under Extreme Conditions of Pressure and Strain Rat Mettal. Trans. A, 35A, 2587-2608 (2004).

NEW FRONTIERS

Looking ahead, we can hope to establish he upper and lower bounds on material constitutive response by

sample. In addition, the recovery

techniques used here may be leverag to provide containment capability in cases where it is desirable to contain the debris emitted from the test assembly.

Methodologies developed under this work are the first steps in providing valuable data to address the challenges (n) utrahigh pressures. For mexals at current technique for measuring metal strength results in the presence of a partially ionized plasma in contact with the sample surface. A heat shield is used to prevent the associated thermal wave from affecting the experiment. Multilaye deposits along with recovery-based experimentation will allow us to assess he effectiveness of these heat-shield materials. 


\section{Emergent Materials Properties of Plutonium}

he challenge of modern solid-state science is to identify new ways
matter is organized and to develop a matter is organized and to develop a
fundamental understanding of the physat fundamental understanding of the physica
principles involved in that organization. principles involved in that organization.
Solid-state science is currently confronted Solid-state science is currently
with what appears to be a new

whenomenology the quantum (QCP), which defines a phase transition a absolute zero temperature.

What is extraordinary about quantum criticality is that its influence is felt at temperatures far above absolute zero, possibly to the melting point in some materials. Indeed, many enigmatic properties of materials for which we only have empirical descriptions and little or no understanding $\mathrm{m}$
explained by QCPs

Plutonium, a material of special interest to LLNL, may be poised on the edge of just such a QCP as we study its physical changes caused by radioactive

decay. This possibility motivates us to investigate plutonium's thermodynamic properties at low or near zero temperature. Specifically, we are interested in lowtemperature magnetization and magnetic susceptibly as a fincion of self-cama

Relevance to CMS Research Themes Plutonium's technological importance stems from its nuclear properties, howev the physical properties of plutonium are defined by its electronic structure. While these properties are empirically well described, their fundamental origin is no. Characterization of the physical property changes acconnpanying radiation dan identification of a QCP will pro insight into plutonium's underlying $n$ and its organizing principles. This understanding is central to our work to the broader scientific challenge of

Figure 1. The superconducting quantum interference devic magnetometer uses a nor(cartridge brass). The inse shows a plutonium specimen. predicting material properties defined by electron correlations.

Major Accomplishments in 2004 Our principal goal is to learn how to "tune "plutonium in such a way as to properties change. We have devised a novel approach to introduce structural disorder through the accumulation of radiation damage (displaced atoms) in plutonium. Using a superconducting quantum interference device magnetometer and a unique sample holder (Figure 1), we have performed the first magnetic damage accumulation and annealing studies and crevious, mo results to those from based annealing studies.

The similarity to resistive annealing demonstrates the complex nature of defects on the magnetic properties of plutonium. This is the first observatio that the magnetic susceptibility of plutonium increases due to self-damage as plutonium decays, as illustrated in Figure 2 for two representative the magnetic susceptibility, the "excess magnetic susceptibility" or EMS. To understand more about this EMS we have developed a technique for measuring the time-evolving magnetization while carefully staying
below the temperature where radiation damage defects first become mobile, approximately $30 \mathrm{~K}$ for $\alpha-\mathrm{P}$ We have also Ksserved that the EMS eventually reaches a saturation point and
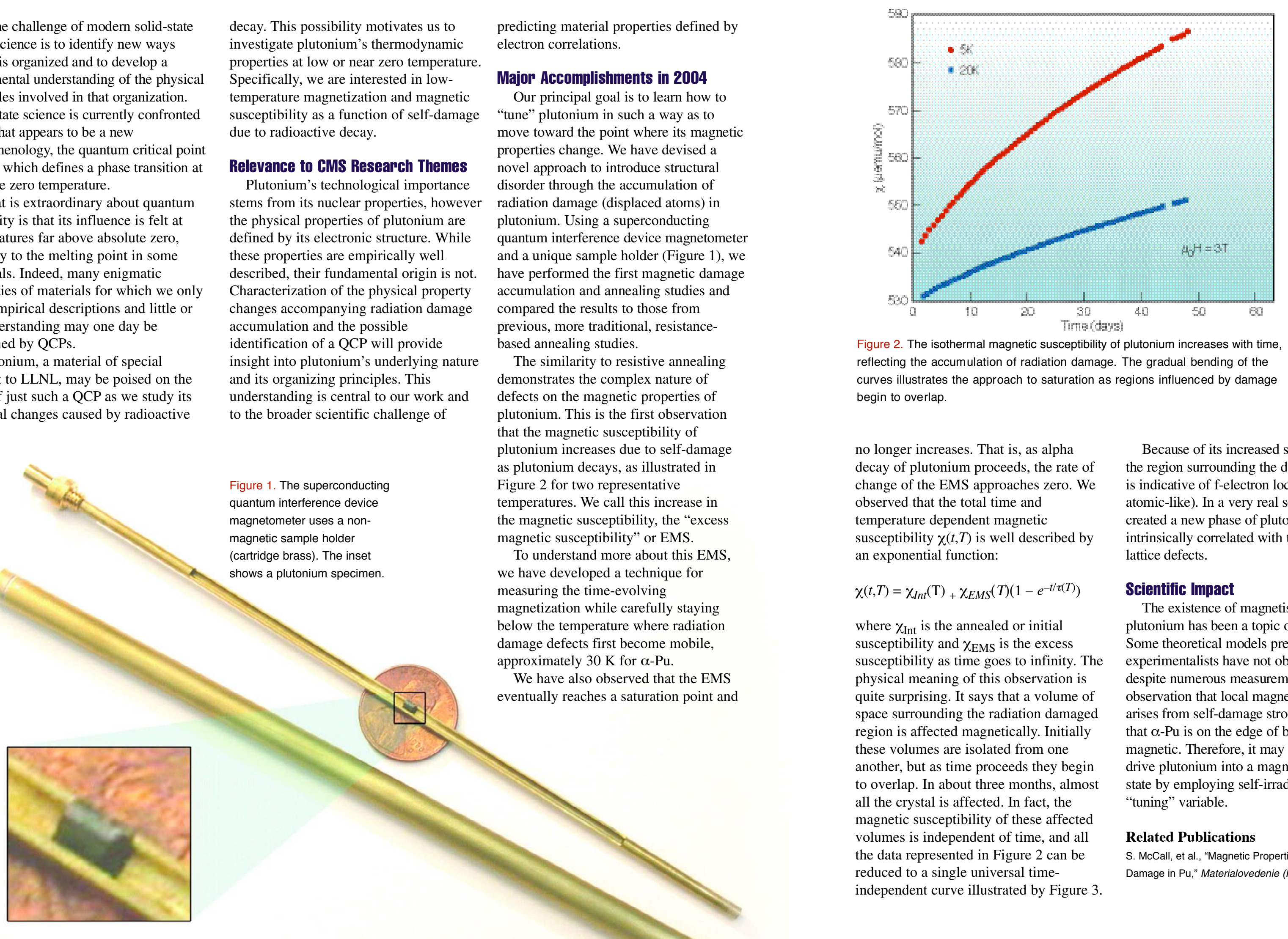

Figure 2. The isothermal magnetic susceptibility of plutonium increases with time, reflecting the accumulation of radiation damage. The gradual bending of the curves illustrates the approach to saturation as regions influenced by damage begin to overlap. no longer increases. That is, as alpha decay of plutonium proceeds, the rate of change of the EMS approaches zero. We observed that the total time and susceptibility $\chi(t, T)$ is well described by an exponential function:

$\chi(t, T)=\chi_{I n t}(\mathrm{~T})+\chi_{E M S}(T)\left(1-e^{-t / \tau(T)}\right)$

where $\chi_{\text {Int }}$ is the annealed or initial susceptibility and $\chi_{\mathrm{EMS}}$ is the excess susceptibility as time goes to infinity. The physical mening quite surprising. It says that a volume of rpace sus is these volumes are isolated from one another, but as time proceeds they begin to overlap. In about three months, almost all the crystal is affected. In fact, the magnetic susceptibility of these affected volumes is independent of time, and al the data represented in Figure 2 can reduced to a single universal time-
independent curve illustrated by Figure 3 .
Because of its increased susceptibility, is indicative of f-electron localization (mor atomic-like). In a very real sense we have intrinsically correlated with the pros is of

Scientific Impact

The existence of magnetism in purtonium has been a topic of controversy. despite numess have not observed it observation that local magnetic behavio aries from selfthat $\alpha$-Pu is on the edge of becoming magnetic. Therefore, it may be possible drive plutonium into a magnetic ground state by employing self-irradiation as a "tuning" variable.

Related Publication S. McCall, et all, "Magnetic Properties of Radiation Damage in Pu, 'Malerialovedennie (MTV), in press. Some theortical models predict it, but

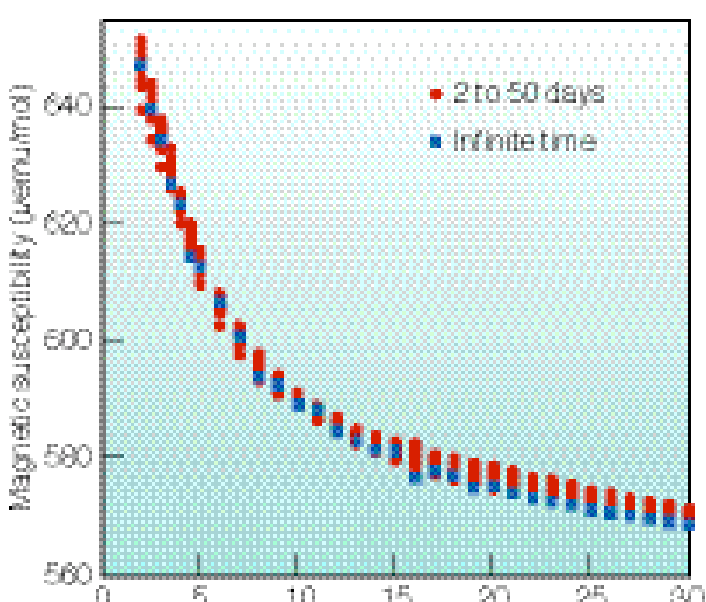

Temparchure (K)

Figure 3. We measure the effects of radiation damage on the physical properties of plutonium at different times universal time-independent curve. The elongated rod der points represent plutonium magnetic susceptibility over 2 days. The blue data points are the extrapolation to ite time.

Z.X. Zhou, et al,, "Transport and Thermodynamic Properties of $\mathrm{S}_{3} \mathrm{Ru}_{2} \mathrm{O}_{7}$ near the Quantum $\mathrm{Crntit}$ Point" Phys. Rev. B 69, 140409(R) (2004).

J. Custers, et al., "The Break-up of Heavy Electron Jan

\section{NEW FRONTIERS}

Acting like a negative pressure, the self-irradiation of $\alpha$-Pu disorders and locally modulates the atomic lattice. We are pursuing further measurements on both $\alpha$ - and $\delta$-Pu to help us map the he quantum critical point (OCP). In addition, by applying new techniques that allow a greater range of negative pressures, we expect to drive plutonium to a fully magnetic state and perhaps to the illusive QCP. Realization of a QCP may usher in a new understanding of the overall properties of plutonium and provide a new, albeit complex ground state. 


\section{Modeling the Many Faces of Water}

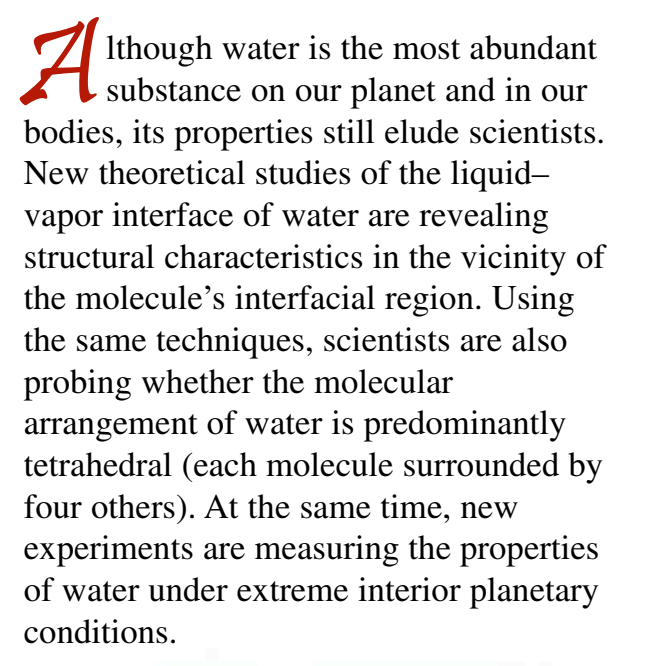

The challenge for theorists is to provide
a single model to describe water in all universal model that can reproduce and predict properties of water throughout its phase diagram. Three classes of models a covering both rigid and polarizable ions seem to perform best under ambient water model, one molecule at a time. A third model uses density functional theory (DFT) that contains explicit chemistry an charge transfer

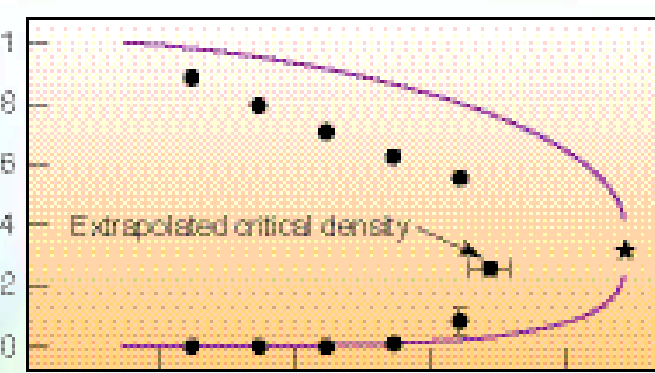

Figure 1. Phase diagram shows water at liquid-vapor interface (top curve), saturated vapor pressure (center), and heats of vaporization (bottom). Our ab initio model successfully describes, for the first time, the key thermodynamic variables of
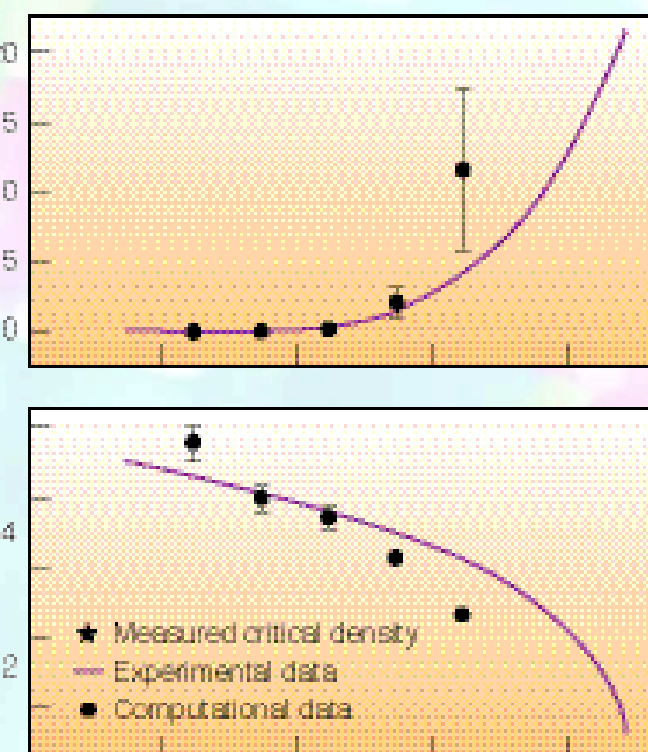

Temperctue
Relevance to CMS Research Themes Having a theoretical understanding of water has far-reaching implications to at least two CMS research themes. First, having the ability to model water in extreme environments is directly related conditions. Here, experimentalists and theorists are working together to examine molecular fluids that are exposed to high temperatures and pressures, and water is often used to help calibrate molecular systems. Second, water plays an

extremely important role in the emerging area of understanding biomolecular matter in relation to life sciences. In various environments ranging from the interior of proteins, having the theoretical tols understand water is positioning LINL become an emerging leader in the study of the complex and important chemistry that is present under ambient conditions. We have taken a multifaceted approach to modeling water. First, our

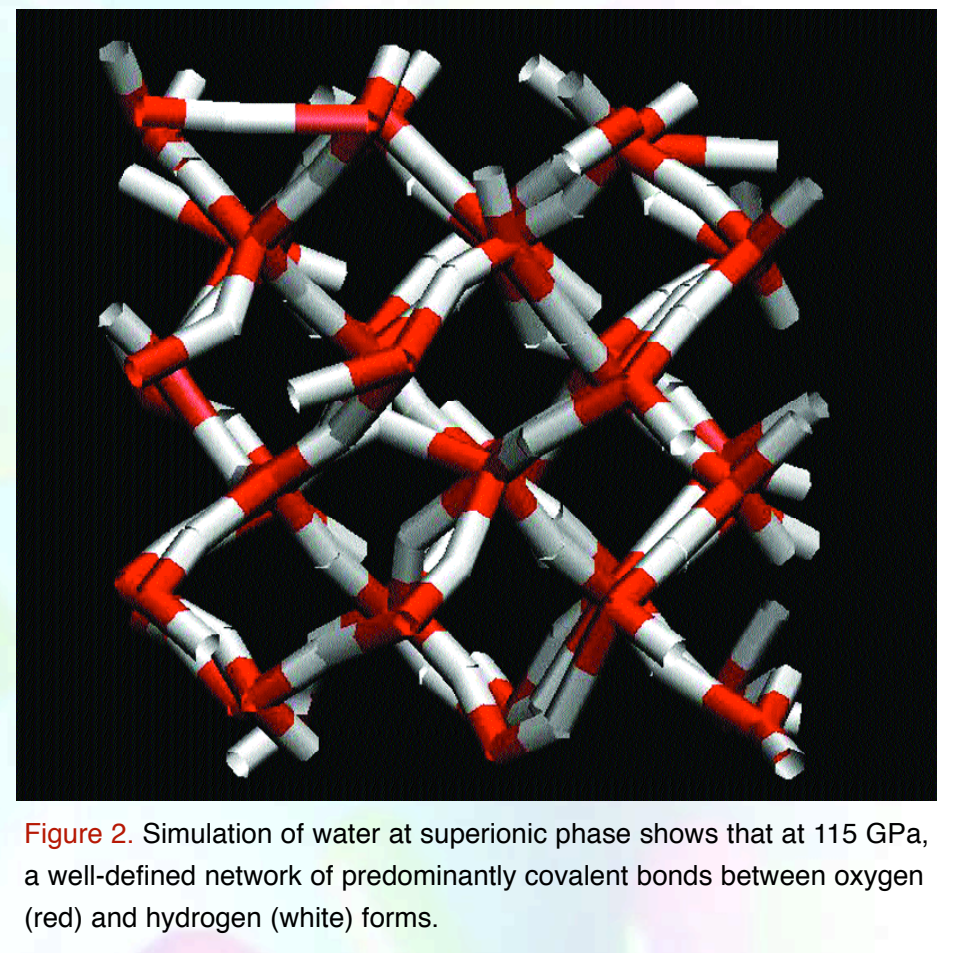

Major Accomplishments in 2004 model for the water dimer $\left(\mathrm{H}_{2} \mathrm{O}-\mathrm{H}_{2} \mathrm{O}\right)$ has proven to be highly accurate for larger clusters and the ambient liquid. This is the first time a model with gasphase spectroscopic accuracy has be phe to describe condensed-phase properties. This model has been used in simulations. We have also accomplished high-level quantum chemical calculation of the water pentamer, $\left(\mathrm{H}_{2} \mathrm{O}\right)_{5}$, with unique hydrogen bonding structures. To describe water outside of ambient conditions, we are performing pioneering calculations using both standard and new developed methodologies. Using DFT, we have performed the first-ever calculation containing 216 water molecules, and results agree with recent experimental These calculations have led to questions on the accuracy of DFT beyond $1 \mathrm{~g} / \mathrm{cc}$ and 298 K. Our group, in conjunction with many collaborators, has developed the fir Monte Carlo calculations using the DFT interaction potential, and we successfully computed the phase diagram of liquid water at the firs ace. These resils provide hint acturate assessment of (Figure 1).

Our simulations of water under the extreme conditions of planetary interior (2000 K and $115 \mathrm{GP}$ ) indicate that wate undergos several transformations. At glassy sase, white oxygen atoms form a glassy state, while the hydrogen atom between oxyen laptice by jumping extremely high hydrogen mobility, water is in a superionic state. Its presence had been predicted previously, but at significantly lower temperatures and pressures. Water in this superionic phase consists of extensive transient networks $\mathrm{O}-\mathrm{H}$ bonds, which are predominantly covalent (Figure 2). We find that at the superionic phase transition, molecular as molecules or ionic conductos a

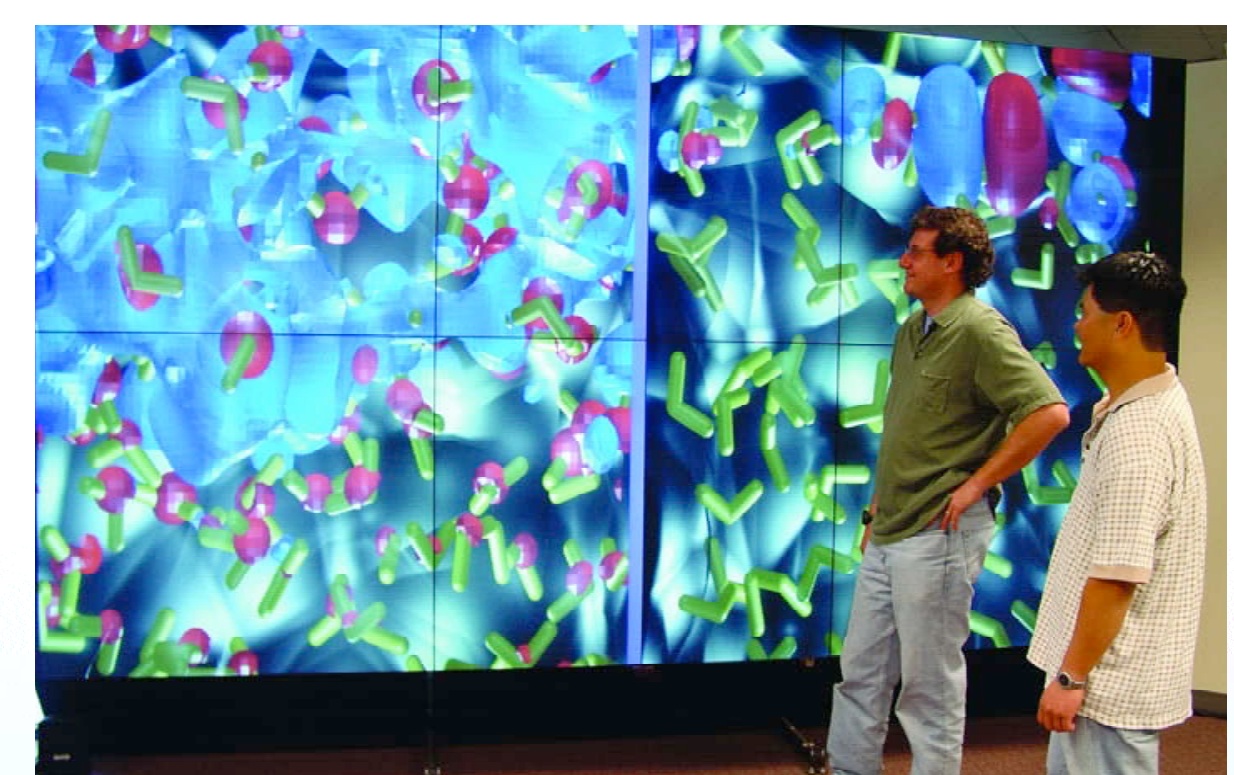

states.

Scientific Impact

Studying the structure of water under variety of conditions benefits many areas. Me density function theory hare beng the density function theory have been water has a stable liquid phase.

Furthermore, given that no single model of water will be able to describe water in all environments, a detailed knowledge the performance of more efficient mode will allow us to more confidently approach problems ranging from biology to atmospheric science.

Related Publications N. Goldman, et al., "A First-Principles Potentia Spectroscopy of Water Clusters" Phil A 363, 493-508 (2005)

I.F.W. Kuo and C.J. Mundy, "An Ab Initio Molecular Dynamics Study of the Aqueous Liquid-Vapo Interface," "Science 303, 658-660 (2004).

I.F.W. Kuo, et all. "Liquid Water from First-Principles: Investigation of Difterent Sampling Approaches,"
J. Phys. Chem. B 108, 12990-12998 (2004).

\section{NEW FRONTIERS}

New studies of water at the lquid-vapor interface have furthered our understanding that leads toward we will look an the chemich rutre, of water in environments that strongly modify its usual properties. In articular, we will examine acid-base conditions of high pressures and temperatures. We also plan to examine acid-base reactions in small water clusters, where surface effects play a dominant role in chemical reactivity. These studes will lead to more eficien hydrogn-bonded neturk stucture in water and gain fundamental understanding of its unusual chemical and physical properties. possible mode to describe water in all 


\section{Formation of Methane under the Earth's Mantle}

ethane is the most plentiful and a main component of th natural gas. However, oil and gas wells are typicially only drilled $5-10 \mathrm{~km}$ beneath the surface. At these depths, the interior pressures of the Earth are equ

We have collaborated with a team of Geophysical Laboratory, Harvard University, A rgonne National Laboratory, and Indiana University in South Bend to conduct a series of experiments and ve show

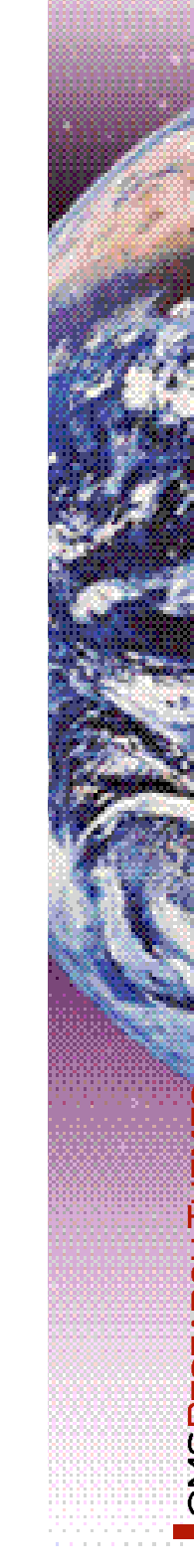

that methane forms under the high-pressur conditions that occur in the Earth's upp mantle at depths of $100-200 \mathrm{~km}$. The results of our studies indicate that mett processes under the Earth's surface.

\section{Relevance to CMS Research Themes} This research combines experiments and theory to understand chemical reactions under extreme conditions. Our be created from a non-biological source not just from the decomposition of living organisms. Also, our calculations show that methane is thermodynamically st

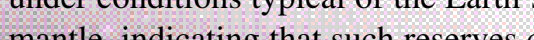
potentially exist below the Earth

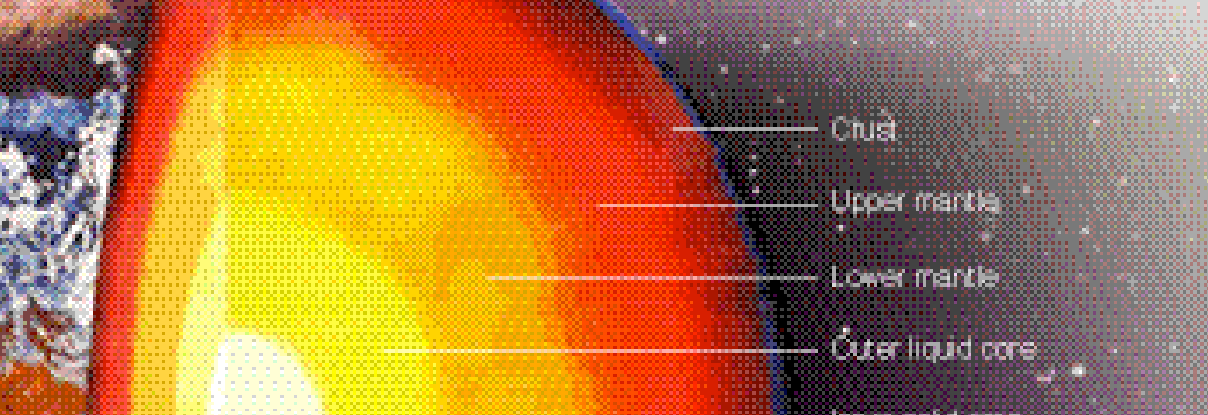

for millions of years. In addition to providing data that may indicate that the Earth holds limitless energy reserves, these studies will help to improve our ability to predict chemical processes explosive detonation.

Major Accomplishments in 2004 We conducted in situ high specifically designed to detect meth formation under geologically relevant conditions for the Earth's upper mantle. For the experiments, we created simultaneous high-pressure and cell by using both resistive heating (temperatures lower than $600^{\circ} \mathrm{C}$ ) and 1 los heating (temperatures higher than $1,000^{\circ} \mathrm{C}$ ) methods. Samples that contained natural $\mathrm{CaCO}_{3}$-calcite, $\mathrm{FeO}$-wüstite, and distille $\mathrm{H}_{2} \mathrm{O}$ were squeezed in the diamond anvil to produce the extreme conditions. Through a combination of Ram spectroscopy, synchrotron x-ray diffraction, and optical microscopy, we Perticularly, in situ Ran sin analyses. proved essential because of its high

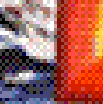

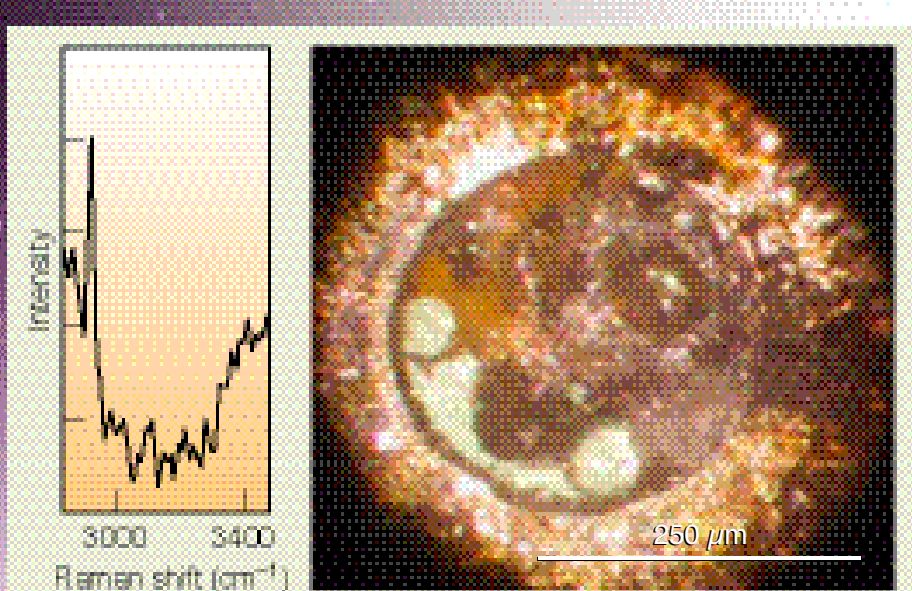

Figure 1. Methane-containing bubbles (right) are formed from Bubbles are visible near the bottom, left side, and slightly right of center O-H stretching vibrations because the bubble has displaced the surrounding $\mathrm{H}_{2} \mathrm{O}$. decompression to approximately $0.5 \mathrm{GPa}$ after laser heating at $5.7 \mathrm{GPa}$.

?

For more information contact

LARRY FRIED (925) 422-7796, fried1 @ llnl.gov

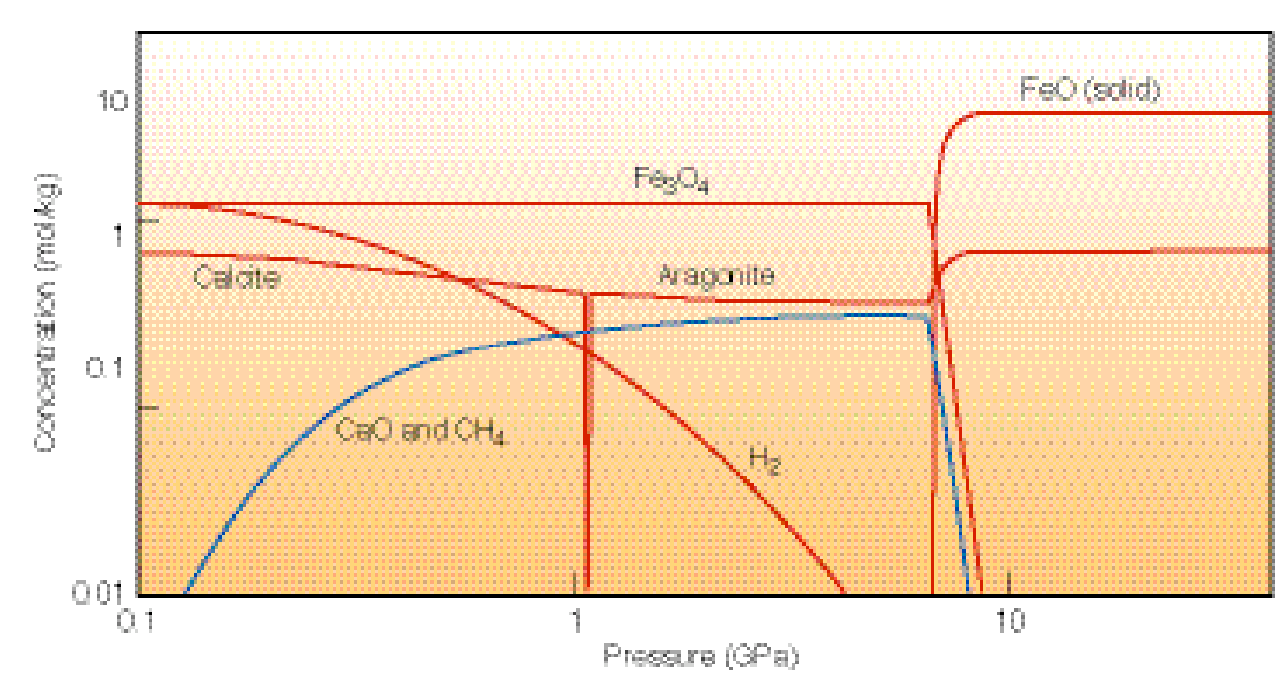

Figure 2. Our results
of thermochemical

of thermochemical

calculations show
that, at $500^{\circ} \mathrm{C}, \mathrm{CH}_{4}$

(methane) is

predicted to be

pressures up to

$10 \mathrm{GPa}$.

sensitivity to the $\mathrm{C}-\mathrm{H}$ stretching vibrations of molecular species; it was poorly crystalline phases. The synchrot x-ray diffraction provided a means to identify crystalline phases and helped determine the reactions.

As shown in Figure 1, hydrocarbonrich regions are found in our diamon anvil sample after laser heating at $\sim 1500^{\circ} \mathrm{C}$ at $5.7 \mathrm{GPa}$. The hydrocarbon is charp band at $2972 \mathrm{~cm}^{-1}$ athing by The sample exhibited considerable. heterogeneity. It is important to note that, in this experiment, the diamond anvils not show evidence of reaction with the sample material. Raman measurements laser-heated experiments typically are very challenging because the samples are
fine-grained and fluorescent after heating fine-grained and fluorescent after heating. and a layer of opaque material across the interior of the sample chamber from

optical measurements

We have obtained further insight into the chemical processes involved in highpressure methane production by conducting new thermochemical modeling. Our aim is to address the temperature and pressure dependence of the high-pressure formation of methane is if this is an experimental artifact (laser versus resistive heating). By using our Cheetah thermochemical code, we calculated the high-pressure chemical equilibrium. Figure 2 shows that meth is thermodynamically stable at $500^{\circ} \mathrm{C}$ at pressures up to $10 \mathrm{GPa}$.

Scientific Impact

The Earth's mantle is a dense, hot $2,900 \mathrm{~km}$ thick. It contains more iron magnesium, and calcium than Earth's crust, and it is hotter and denser because temperatures and pressures inside the Earth increase with depth.
Because of the firestorm-like temperatures and crushing pressures in differenty than they do on E behave very At temperas the on Earth's surface. have found optical changes indicative of methane formation, and at temperatures above $1500^{\circ} \mathrm{C}$, the carbon in calcite form carbon dioxide rather than methane. This implies that methane may be present at depths between $100-200 \mathrm{~km}$, and it may be more prevalent in the mantle than previously thought. Because of the vas size of Earth's mantle, hydrocarbon reserves in the mantle could be much
Related Publications

H.P. Scott, et all, "Generation of Methane in the Earth's Mantle: In Situ High Pressure-Temperature Acad. Sci. 101, 39 (2004).

N. Wade, "Petroleum from Decay? Maybe Not, Study Says," The New York Times, September 14, 2004.

\section{NEW FRONTIERS}

Our research combining experiments with theoretical calculations indicates hermodynamically stable und conditions typical of the Earth's mantle. We intend to study the phase diagram and stability of other simple molecular compounds under high-pressure and emperature conditions. We are extending techniques to study water under conditions of giant planetary interiors. For example, we use first-principles molecular dynamics calculations and Raman spectroscopy to investigate the existence of a novel "superionic" phase of water. In this phase, oxygen atoms are fixed whit hydrogen atoms diffuse freely. These lechniques can improve our ability to predict a host of chemical processes previously unattainable. 


\section{Probing Materials under Extreme Conditions}

$W_{\text {processes that occur in our }}^{\text {ost of the chemical and physical }}$ universe take place under conditions tha seem extreme to us on Earth. Nevertheless, the study of these processes under controlled conditions in the laboratory will yield great insight as well of physical properties that are of direct importance to many diverse disciplines. There are many systems whose behavior under extreme conditions is of great relevance to both fundamental science and also to the essential mission of LLNL. The present challenge is to develop the corresponding experimen gate and model these

Relevance to CMS Research Themes The development of effective diagnostic tools to elucidate the nature of extreme-conditions phenomena has been at the core of Livermore's mission. The overarching aim of our research is extend the range of these tools to conditions that have hitherto pros
investigation. The results of

our work can be applied to unique paramount concern to national security.

Major Accomplishments in 2004 In spite of recent advances in diamond pressures exceeding $100 \mathrm{GPa}$ atic challenge. We have successfully demonstrated the utility of a new instrument that employs the technique impulsive stimulated light scattering (ISLS) to measure acoustic velocities to pressure in excess of one million almospheres. Developed by Liverme scientists, his transparent and opeque matials in or to determine their elastic properties.

ISLS and Raman Spectroscopy: Our system enables us to make acoustic wave velocity measurements under previously provides exceptionally precise and

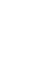
anvil cell techniques, accura pibration inaccessible conditions. The instrume accurate data. We have demonstrated its ability to determine either the entire

elastic tensor or selected tensor elements of single crystals (including germanium and cobalt) and also the shear and compressional elastic moduli of
polycrystalline aggregates (including tantalum and iron).

Using ISLS and our unique Raman spectroscopic system, we have determine the sound velocities of hexagonal closepacked iron to pressures approaching $120 \mathrm{GPa}$ - corresponding to depth near the mantle-core boundary in the Earth's interior. Once thermodynamically scaled clearly imply that the Earth's solid inner also agree with vare ing. These resuls through shock-wave measurements. Our study of cobalt-analogous in some ways to iron-is also important for a better understanding of the compositio and structure of the Earth's interior. For the first time, we have obtained direct measurements hat indicate a pressureinduced collapse of magnetic ordering in cobalt that drives it to a nonmagnetic sta physical properties. This behavior is consistent with a prior theoretical mode postulating magnetic suppression.

Ring Compression: We have developed a combined experimental and computation technique to determine the coefficient of high pressure in the diamond anvil cell. A an example, we have performed the firstever application of the ring-compression
test to determine the coefficient of friction between molybdenum and diamond under high hydrostatic pressure (Figure 1). This type of knowledge is useful for the determination of yield strengths of materials under pressure and for fully interpreting the results of various types of experimental investigations of highpressure rheology to the Earth's core temperatures, our dat friction between diamond and metal under with theoreticians as well as computatio scientists, we continue to apply novel diagnostic techniques in the discovery of many never-seen-before materials properties that can be validated by both For example, using Raman scattering and laser heating, we have observed for the first time the existence of a novel superionic phase of water above pressures of $50 \mathrm{GPa}$ and temperatures of approximately $1000 \mathrm{~K}$ (Figure 2). Superionic water could be plentifu inside giant planets such as Neptune. Water under these conditions should be highly conductive, hanks to mobile lattice of oxygen ions.

We have also recently used our
then of combined Raman/laser heating system to extend the melting curve of nitrogen to much high pressures than previously known and to investigate the behavior of cubic boron nitride at pressures of to $40 \mathrm{GPa}$ and temperatures in excess

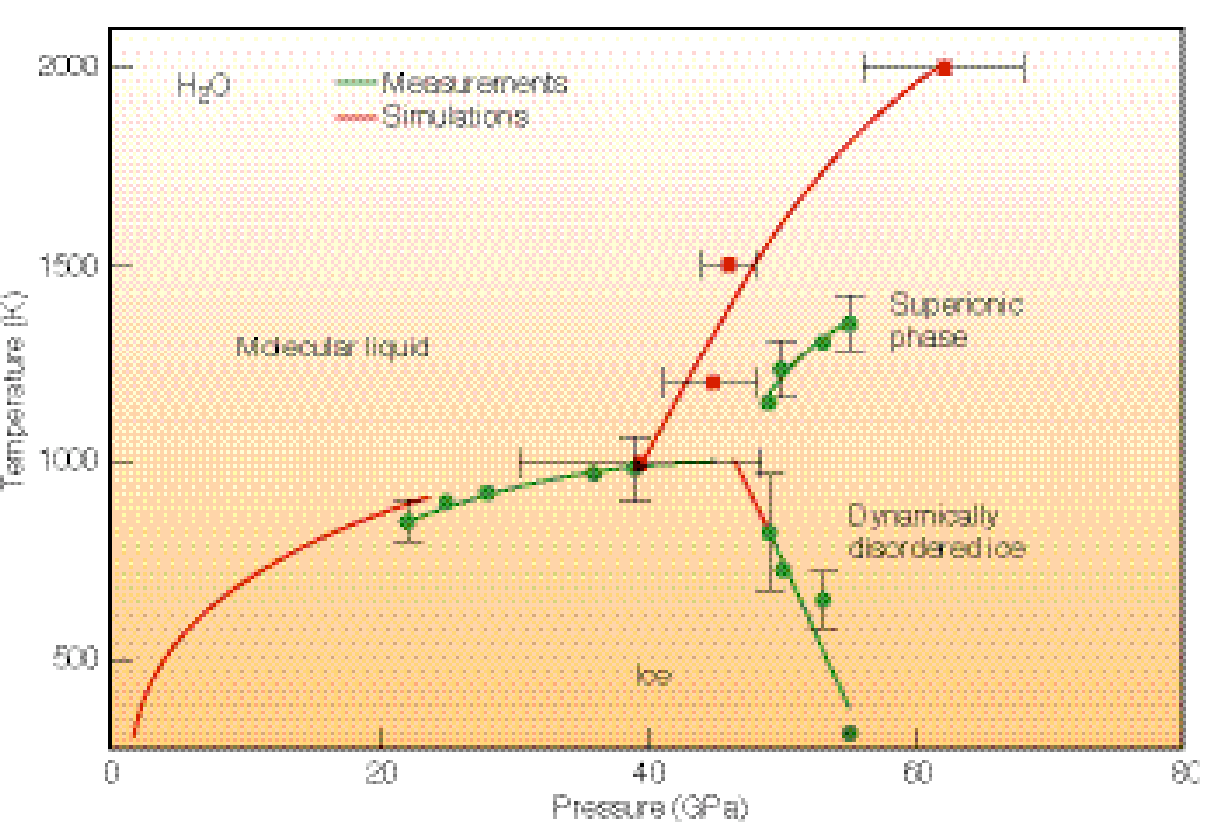

Figure 2. Phase diagram of $\mathrm{H}_{2} \mathrm{O}$ shows that under high temperatures and pressures, water exists in a superionic phase. For the first time, experimental measurements (green) and computational data (red)
have verified its existence.

of $1700 \mathrm{~K}$. We have used the results of first latter investigation to develop the Using loserate scale above $1000 \mathrm{~K}$. synthesized the world's second known noble metal nitride: iridium nitride. Th compound forms above $47 \mathrm{GPa}$ and of the diamond cell to synthesize novel materials.

Scientific Impact

Advancing our understanding of mechanical properties at extreme conditions allows us to develop the scientific challenges. For example, the hear moduli of many actinides above few tens of GPa or at simultaneous There is a lack of experimental data o how the magnetic properties of these metals coupled with strength paramete at high pressure-temperature conditions neither has it been accurately determin how yield strength responds to
Laser Heating: Working side-by-side

pessure shows the effective plasit $90 \mu \mathrm{m}$ equalizes pressure around the ring. 


\section{Structure Control in Sol-Gel Materials}

$\int$ ol-gel is a chemical synthesis process $\int$ that offers a versatile approach to the design of advanced materials for a range of applications, including catalysis, separatio technology, ceramics, sensors/detectors, a nanoelectronics. In the sol-gel process, reaction precsizens transfom into with one another to form a threedimensional solid network (gel) This technique provides compositional and structural control on the nanometer scale as well as access to various forms of the desired material, such as powders, film fibers, and monoliths. We have developed a new sol-gel method that greatly expands the types of exploit this new technique, however, we need a fundamental understanding of the mechanisms that drive the chemistry and how these mechanisms influence the physical properties of sol-gel material. To obtain these insights, we are utilizing nuclear magnetic resonance (NMR) spectroscopy as an in-process characterization tool to monitor particle formation and organization in these nove task because the technique can track transformation in either liquids or solid in an elementally selective and no destructive manner. In addition, NMR observables are directly related to kinetic and thermodynamic processes that control macromolecular formation, which, in turn, affects the bulk physical properties.

Relevance to CMS Research Themes Ongoing development of advanced science and technology efforts at Lawrence Livermore. CMS supports this endeavor through the investigation of chemistry and materials under extrem conditions. The synthetic methods we have developed provide the ability to

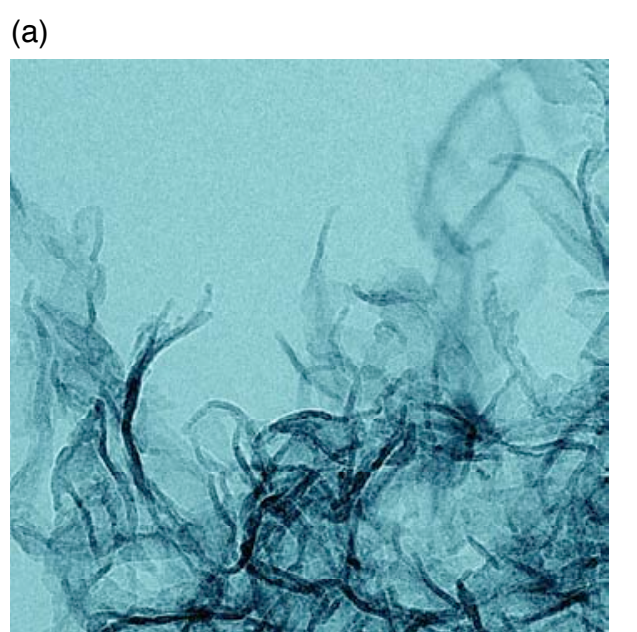

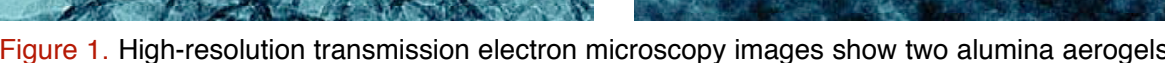
prepared using different ransmission electron microscopy images show two alumina aerogels aerogels have identical chemical composition but very different materials properties.

examine new or alternative synthetic routes and meet design criteria such as composition, purity, yield, and physical properties. Applying sol-gel chemistry to materials is part of a broad effort that includes preparing and characterizing wide range of designer materials, such as foam, stronger and tougher nanoceramics, and energetic nanocomposites.

Furthermore, this work contributes to the fundamental science and technology of sol-gel chemistry.

Major Accomplishments in 2004 By combining sol-gel chemistry an advanced NMR techniques, we gaining detailed insight into the relationship between synthesis and materials. We have used NMR to structure formation in two types of aluminum oxide $\left(\mathrm{Al}_{2} \mathrm{O}_{3}\right)$ aerogels. $\mathrm{B}$ changing a single synthetic variablespecifically, the anion of the sol-ge precursor-in the sol-gel reaction,

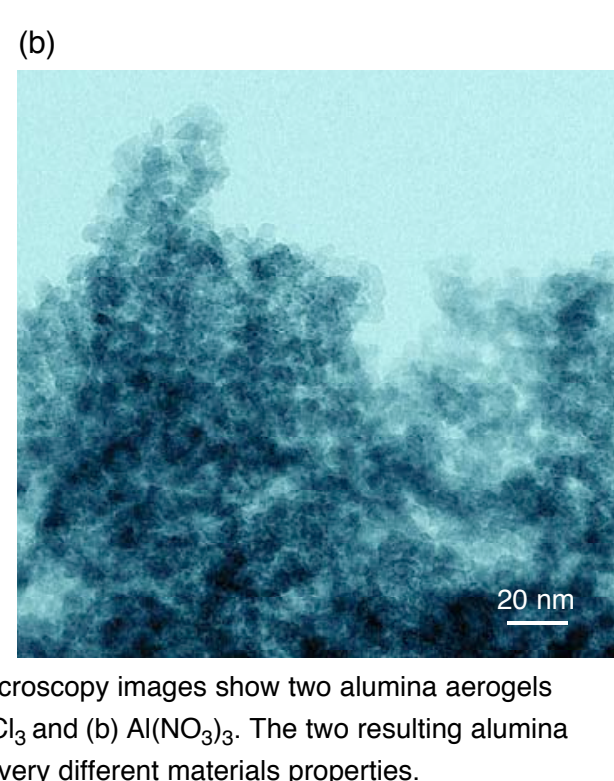

aerogels that have identical chemical composition can be generated to possess mechanical properties. The materials shown in Figure la display fibrous morphology that is substantially stronger than the more random cluster aggregatio To in Figure $1 \mathrm{~b}$.

actions that occur solid-ste NMR techniques to follow te kinetics of the nucleation and growth of the sol-gel architecture for both types of the aluminum oxide. NMR experiments for ${ }^{27} \mathrm{Al}$ provide information regarding aluminum speciation (4-, 5-, or 6coordinate $\mathrm{Al}^{3+}$ species) in the sol-gel building blocks, as illustrated in Figure 2 which building blocks.

We are also characterizing the transformations that occur in the gel state (mixture of both solid and liquid phases) of the sol-gel reaction process (Figure 3 ). These gel-state NMR experiments represent the first time this technique has

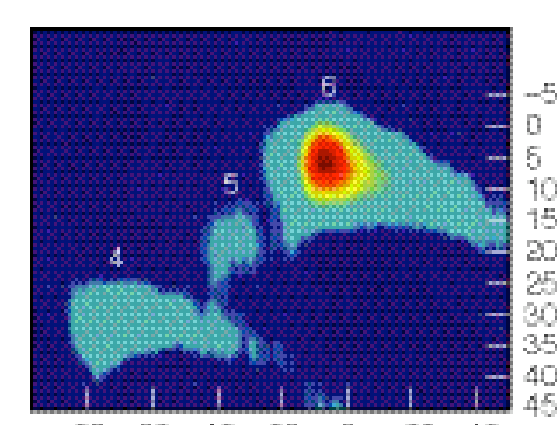

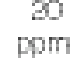

igure 2. Two-dimensional2zAl nuclear magne fic resonance spectra provide intormation regarding three species (4-, 5-.

been applied to sol-gel transformations in non-silica systems and provide a crucia link between traditional solution and solid-state experiments. We will estab the relationships between the initial reaction conditions and the resulting butk properties of the sol-gel materials. Ultimately, his knowledge will be applicable to the fabication of other expand the technological potential of gel science.

Scientific Impact broad range of fields, from electronics a biology to energy and physics. Advances in these areas reque new mods for the preparation of nanomaterials with desi Therefore, synthesis and processing of nanomaterials are an essential aspect of nanotechnology. Sol-gel chemistry produces materials that are inherently nanostructured and thus holds the potential for technological innovations in a number of important areas. A fundamental understanding of the mechanisms associated with sol-gel chemistry will lead to the design of new nanostructured materials as well as further our understanding of structure-property

Related Publication T.F. Baumann, et al., "Synthesis of High-Surface-Are Precursors," Chem. Mater. 17(2), 395-401 (2005)

I.L. Moudrakovski, et al., "Probing the Geometry and Interconnectivity of Pores in Organic Aerogels Using "Hypopolanzed raxe NMR Spectroscopy," J. Am Chem. Soc. 126, 5052-5053 (2004).

$$
\text { al }
$$

\section{NEW FRONTIERS}

Advancements in sol-gel chemistry are leading to new applications that are continuing to evolve. For example, we mew materials used in high-energy-density chysicas experiments, an area that is We are using our sol-gel process to prepare materials for sensors/detecto catalysis, and energetic materials. We also have burgeoning efforts in the areas of ceramics, hydrogen storage, and waste remediation, all of which involve novel nanostructured materials prepared by using sol-gel chemistry. Our untrale goal in this efion is to establish cond in this eftor is to analogous to those that have been used to develop structure-property relationships in organic polymer systems. 


\section{Tracing lodine-129 from Nuclear Fuel Reprocessing Facilities} odine exists in many organic and
inorganic chemical forms that are thermodynamically stable in multiple oxidation states. Its complex chemistry renders it ideal to use as a tracer. Isotopic tracers are an important component for because they can be used as fingeprint to help identify and characterize nuclear fuel reprocessing activities. Iodine-129, in particular, is produced as a result of nuclear reprocessing. Characterizing ${ }^{129} \mathrm{I}$ speciation in the environment is the key to understanding its mobility and absorption into the environment. The current challenge to interpreting ${ }^{129}$ levels is the lack of knowledge about its residence We are developing new analytical echniques to improve measurements of key parameters used to predict and mode dispersal of ${ }^{129}$ I from reprocessing emissions in relevant environmental matrices. The results of this research will provide the scientific basis for predicting
the transport and biological uptake of 129 released from fuel reprocessing facilities and will allow unambiguosi

Relevance to CMS Research Themes basis for extending the geographic range for effective sampling, choosing the bes sample matrix type and determining the timing of ${ }^{129}$ I releases. Together, these will form a powerful tool for monitoring the source and history of 12 emissions fron nuclear fuel reprocessing facilites radionuclide in the long-term dose assessment for the Yucca Mountai nuclear-waste disposal site and the Nevada Test Site. In addition, our studies advance areas of nuclear science for human health. For example, at the he of dose estimate uncertainties are unknowns regarding the chemical form and biologic pathway of ${ }^{12}$ I to the
Major Accomplishments in 2004 We use x-ray absorption fine-structure (XAFS) technique to identify speciation of iodine in solids and to characterize the coordhats information. This is the firs environmas iodine near-neighbor information. In addition to measuring the oxidation state and local bonding geometry of iodine, XAFS quantifies the number and species of iodine atomic neighbors as well
as the iodine-neighbor bond lengths. We have measured iodine XAFS spectra fo number of environmental samples, including iodine-containing marine minerals. XAFS spectra can be sather into several groups, including inorganic and organic chains, organic ring molecules, and mineral structures. The results shed light on the nature of the carbon-iodine bond, which determines residence time for organo-iodine compounds during biogeochemical
cycling of biological, geological, and

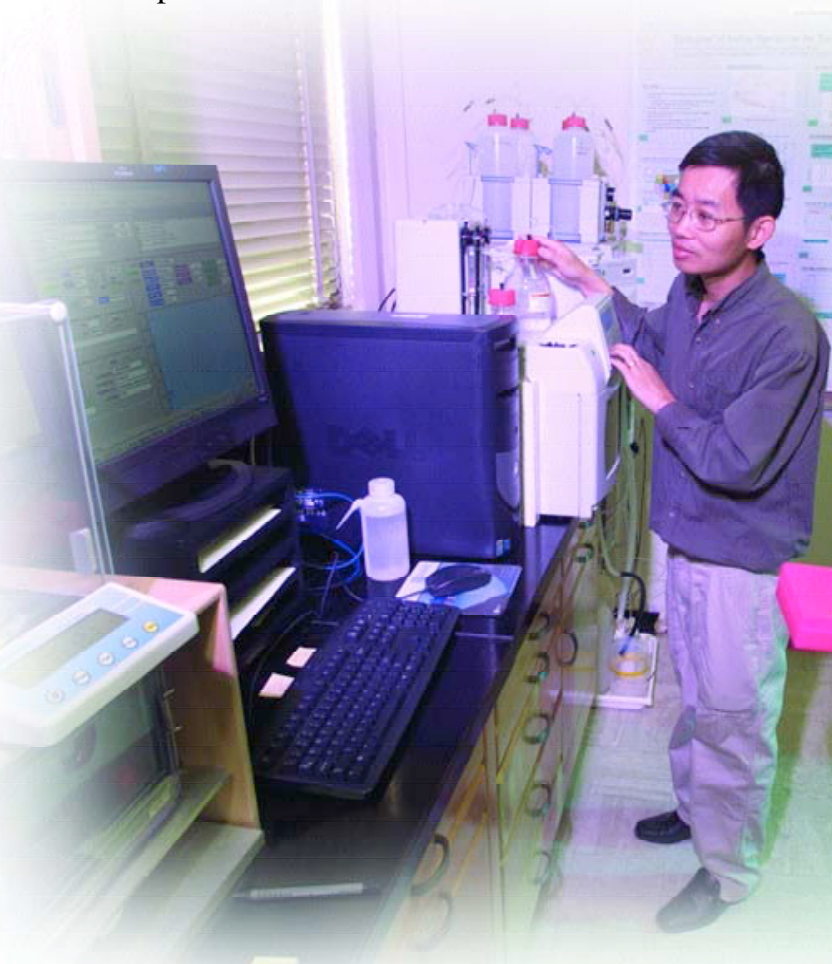
near-neighbor atomic bonding and
Examination of iodine species, with among species, is essential in interpreting the behavior of lodine in the environment. Using results from bench-top soil colum in various chemical form Specifict we focus on the sorption and transport behavior of iodine species (iodide, iodate, and 4-iodoaniline). In some clay minerals, we observed appreciable iodate reduction to iodide, probably mediated by the structural iron (Figure 1). We interpreted the sorption and transport behavior of different iodine species with respect to the phsicoical media which exhibit a wide geological mellia, which exhibit a wide soil $\mathrm{pH}$, and texture. We continue to develop new analytica methods for analyzing inorganic iodine forms and ${ }^{129} \mathrm{I}$ in complex environmental matrices. Employing a new multicollector inductively coupled mass
spectrometer (IsoProbe MC-ICPMS), we 作 sensitive technique of accelerator mass
spectrometry, the method has advantages both in ease of sample prepas advantages (allowing higher throughut) and in potential addition of speciation information

Scientific Impact Although nuclear fuel reprocessing the environment, trace levels are found nature. Local oxidation/reduction coused ans and biological activity have chemical equilibrium with naturally occurring ${ }^{129}$ I. Experimental studies allow us to predict the likely chemical form of 129I from fuel reprocessing emissions and to interpret the ${ }^{129}$ I to ${ }^{127}$ I ratios in downstream or downwind matrices with respect to the source, mode of transport and mechanism of incorporation. study suggests that soils with significant
JEAN MORAN (925) 423-1478, moran10@llnl.gov

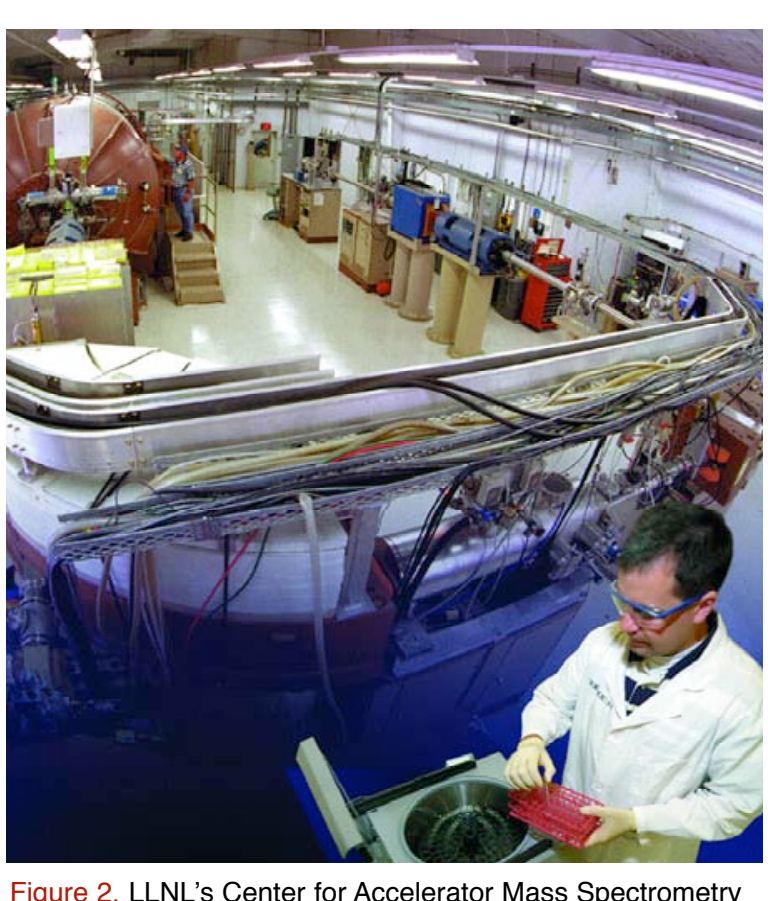

Figure 2. LLNL's Center for Accelerator Mass Spectrometry ingerprints of nuclear reprocessing emission. organic matter will sequester ${ }^{129} \mathrm{I}$ eficiently, and aqueous matrices such as 129I that originates in the atve to input of terms of dose estimates, our results indicate that atmospheric transport and inhalation may be a more significant pathway than previously thought, and 120 remains out of equilibrium with stable odine in the environment for up to

Related Publication QHu and J.E. Moran, "Simultaneous Analyses and Thacers in Hydrologic Sudies" Hydol Process, in press.

Q. Hu, et al., "Sorption and Transport of Iodine Species in Sediments from the Savannah River Hanford Sites," J. Contam. Hydrol, in press K. Schwer, et al., "Near-Conservative Behavior of Iodine-129 in the Orange County Aquifer Syst
NEW FRONTIERS

The ability to determine iodine peciation, coupled with major Center for Accelerator Mass Spectrometry (CAMS), is opening new field in tracer technology (Figure 2). Because CAMS requires only a small sample and can perform extremely sensitive measurements, fuel reprocessing emission fingerprints can be detected at greater distances long after the emission. Using 129 I tracer in fuel reprocessing emissions provides an advantage over the traditionally used ${ }^{85} \mathrm{Kr}$ because as a noble gas, $85 \mathrm{Kr}$ disperses rapidly in the atmosphere without a trace. The next advance will be to apply speciation information derived from these experiments and measurements of 129 fuel reprocessing emissions. 


\section{Age-Dating Groundwater to Assess Contamination}

2 uantitative characterization of contamination by conducting focused contaminants in groundwater. last time the water was in contact with Livermore is one of only two

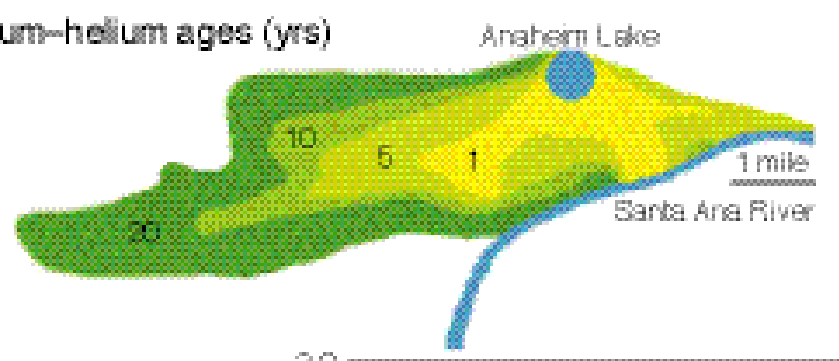
as recharge) and flow paths is essential to water resource management and environmental remediation. In response a request for assistance from California's We Resons 16,000 municipal water wells to studies on the nitrate and emerging Both radioactive and stable isotopes can provide information about groundwater's age, which refers to the the atmosphe re. Younger water is more requires close monitoring. facilities in the world with highthroughput capability for age-dating
analyses and is unique in using isotopic tracers to characterize groundwater travel times and flow path.

Tritum-helium ages (yrs)

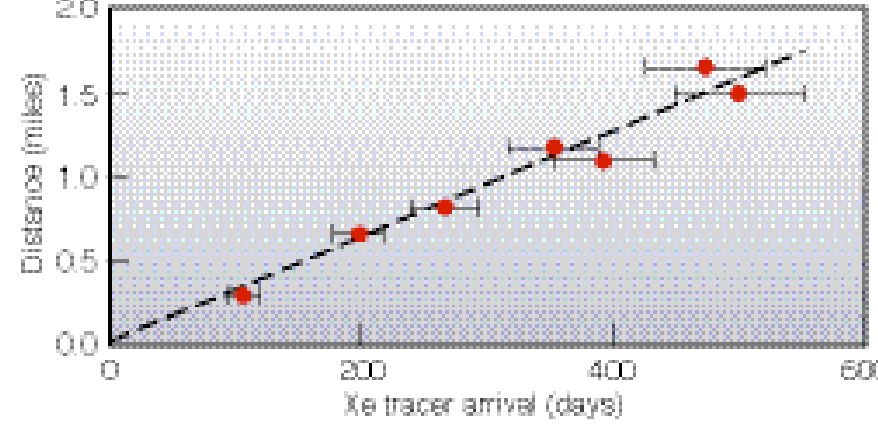

Figure 1. Groundwater age and travel distances are shown for a recharge facility in

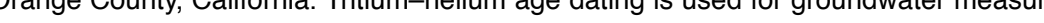

Relevance to CMS Research Themes Mass spectrometry using noble gases, developed at LLNL for nuclear test diagnostics. Over the years, its applicatio have been extensive in research for our is anso sources and transport in the ground at the Nevada Test Site.

These programs are closely aligned waste cleanup. Noble gas methods are also used in other research, including studies on groundwater flow and pathogen xenobiotics during land application of wastewater.

Major Accomplishments in 2004 Our unique approach uses noble gas flow through tritium-helium age dating issues thission to tackle environmenta biogeochemistry, and transport of and xenon tracer studies (Figure 1). Tritium-helium age dating measures groundwater in units of years, and (recers measure groundwater with ages in days. One drawback of using age to asse vinerabity is that old groundwater young water that is highly conteminted. To address this possibility, LLNL, in collaboration with the United States Geological Survey (USGS), also measure recently released volatile organic compounds such as MTBE (methyl tertbutyl ether) at very low levels. We have found hat coastal aquifers, particularly in the Los Angeles Basin and extensive low-permeability zones as barriers to vertical contaminant transport (Figure 2). Although their young water shows low levels of common organic contaminants, their old (deep) water is free of anthropogenic contaminants. The absence of contamination in deeper groundwater is remarkable considering that these basins are located in some of the most highly compromised areas in the state We have found the reverse situation fo California's Central Valley, especially northern California where the principal aquifers are not well protected. Common solvent contaminants are present in both young and old groundwater in the Sacramento Valley, a distribution requiring more than one transport path for is rapidly developing, and Central Valley is rapidly developing, and significant contamination from affecting the drinking

Scientific Impact

The ability to characterize groundwater flow is essential to understanding contaminant and pathogen transport. We have developed a new method to calibrate and validate high-resolution simulations groundwater flow and con minant
For more information contact

BRAD ESSER (925) 422-5247, esser1 @ llnl.gov ages for large numbers of samples. By using noble gas mass spectrometry, w have characterized the flow of water within a basin to determine the spatia distribution of groundwater age. We are aso using noble gas methods to determine of dissolved gases above the equilibriun solubility limit in water. These parametes are directly related to the elevation,
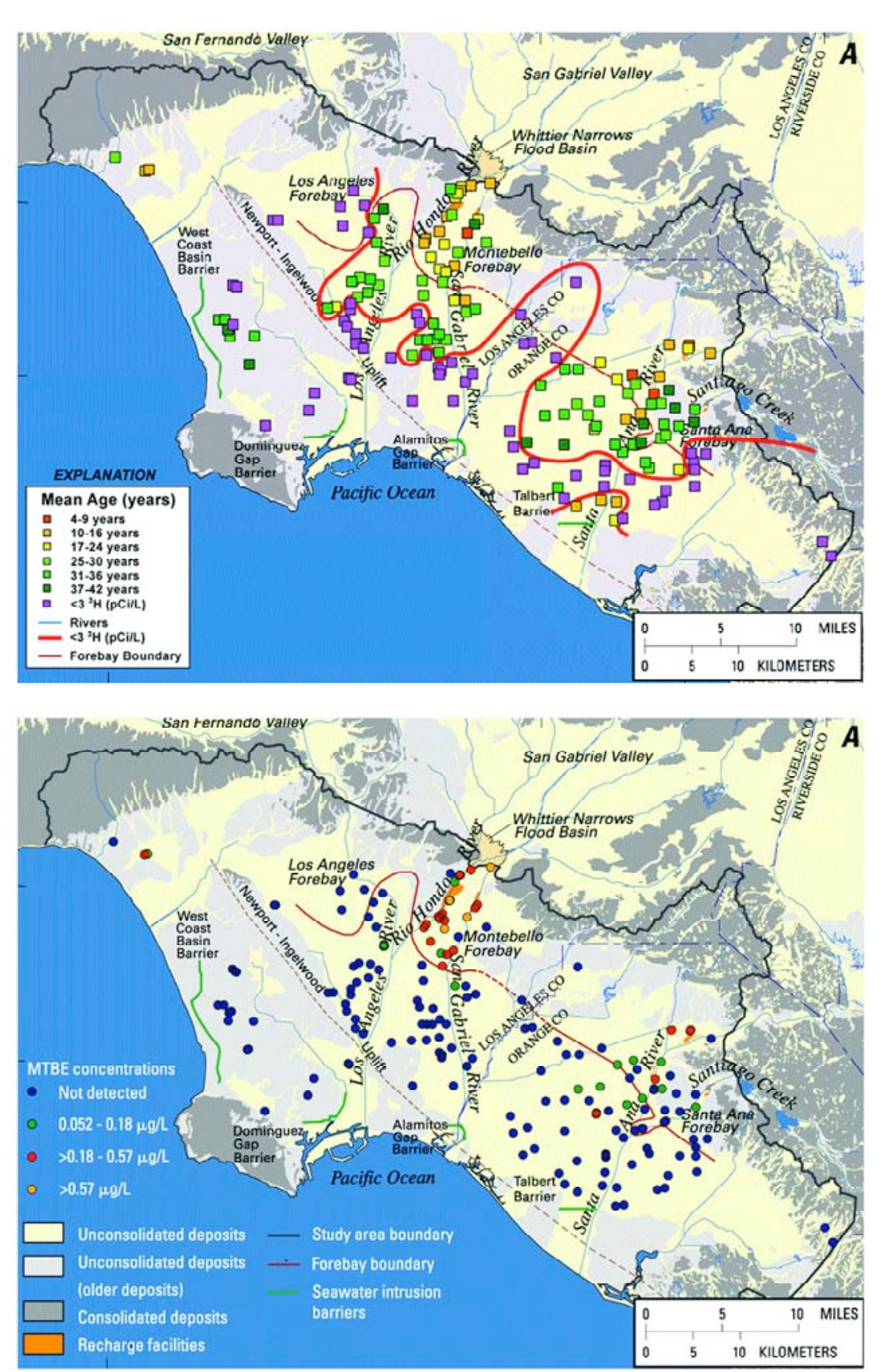

seasonality, and soil conditions during Related Publications California," Ground Water 42(2). 167-174 (2004) lange data sel has revealed strong regional Basin," Lawrence Livermore National Laboratory ditferences in water recharge that have not J.F. Clark, et al,. "Geochemical Imaging of Flow near cas_Inn_sac pder)L.K. Rademacher, J.F. Clark, and G.B. Hudson, Spring Waters: Implications for Recent Changes in Climate and Atmospheric Circulation," Geology $30(2)$ $139-142$ (2002)

\section{NEW FRONTIERS}

Because most anthropogenic groundwater contamination has research using age dating and tracer studies has been useful for assessing the vulnerability of groundwater to contamination. The next step will be develop new tools to characterize components in younger groundwater. nuclear reprocessing, has a similar hat life to tritium, but a very different source function. Because the activity of ${ }^{85} \mathrm{Kr}$ in of very young groundwater (less than 10 years old), we are planning to develop a low-level method that uses ${ }^{85} \mathrm{Kr}$ for water resource investigations. We are also continuing our research to determine how noble gases are incorporated into infiltrating groundwaters, during both natural and artificial recharge, as well as the use of isolopically enriched noble gases as

Figure 2. A map of the Los Angeles Basin shows the levels or the organic contaminant MTBE tound in groundwater. MTBE is found only in shallow, young groundwater, demonstrating hial deep grondwater protected from surface
courtesy of the USGS. 


\section{Isotopic Composition of Interplanetary Dust Particles}

nterplanetary dust particles (IDPs) are extremely small grains derived from primitive solar-system bodies such as
asteroids or comets. These dust grains asteroids or comets. These dust grain carry traces of isotopes that may have originated in stars older than our solar system. Laboratory observations of these particles at he nanoscale can advance our
understanding of the origin and formation
of solar system and galactic processes. For example, IDPs that contain submicrometer grains with nonsolar isotopic composition can be confirmed originating from interstellar space
(Figure 1). The NanoSIMS, a new(Figure 1). The NanoSIMS, a new-

our researchers a unique capability to measure isotopic composition in IDPs (Figure 2). NanoSIMS is also used in other cosmochemistry projects, including
the study of the abundances of short-lived the study of the abun
nuclides in meteorites.

Relevance to CMS Research Themes Identifying and characterizing IDPs a
the nanoscale is part of a larger effort tha utilizes an array of microanalytical techniques for materials characterizatio In addition to furthering our understanding of astrophysics and cosmochemistry, this research co to our national-security mission.
Determining the origin of samples with

unknown provenance based on chemical and isotopic signatures applies not only to investigations of meteorites, interplanetary dust, and samples returned from planetary

Majop Accomplishments in 2004 In collaboration with the Institute of (a) NanoSIMS to identify and report the firs discovery of nitrogen isotopic anomalies in a hydrous IDP (Figure 3). In contrast to anhydrous IDPs that are most likely from cometary origns, hydrous IDPs are

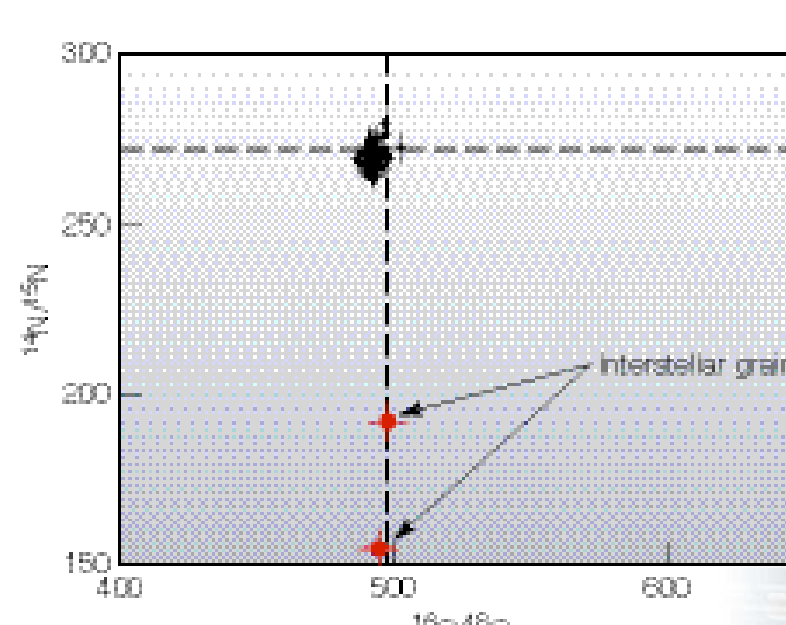

Figure 1. An isotope-ratio plot of nitrogen and oxygen shows the composition of an interplanetary dust particle. The two red circles represent interstellar grans identhed with the IDP. The carbon interstellar grain has a $14 N / 5 \mathrm{~N}$ ratio of 272. The black circles represent remaining areas of the interplanetary dust particle that have a solar isotopic composition.

Figure 2. The NanoSIMS, a newgeneration ion microprobe, was used to report the first discovery of nitrogen
isotopic anomalies in a hydrous IDP.

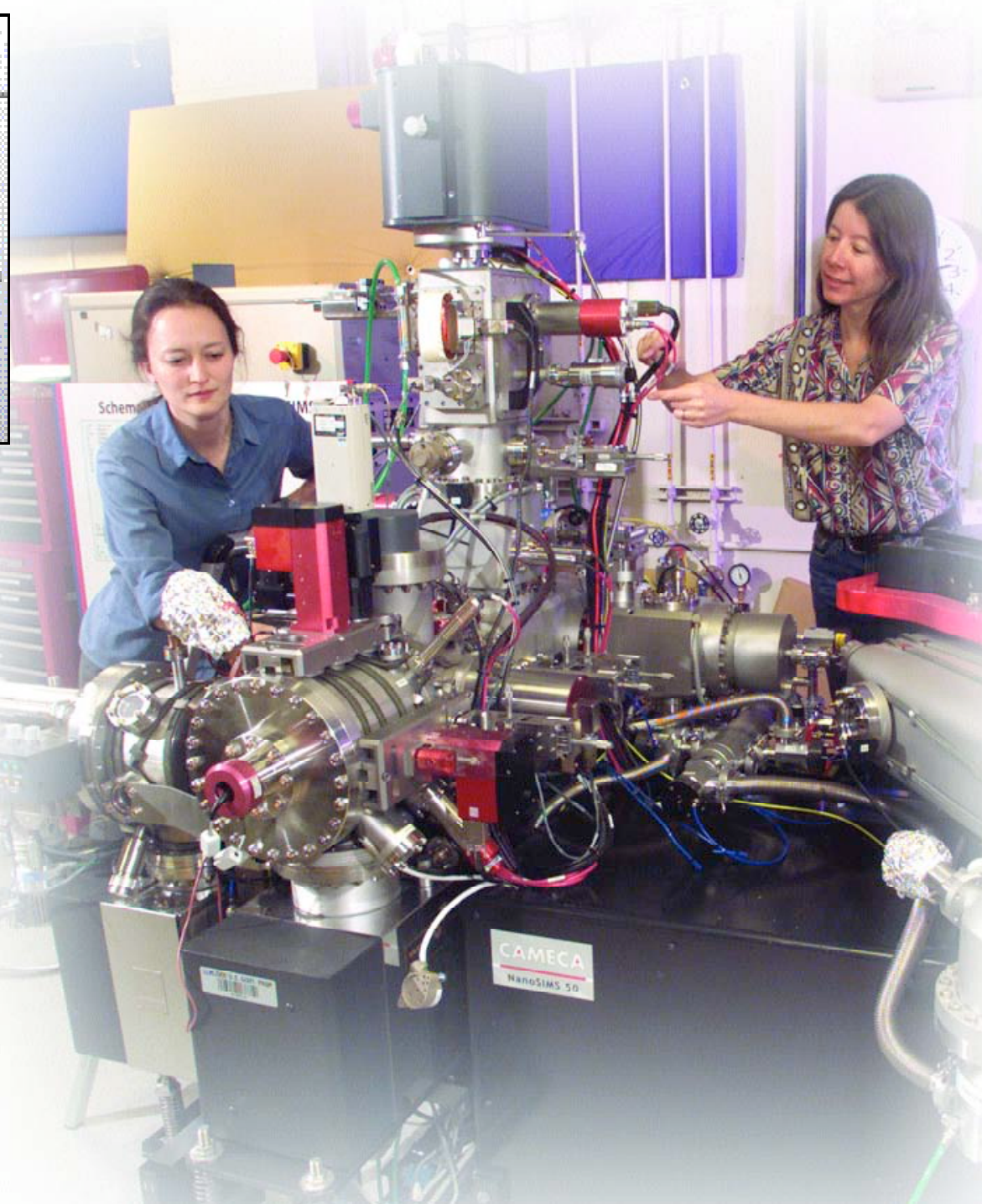

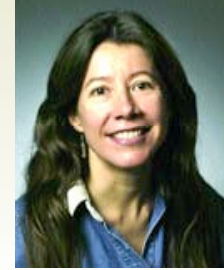

For more information contact JULIE B. SMITH (925) 422-9607, smith371@llnl.gov
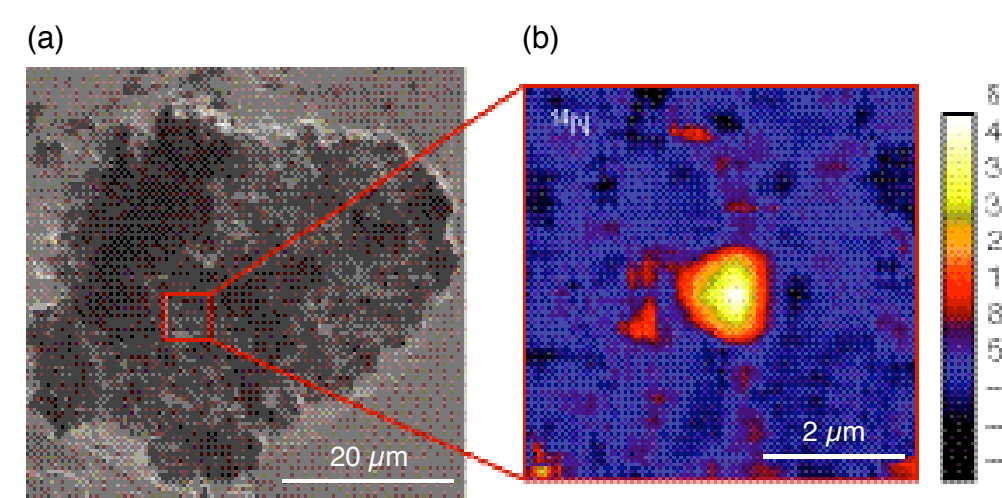

Figure 3. Images from NanoSIMS show (a) an interplanetary dust particle, (b) nitrogen-isotope-ratio-ion image of a carbon interstellar grain within the particle an (c) an image of the section of the particle containing the carbon grain removed by a

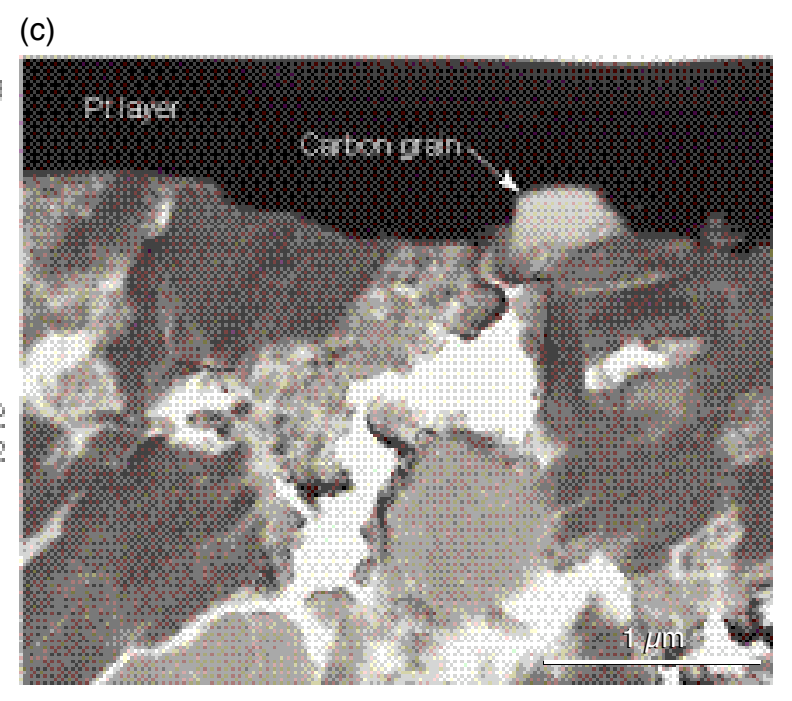

preservation of nitrogen isotope anomalies in submicrometer amorphous carbo information on the temperovides duration of aqueous alteration in the asteroidal parent body.

Preparing samples from bulk material is a critical step in nanoanalytical studies. Advances in the use of the focused ion beam (FB) to ablate and isolate grains he Using microscope, we can determine the composition of a single slice of a grain taken from the FIB.

Using a new-generation, scanning transmission electron microscope fitted with a high-resolution electron energyloss spectrometer, we can identify the sources of spectral signatures observed have identified the possible origins of astronomical feature - known as the 2175-angstrom extinction featureis the strongest spectral signature of interstellar dust. Our research has identified two likely sources of the 2175-angstrom feature-organic carbon interstellar medium.

Scientific Impac

Because much of what is known about our solar system comes from comparing spectral studies of space with laboratory suces of in is essent to fin specifically, the presence of both organic carbon and amorphous silicates may help explain the variability of the 2175-angstrom feature, the relative abundances or physical state the carriers can be expected to vary from one line or sight to another. In addition to the study of IDPs, we are also conducting studies of presolar silicon carbide grains hortlived radionuclides on

Related Publications

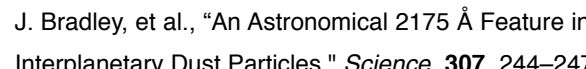
(2005). and amorphous silicates-both of whic
are abundant in both IDPs and in the
AN. Krot, et al. "Chronology of the Early Solar Rich Inclusions," Nature, 434, 998-1001 (2005).

M. Wainwright, et al., "Studies on Bacteria-like Aeorobiologia, 20, 237-240 (2004).

By combining microanalytical echnologies such as multibeam FIB, NanoSIMS, and our recently installed SuperSTEM), we are at the forefront of nanoscale characterization. A direct applcallon or our IDP studies will be to analyze samples from NASA's
STARDUST mission, which is scheduled dust from the comet Wild 2 We will study the comet particles to learn more about the fundamental materials that assembled to form the solar system 4.6 billion years ago. System from Chondrule-Bearing Calcium-Aluminum-

NEW FRONTIERS high-resolution scanning transmission 

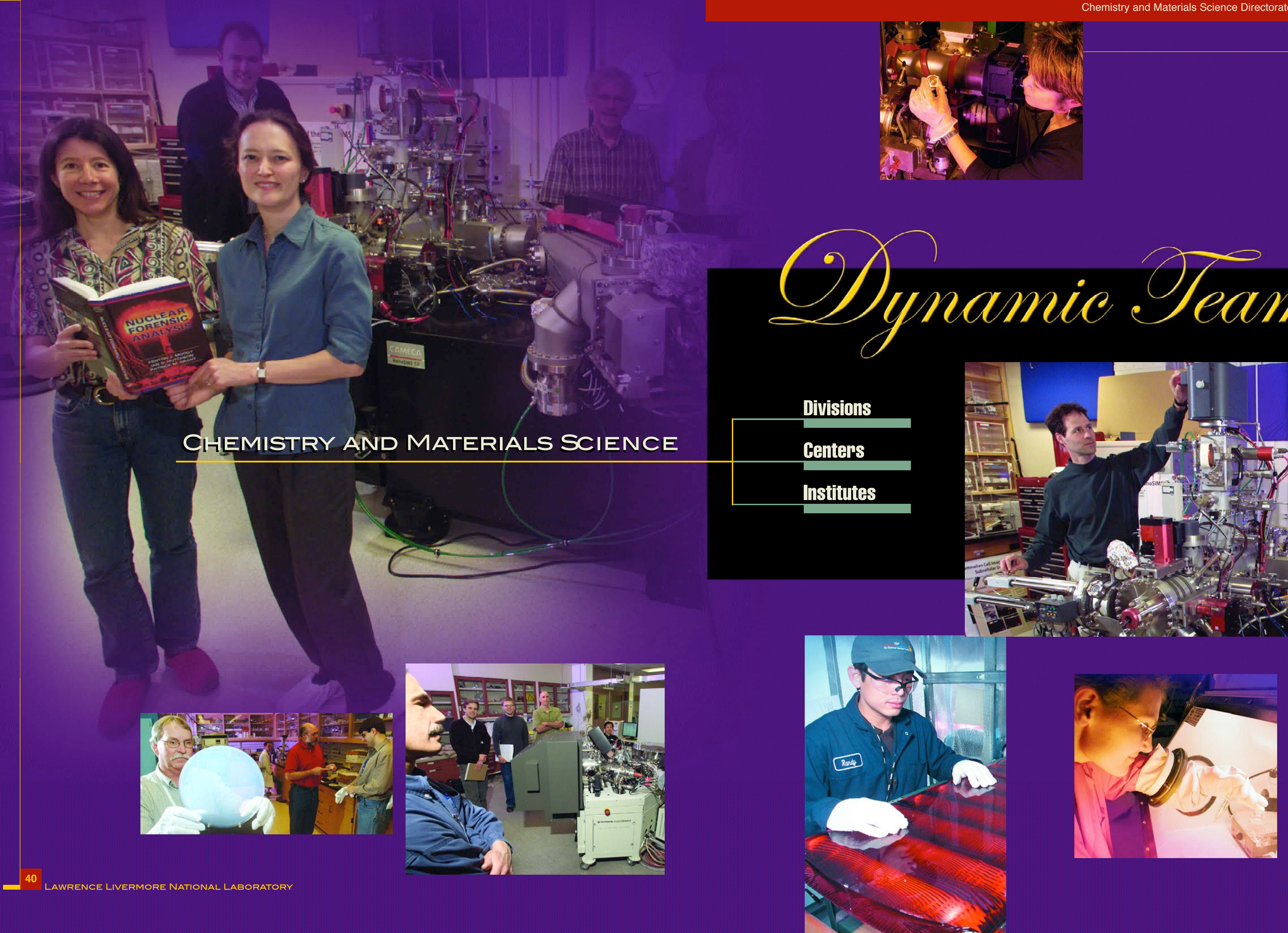


\section{Materials Science and Technology Division}

Providing a fundamental scientific capability for predicting the properties and performance of materials under extreme conditions

7 he Materials Science and Technology Division (MSTD) executes world-class R\&D in a wide range of disciplines. Our program elements are aligned with specific projects supporting the U.S. Stockpile Stewardship Program, the National Ignition Facility (NIF), the Yucca Mountain Project, and the nation's counterproliferation efforts. We are pursuing conventional-scale and nanoscale technologies in areas th made metaris characterization, high-performance computational materials science, electrochemistry, ceramics, solid-state physics and chemistry, surface and interface science, ultrafast science, and coatings and multilayered materials.

\section{Strategic Theme-Related} MSTD is aligs in the CMS search theme of materials properties ar performance under extreme conditions. The division supports the Nanoscale Synthesis and Characterization Laboratory (NSCL), and many of our research efforts are described in that section of this repor. The following are highlights of addition MSTD accomplishments in 2004

Materials Dynamics: We continue to employ multiple approaches-includin recovery-based observations, and largescale computational modeling - to better understand the dynamic response of materials under high-pressure, rapid shockloading conditions. For example, to study shock propagation and phase transition of titanium and vanadium under shock loading, we are using extended $x$-ray
absorption fine structure measurements the nanosecond time scale.

Our studies of material deformation rate loading conditions on a laser-based system. Experimental studies of shockinduced melting and refreeze-on-release phenomena are beginning to address complex issues of microstructure formation under highly transient conditions. The division's non-equilibriu molecular dynamics models are examining defect production and and have provided extraordinany demen material response to shock-wave loading in single crystals and polycrystalline metals (Figure 1).

Ultrafast Science-Dynamic Transmission Electron Microscope: In addition to $x$-ray and optical techniques, we are using electrons as an ultraf probe. The transmission electron spatial resolution, allows us to sudy defects in materials that often control

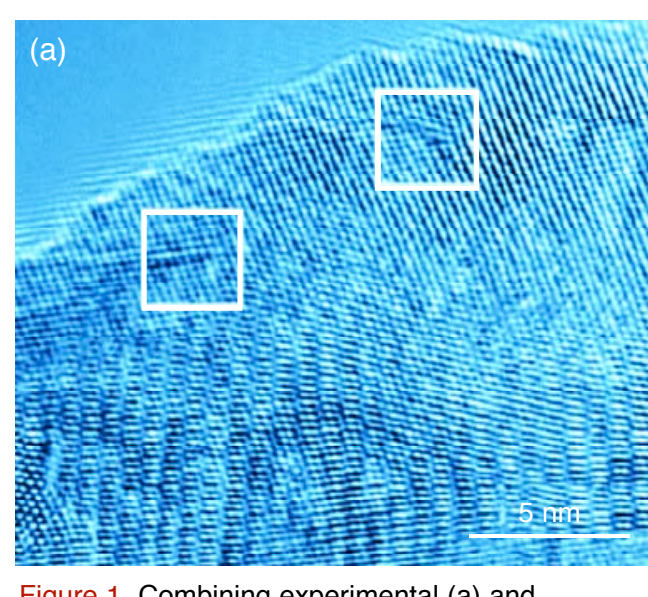
Figure 1. Combining experimental (a) and computational (b) techniques, we can observe dislocation in nanocrystals and polycrystals a grain sizes less hin $12 \mathrm{~mm}$. Boxes denote material behavior. We are constructing a dynamic transmission electron microscop (DTEM), which will capture

microstructural dynamics at nanosecond and nanometer scales, allowing us to study the mechanisms by which defects transform materials from one state to (File 2). Our DTEM will use an electron-capable digital camera system in place of he film traditionally used in a conventlynal TEM. The images we are understand pefome necessary for future single-shot investigations. As shown in Figure 3, the pulses produced by a DTEM are intense enough that an image can be observed directly on a phosphor screen

Computational Materials Science: We have efforts under way-including the massively parallel ParaDis code covered effects of adis report to stady the dislocation dynamics on the mechaici performance of a wide variety of

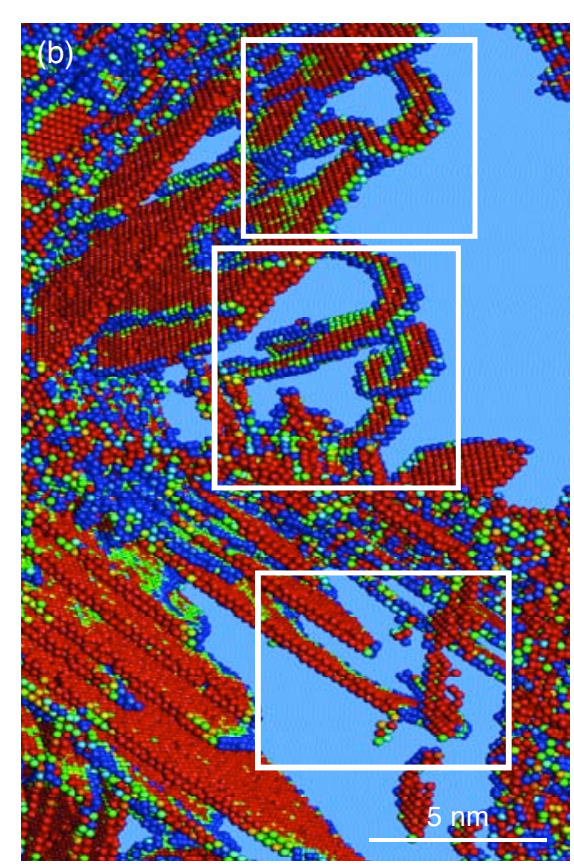

For more information contact

materials. Particularly, we employ density-functional theories in our model to understand the nature of the electronic properties of the six solid phases of plutonium found at ambient pressure. This year, we have extended a model to inclu

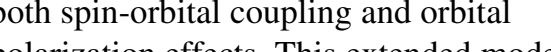
as been able, for the very first time, to accurately simulate the total energies, crystal structures, atomic densities, and magnetic moments of plutonium.

Radiation Detectors: We continue to provide novel materials innovations to national-security programs. We have and fabris de onstrated the synthesis an be deployed and interrogated remotely.

Using fluorescent photochromic materials, we have developed radiation dosimeters that are small and inexpensive to produce. As radiation converts hotochromic molecules in the sensor

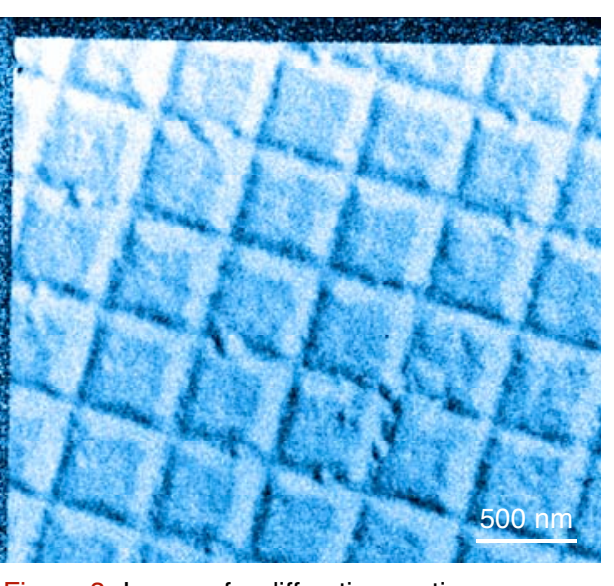

Figure 3. Image of a diffraction grating produced
operation.

fluorescers, a laser system can interrogate the fluorescence level remotely to $d$
radiation dosage. We have already demonstrated functional high-sensitivity dosimeters and will continue optimization this year. This technology is already making contributions to the nation's

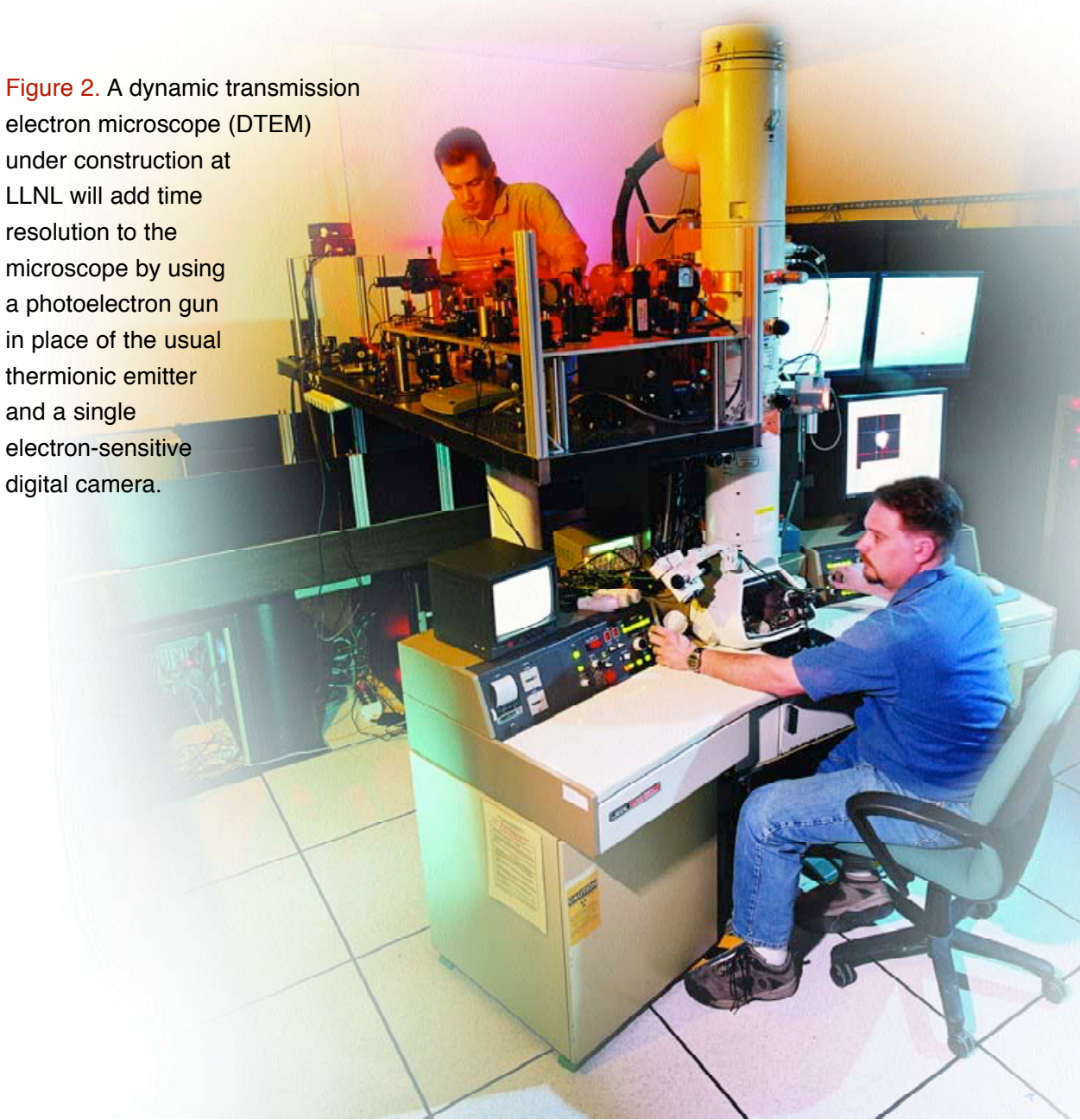

ate-of-the-Art Technologies 政 extreme conditions. Our focus has be on the following scientific areas: ayns hesis of high-performance materials for NIF targets, multilayer coatings for extreme-ultraviolet lithography, and thin films for lowemperature welding - Laser-matter coupling and dynamic response of materials, wi particular applications to NIF experiments and stockpile stewardship Long-term corrosion studies to nuclear waste repositories - Experimental facilities for the study of dynamic properties of materials at unprecedented spatial and temporal resolution - New materials for remotely interrogated radiation detection

\section{NEW FRONTIERS}

MSTD will continue to develop facilities with massively parallel computing to investigate the properties and performance of materials under extreme conditions. A particular focus wil be to expand our capabillies in the area of ultrafast science including microscopy and diffraction.

We expect that the properties of new materials developed using ou nnovative materials synthesis approaches will be useful to many Thel and unexpected application experimental facilities will also help attract leading university collaborators and outstanding youngs 


\section{Chemistry and Chemical Engineering Division}

Science and engineering that investigate the chemical properties, reactivity, and synthesis of energetic and optical materials

7 he Chemistry and Chemical Engineering Division (CChED) focuses on discipline-based science and advanced materials synthesis, and chemical engineering while simultaneously providing key support to the Laboratory's programs in the areas of energetic materials, optical materials, an chemical detectio

\section{Strategic Theme-Related}

CChED is aligned with the CMS esearch theme of chemistry under extreme conditions and chemical engineering in support of nation security programs. Chemistry under extreme conditions involves processes at

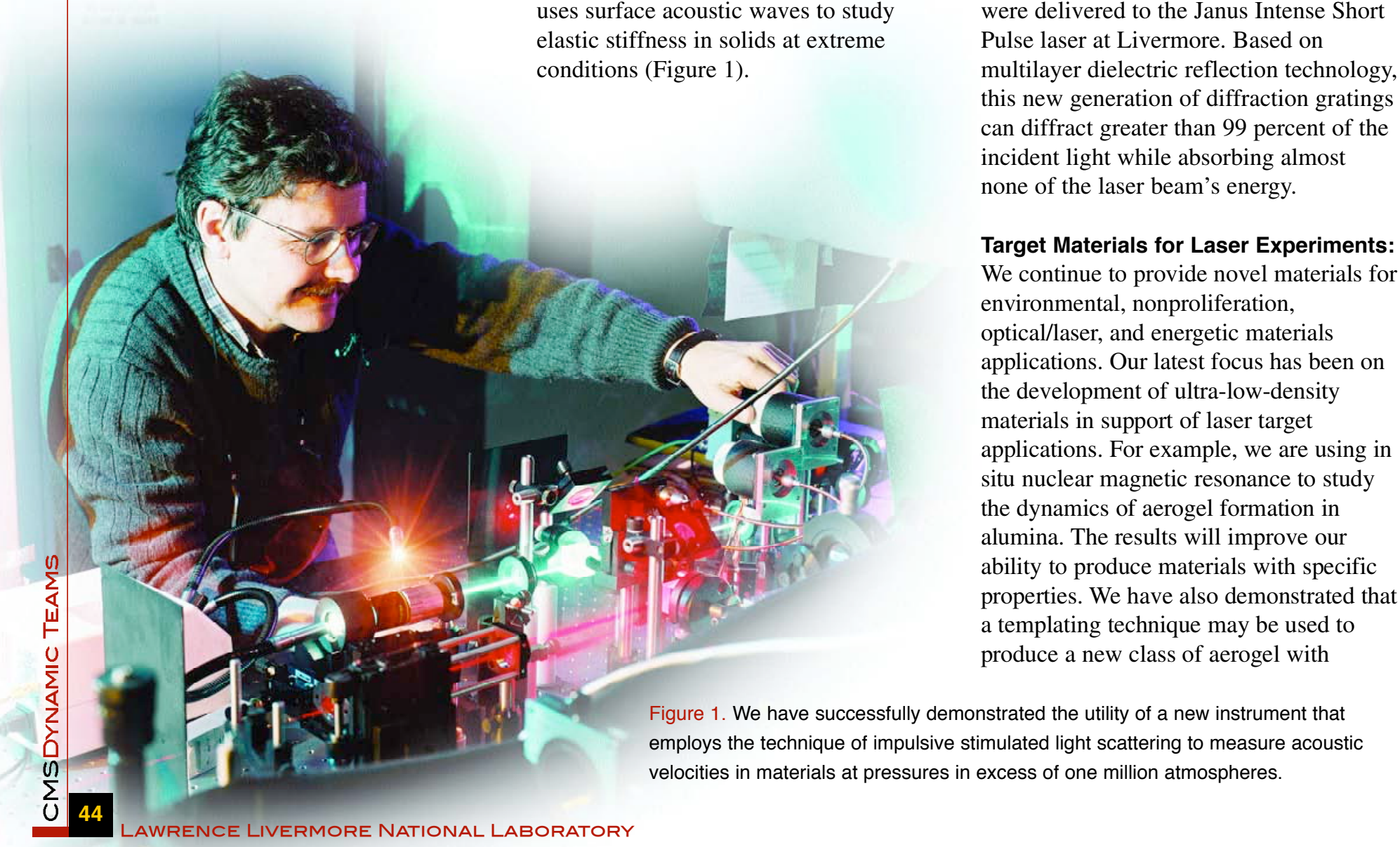

very high pressures and temperatures- $\quad$ Optical Materials for Laser coper to the optics for NIF, the world's largest lase Facility (NIF), and planetary interiors. Many of the division's recent (potassium dihydrogen phosphate) accomplishments are reflected elsewhere crystals, as seen in Figure 2. We have also in this annual report. The following are developed the first disposable debrisadditional noteworthy accomplishments shield optics for the NIF Early Light by CChED personnel in 2004: target chamber, which will greatly reduc Chemistry Under Extreme Conditions: contamination-induced damage to the We are using both experiments and of technologies for producing the world's gratings, which are used in petawatt ock waves. We have developed a new impulsive imulated light scattering instrument gratings, which are used in petawatt laser to allow for safe amplification of laser beams. Recently, the first-ever 80-cmaperture production gratings (Figure 3) this new generation of diffraction grating chent (S) example, we are using in formation in 作 moperties. We have also demonstrated tha produce a new class of aerogel with

\section{8}

For more information contac RANDY SIMPSON (925) 423-0379, simpson5@llnl.gov

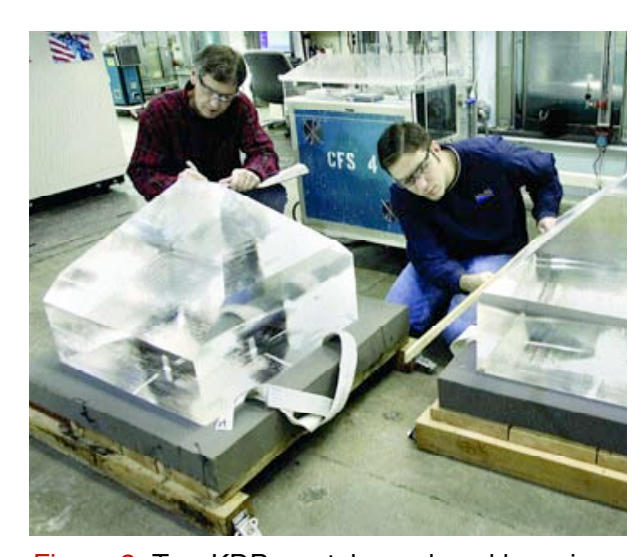

Figure 2. Two KDP crystals produced by using

densities less than $100 \mathrm{mg} / \mathrm{cc}$. By applying extran pyrolytic templating, we have 4 percent full density, which represens ignificant advance in this field.

Computational Chemistry: Our computational chemists have made noteworthy advances in a variety of projects, including first-principles studies water, with an emphasis on the poten energy surfaces of multiple water Stockpile Stewardship Program our studies have shed light on the effects of water on the interfacial structure and dynamics of the composite materials silica and polydimethylsiloxane in our aging nuclear stockpile. This work helps expl how dehydration might affect polymer stiffness in nuclear weapons stiffening Wer time and under very arid condition. We have also used theoretical calculation (1,35-tri-amino-2, 6 -trinitro benzene, a commonly used energetic material in nuclear weapons) at pressures from atmospheric to $7 \mathrm{GPa}$, reproducing observed highly anisotropic behavior and suggesting explanations based on structural features of the molecule. In other computational chemistry efforts, our group received a prestigiou Engineers for its work explaing how

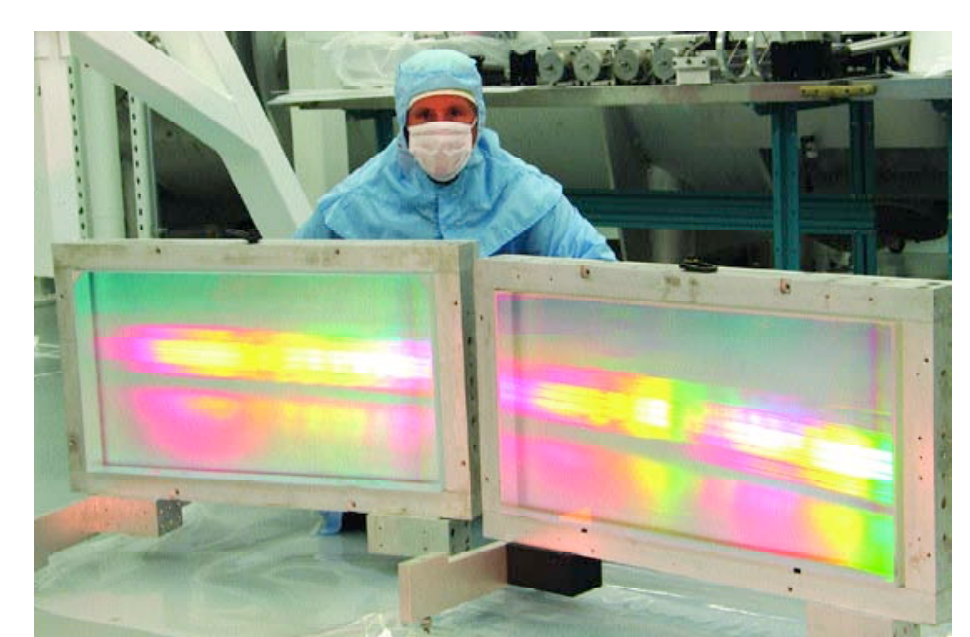

Figure 3. These firstever 80-cm-aperture production gratings
are composed of multiliayer dielectric reflectors. addition of oxygenated hydrocarbon

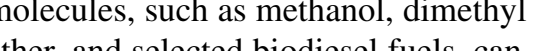
reduce soot emissions from diesel engines. The oxygenated species encourage the production of carbon monoxide and reduce production of significant soot precursors such as acetylene and butadienes. These results also explain a number of other observations about soot production in engines and the combustion of aged

\section{State-of-the-Art Technologies} CChED continues to develop new optics manufacturing techniques for NIF.
Better understanding of the crystallizatio and growth process has led to improved performance with lower levels of crystal growth defects, reducing bulk damage. Our expertise is helping researchers sol-gel coatings to design effective mitigation schemes.

Through a new collaboration with Lawrence Berkeley National Laboratory, we are using a high-pressure and temperature $x$-ray synchrotron beam line at the Advanced Light Source to monitor chemical reactions in the diamond anvil cell. In one of the first experiments, we Other work includes using in inorus. characterize mechanisms of surface heated diamond anvil cell that can acheve a pressure of $10 \mathrm{GPa}$ and a wide range of high-pressure reaction and materials properties.

\section{NEW FRONTIERS}

Through several research collaborations, we are working to advance our understanding of energetic ming combines experimental studies an theoretical calculations, we can more fully comprehend dynamic chemical pressures.

We must use our expertise and advanced tools to fully understand the fundamental issues that govern the safety and performance of our national processes, understand solid-state nanoparticle chemistry, and augment homeland security agendas. For example, there is a tremendous scientific opportunity to conduct pioneering scientific work in fundamental chemistry at pressures above $1 \mathrm{kbar}$ that has direct applications to the processes at high temperatures and Laboratory's core mission and to address outslanding questions in 


\section{Chemical Biology and Nuclear Science Division}

To provide unique and integrated solutions to issues at the intersection of chemical, biological, and nuclear science and to sustain a strong and viable life-sciences expertise

7 he Chemical Biology and Nuclear Science Division (CBND) sup program areas that include the
Stockpile Stewardship Program homeland security, counterproliferation, and energy and environment. The division has exceptional expertise in radiochemistry, radiation detection, nuclear sciences, groundwater chemistry, biochemistry, and bioanalytical science. CBND also provides a majority of the technical staff in CMS institutes and
centers, including the Glenn T. Seaborg centers, including the Glenn $T$,
Institute, the BioSecurity and Nanosciences Laboratory, and the Forensics Science Center.

Strategic Theme-Related CBND is aligned with 4 CMS esearch themes of science in support of national objectives at the intersection of chemistry, materials science, and biolog as well as in applied nuclear science for human health and national security. The following are highlights of CBND accomplishments in 2004:

Biological Trace Particle Detection: beveloped by Livermore scientists to Spectrometry (BAMS) systmass spore species in less than one minute. In

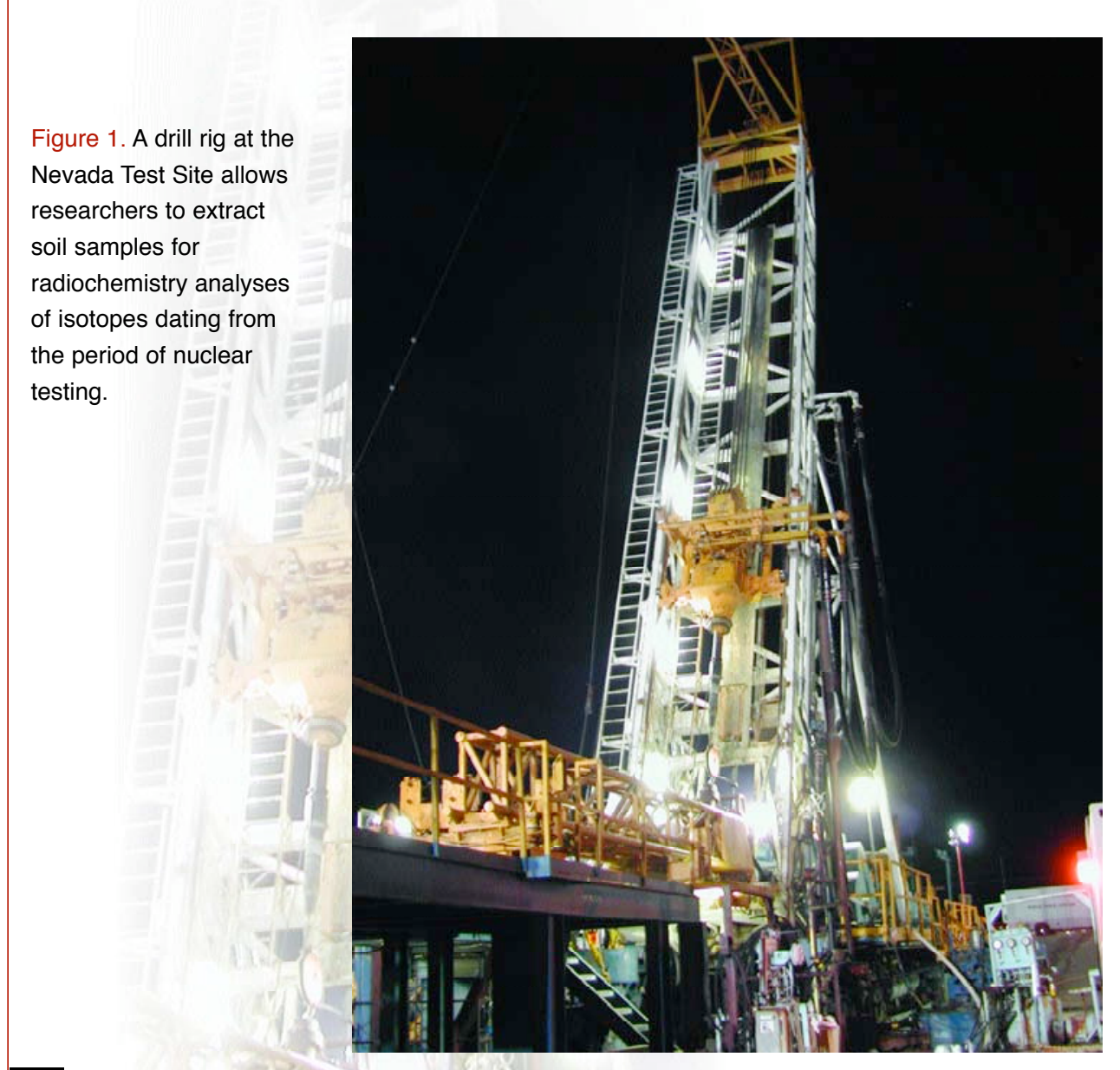

46
2004, we made significant improvements that will increase throughput by several limits for trace populations of particles. We have improved the particle tracking system by increasing the number of probes and analyzing lasers from two to six. We have also developed an advanced algorithm on a field programmable gate array, which can process data in real time. This year, we successfully participated in several government-run field capability tests and spectrometer sensitivity limits.

New Radioanalytical Tools: We continue to provide analyses on environmental and hazardous waste samples that cannot otherwise be analyzed at commercial facilities. These analyses are certified by
the State of California and qualified by the State of California and qualified by
the Department of Energy Environmental the Department of Energy Environ Management Consolidated Audit
Program. A major effort this past year has Program. A major effort this past year has
been to support a $\$ 20$ million project to downgrade the hazard category of the Heavy Element Facility at LLNL from a nuclear facility to a radiological facility, by providing gamma spectroscopy and radioanalytical support to identify and remove items with high activities of

exotic actinide radionuclides.

During the period of underground

nuclear weapons testing, CBND

performance by extracting debris, known as drillbacks, from the soil surrounding a test and using radiochemistry for diagnostics. Because drillbacks were debris selection criteria were based on isotopes with rather short half-lives. In 2004 , we led a project that drilled back soil samples near a previous nuclear test on a new set of radioactive decay sequences, establishing new

radioanalytical tools for the future (Figure 1). In addition, isotope analyses melt glass and groundwater samples fro the drillback sanples have revealed tow raclid and liquid phases. These results been used to build predictive models of contaminant transport rates in groundwater, which are useful for the environmental management programs the Nevada Test Site for the Department of Energy.

Radiation Detectors: We have develope a new, innovative large-area gamma-ray structure that uses quantum confinement to provide tunable frequency output and improved signals. This class of new detectors will be used for national secu applications. We are also building a neutron detector with semiconductor nanowires embedded in a boron matrix. The length-to-diameter ratio of the silico nanowires is approximately 10.1 (Figure 2). These features will allow the be less costly to produce, and provide higher energy resolution than that of previous sensors. We are also pursuing a new approach to fabricate neutron detectors based on a single-crystal diamond fabricated by the chemical vapor deposition technique. The diamond neutron detector should exceed the performance of natural diamond gemstones, providing an excellent neutro Thermonuclear Experimental Re the National Ignition Facility. Other highlights of CBND activities 2004 include the development of an automated titration system to make corrosion studies more efficient and an innovative photocatalytic nanolithography technique to study pattern surfaces efficiently and contribute mation contac

A ROSENBERG (925) 424-5212, rosenberg4@ @llnl.gov
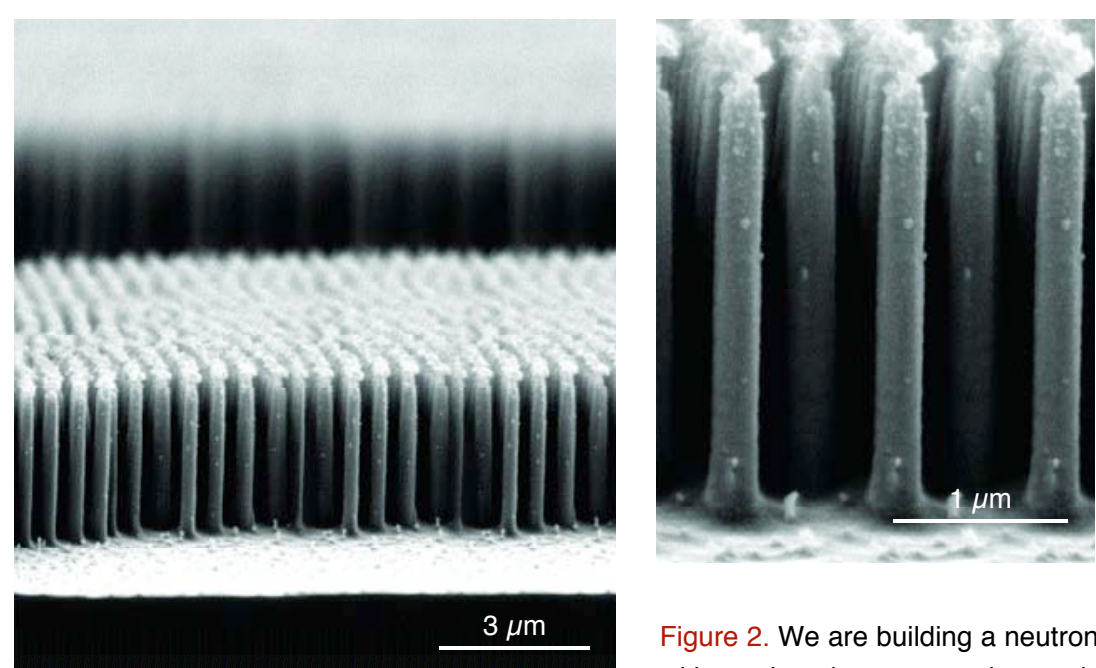

\section{$3 \mu \mathrm{m}$}

scientists also contributed to homeland security by leading a feasibility study for detection and identification of radioactive materials in cargo container in the New York City area.

State-of-the-Art Technologies CBND possesses unique expertise that is applied toward a wide range of analytical chemistry disciplines. For example, our support for the Departm capabilities in rediation imaging, nuclear attribution technologies, and air-sea-land port-of-entry protection. The skills and equipment used for radioanalytical studies of nuclear tests is now being used to produce and observe the radioactive decay of new elements 1 through 116.

NBNDis home to one of only te Spectrometer) in the lon Mass Spectrometer) in the world. The capabilities of nanometer-scale spatial resolution and part-per-million sensitivity, is being used in studies of biological materials, molecular targeting for cancer therapy, cosmochemistry, an particle characterization for forensic. Additional state-of-the-art analytica instruments are in regular use in our
laboratories.
Figure 2. We are building a neutron detector with semiconductor nanowires embedded micrographs show silcon nato approximaty 200 nm diamoper with $1: 05$ aspect ratio made by nanosphere lithography and deep reactive ion-etching technique.

\section{NEW FRONTIERS}

CBND provides expertise to many major LLNL programs, each with chemica bione goals. In the area of future program is the national

Genomics:GTL (formerly Genomes to Life), in which CBND plans to participate in both the basic research and facility initiatives.

In support of the U.S. Stockpil Stewardship Program, our radioanalytical study on drillback soils is expected to lead to important new advance methods on screening for to biological agents and high-explosives compounds in real-time field applications in support of homeland security. Many environmental programs are benefiting from the water R\&D initiatives led by CBND. With each of these major LLNL programs likely to grow in the coming years, CBND is well positioned to contribute leadership and chemical, and nuclear science. 


\section{Biosecurity and Nanosciences Labopatory}

Science to protect the nation against biological threats and natural disease outbreaks

The BioSecurity and Nanosciences Laboratory (BSNL) is a initiative at Lawrence Livermore. The BSNL mission is to provide the natio with biochemical and biomaterials expertise to detect and identify harmfu molecules that threaten human health an national security. Principal research are include proteomic analysis, bioaerosol synthesis, nanofabrication of device platforms, and cellul
scale measurements.

\section{Strategic Theme-Related} Accomplishments in 2004 BSNL scientists conduc multidisciplinary research to help our nation's

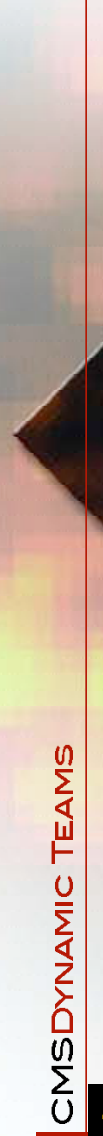
science, pathogen signatures, bio-orga

homeland security organizations counter molocular wapons, to understand the fight life-threatening diseases. Its focus is in alignment with the CMS research theme of science in support of nation objectives at the intersection of chemist materials science, and biology.

Pathomics is the study of changes in protein and metabolite expression levels in response to disease-causing agents, pcientists improved bS a hility to ides

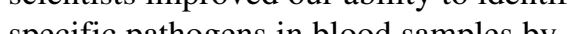
coupling liquid chromatography with

mass spectrometry, using protein samples from humans and from Yersinia pestis or plague bacteria. Our scientiss used separation with liquid the dynamic concentration range of the

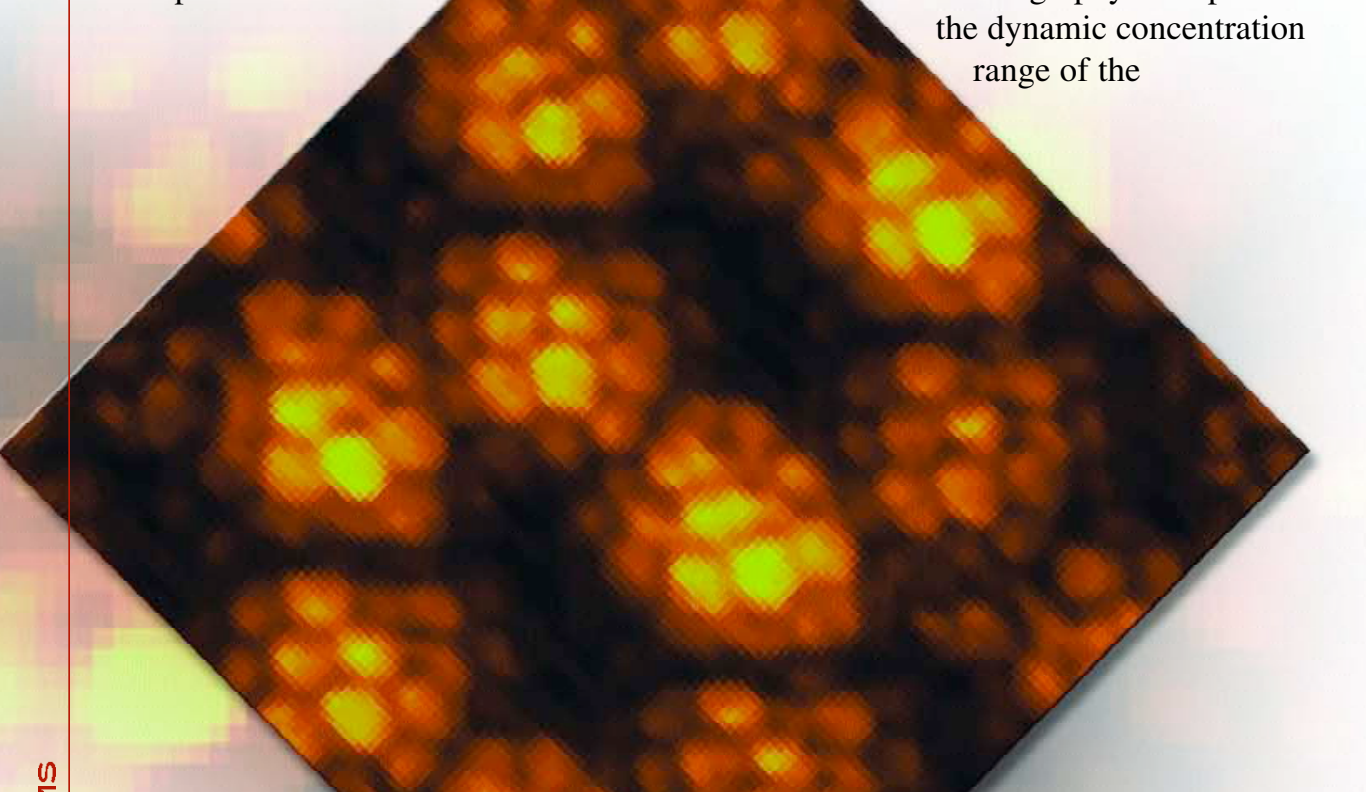

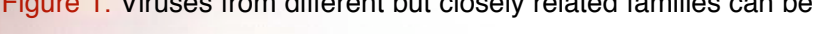
differentiated on the basis of their capsid structure (the protein shell of a virus). This image, taken by our atomic force microscope, of a crystalline at ultrahigh resolution.

spectrometer, identify unique peptides produced from each protein, and produce Identifying human pathogens and characterizing their surfaces is critical in understanding how pathogens cause disease and in developing detectors and vaccines for biodefense and medical applications. BSNL scientists continue to push the limits of atomic force proteins and spores. Duaging individual approached a 2-nm iesulg 204, we the structure of a small virus's capsidthe protein shell covering the viral core (Figure 1). Our results show that viruses from different but closely related families can be differentiated by AFM on the basis of their capsid structure.

As a cell responds to its changing 作 exponded like ang compressed and Understanding the compaction expansion states of DNA is an important step toward detecting and treating diseases through biochemistry. Working with UC Davis, BSNL scientists use highinteracts with selected proteins. By adding varying amounts of Abf2p-a protein solution, the researchers have found that when this protein binds to the DNA, it produces a sharp kink in the otherwise
fairly loose, open DNA strand (Figure 2) Adding more protein produces more kinks-each kink angled roughly 102 degrees. When sufficient Abf2

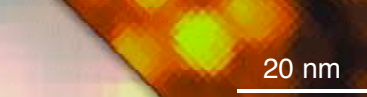

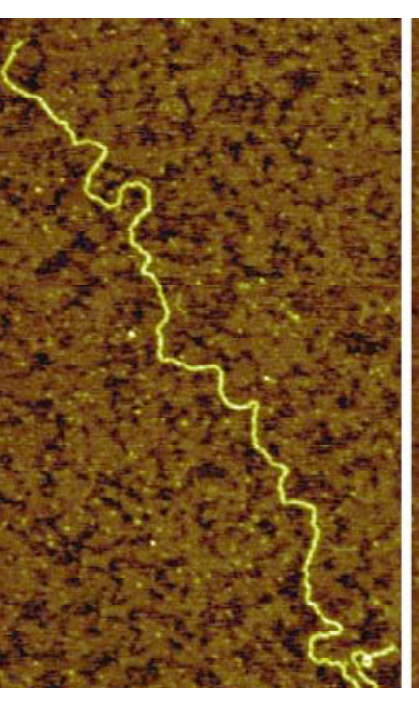
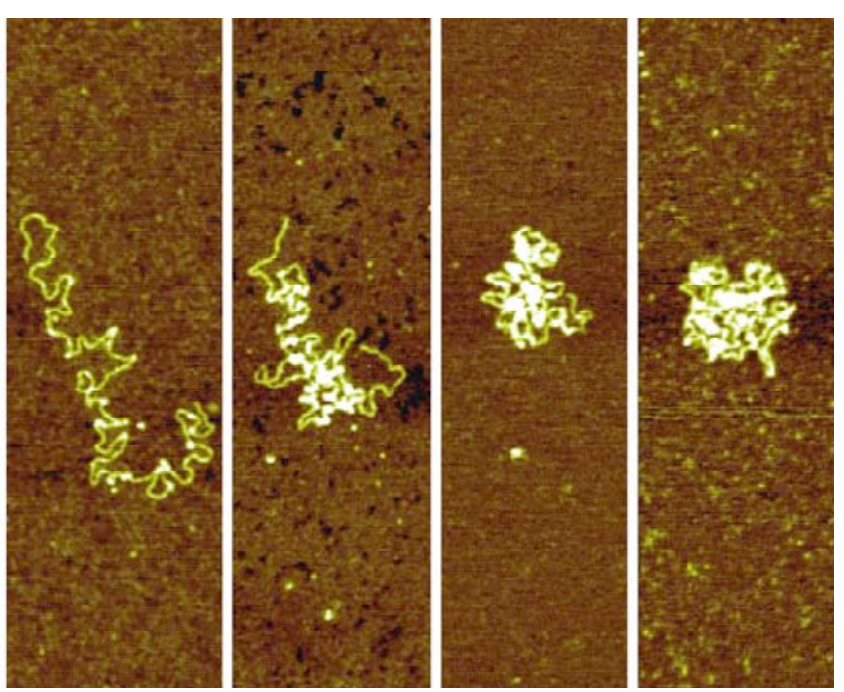

Figure 2. Progressive

AFM images show the
compaction of DNA

compaction of DNA

amounts of Abt2p yeast

protein. Understanding

ane compaction of DNA is

detecting and treating

diseases.

protein is added, the DNA collapses into a State-of-the-Art Technologies compact globular structure, which protect cause Parkinson's and other diseases. BSNL is also developing cutting-edge approaches in protein engineering techniques to study the affinity and selectivity of ligands as they bind to a specific site on a protein. New methods the drug his-aflinity ligands can aid in targeted drug delivery. Our afin take advantage of the powerful forces of selection and amplification from with the context of in vitro or whole-cell platforms that provide for a high degree control and certainty. Combined, these approaches should provide rapid access
high-affinity ligands for virtually any molecular target. Integrating these liga with other BSL science and research elements will allow us to develop nextenvironmental biology research, and human health applications. BSNL has brought together a unique suite of analytical tools that provide advanced characterization capabilities at the molecular to single-organism level. Our investment in single-molecule optic evelopment of ultrasensitive detectio nd also research into protein-protein

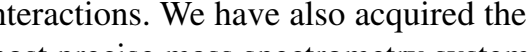
available for hish throunput prystens analysis and profiling chemical signa cross individual cells. Of particular note is the NanoSIMS (Secondary Ion Mass pectrometer) used for chemical isotope maging. This instrument is only one of wo in the country, with spatial resolution down to $50 \mathrm{~nm}$. We are using it to study (a) boremediation-and bacterial spores The to homeland security. aboratories can spatially determine a structure at the single-protein level, measure interaction forces at the singlemolecule level, and chemically pattern surfaces at 10-nm-length scales. These microscopy combined with a unique microfluidic chip to refine the singlemolecule measurement of protein-folding laboratories enable BSNL scientists to pursue a wide range of biosecurity an

\section{NEW FRONTE}

Prions cause several degenerative Porders such as Mad Cow disease, called scarpie, and variant CreutzfeldtJacob disease in humans. Its detection had been difficult until very recently when scientists discovered that prio proteins could be detected by an

Our team is developing techniques to detect prions in sheep blood serum In addition, we are using microfluidic channels and an optical microscope to detect fluorescence from the prions. This project involves a challenging and mportant technical task in chemical biology, and it illustrates very

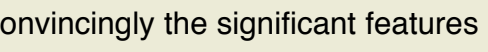
multiple, state-ofthe techniques and a multidisciplinary to address a problem that has thus far defied analysis. 


\section{Energetic Materials Center}

A national resource for research and development of explosives, pyrotechnics, and propellants

$T_{\text {he Energetic Materials Center }}$ (EMC) is operated jointly by the CMS and Defense and Nuclear Technologies directorates at Lawren
Livermore to conduct research and development on the performance of high as a core element of the nuclear weapo program, the EMC has grown to also support research and development for advanced conventional weapons, rocke and gun propellants, homeland secu denititization, and industial The EMC applies advanced theoretic computational, and experimental techniques to provide a detailed understanding of the chemistry and physics of energetic materials and their

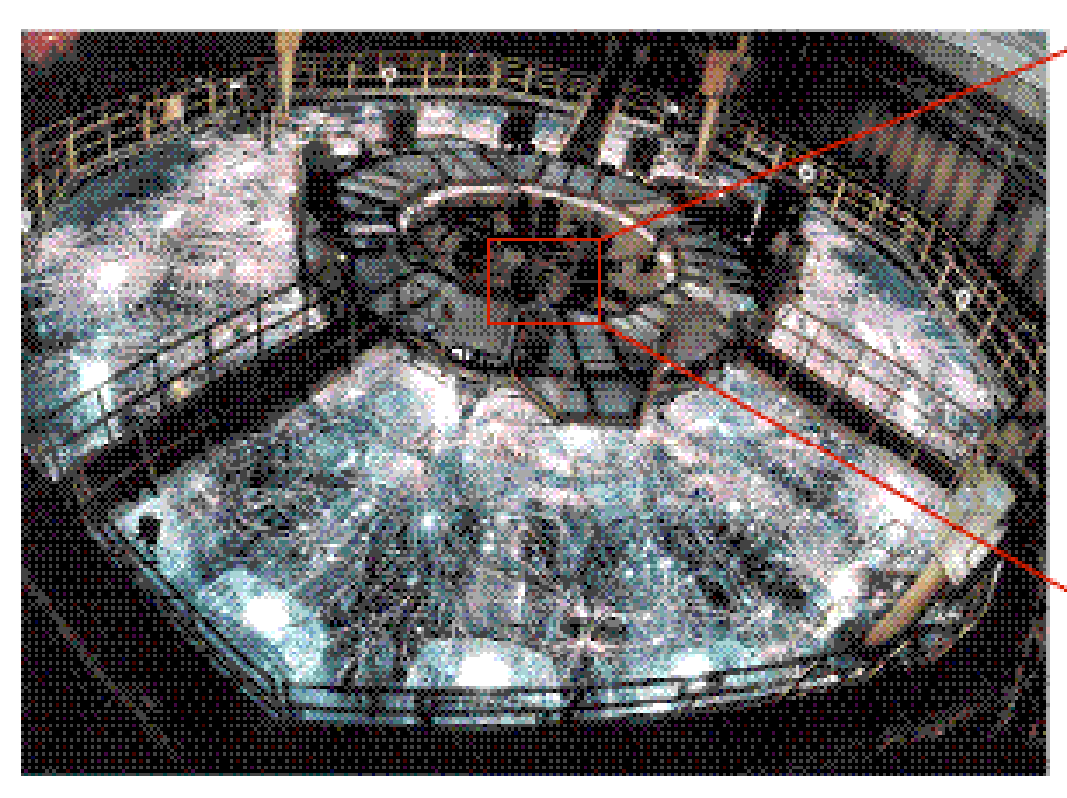

Figure 1. Using the Z Machine at Sandia National Laboratories, we have successfully obtained new measurements of explosives reactions at pressures up to $35 \mathrm{GPa}$. This image shows the Z Machine and four sample holders in the center. their perform

Strategic Theme-Related $\quad$ Z Machine at Sandia National $\begin{array}{ll}\text { Lemplishments in } 2004 & \text { Laboratories (Figure 1). These isentropic, } \\ \text { Research conducted by the EMC is } & \text { or shockless, experiments offer a new }\end{array}$

Research conducted by the EMC is aligned with the CMS theme of chemistry
under extreme conditions and chemical engineering in support of national-

security programs. of high explosives, we must first obtain their equation-of- wate information However, accurate data on their to obtain, as explosive materials react quickly to the extreme pressures typically
produced by strong shock waves in an tool to determine materials propertie without initiating cich
explosive samples.

We have also developed the capability to perform three-dimensional x-ray Explosives Applications Facility (HEAF) at LLNL to characterize the structure of internal voids inside bulk explosives. These voids have very significant influences on initiation and sensitivity of high explosives; however, their size applications to national defense, and the 政 properties at high pressures are difficult
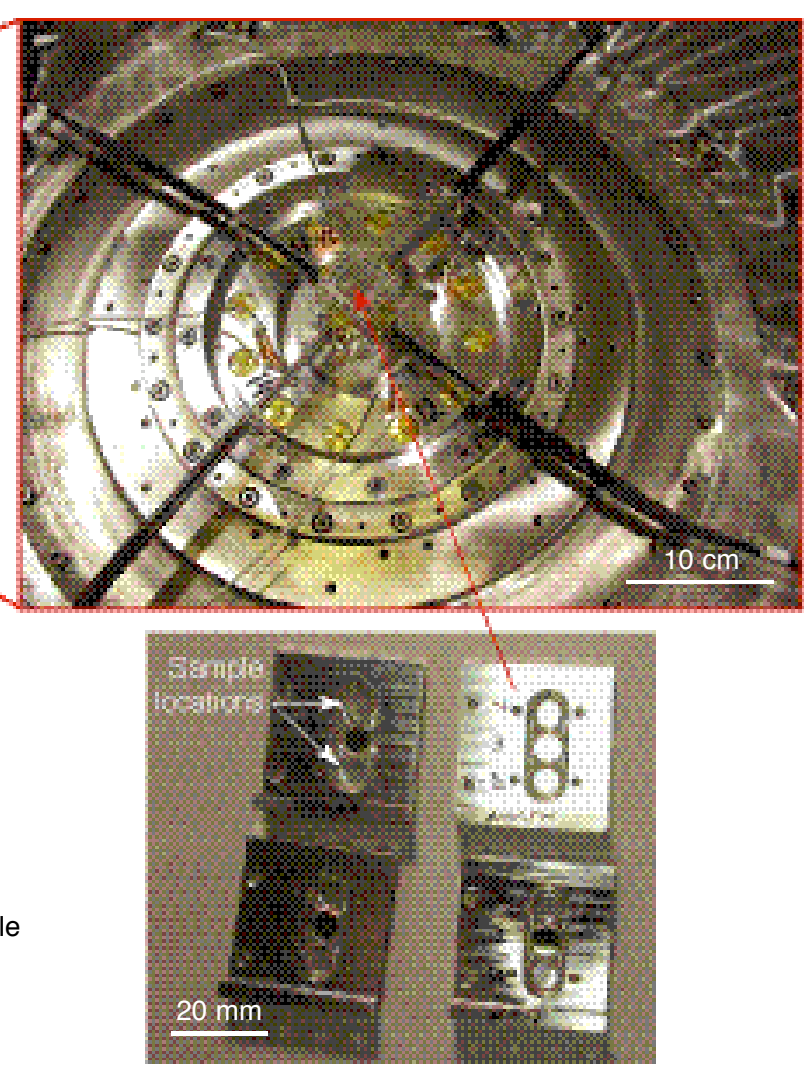

distributions have not been previously measured. This type of nondestructive analysis produces a map of the material density throughout the volume of explosive and can be particularly valuable in determining the location of inhomogeneities and voids resulting as any possible effects from aging wall thermal cycling. Our thermodynamics code, CHEETAH, is widely used as the premier high-explosives modeling environment. the latest release in 2004, Version 4 incorporates detailed chemical kinetic models for combustion of RDX and TN two common high explosives. Together are using computational quantum chemistry methods in CHEETAH fundamental properties of energetic molecules. Knowledge of these prop can be critical in predicting materials behavior, which can change dramatically over the range of pressures and

temperatures of interes. For example, our theoretical predictions indicate that the explosive volume reduction when the pressure reaches approximately $11 \mathrm{GPa}$ X-ray diffraction experiments at the Lawrence Berkeley National Laboratory's Advance Light Source have confirmed this

prediction.

In our quest to discover novel materials, we continue to conduc in which onal stutis of hybrid fullerenes, in a fullerene me allom by nitrogen atoms. We investigated the condensed phase properties of $\mathrm{C}_{48} \mathrm{~N}_{2}$ (Figure 2), showing that its ground-state solid has a face-centered-cubic structure and behaves like a semiconductor. While these computational studies were initially pursued as basic science research, our research has shown that these hybrid heterofullerenes are high-energy molecul
similar to explosives and may eventually

N L. MAIENSCHEIN (925) 423-1816, maienschein1@llnl.gov

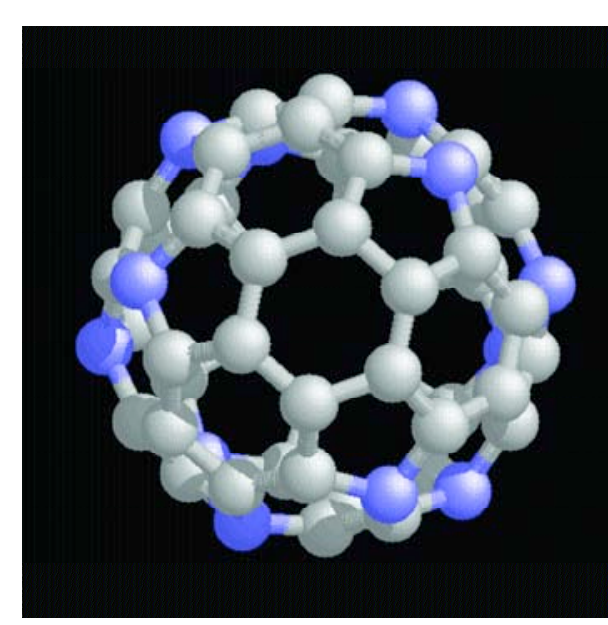

have a wide range of practical application

State-of-the-Art Technologies The EMC uses unique facilities to test high explosives and help understand and
control their chemistry. These energetic materials are characterized by nanosecon reactions that drive microsecond detonation processes and are tested i facilities including: - HEAF and Site 300, where explosives synthesis, formulation,
characterization, processing, and testing are conducted.

- The Contained Firing Facility at Site 300 and the Big Explosives Experimental Facility at the Nevada Test Site, where fully diagnosed explosives detonations are conducted. - The Forensic Science Center, where explosive detection methods are
developed in support of homeland developed in suppo
security programs.

In our dynamic experiments, we apply high-fidelity, high-speed diagnosticsincluding $\mathrm{x}$-ray radiography, high-speed photography, laser velocimetry, and embedded particle velocity and pressure gauges. The coupling of experimental results with multiscale simulations provides us with a unique ability to apply challenges posed by extopments to the
Figure 2. Hybrid fullerenes materials that behave like high explosives. Our computational structure of hybrid fullerene $\mathrm{C}_{48} \mathrm{~N}_{12}$ with carbon atoms in grey and nitrogen atoms in purple.

\section{NEW FRONTIERS}

The combination of cutting-edg computational analysis and highly advances in energetic materias research. We are focusing on four $m$ a categories: performance, safety, reliability/surveillance, and new materials. The new diagnostic tools we use to observe the propagation of a detonation wave will enable the development of improved tools to analyze the performance of existing and us to develop more effective application of insensitive explosives in the stockpile and in conventional weapons. Similarly this approach will lead to the ability to evaluate the response of energetic materials to a wide variety of stimuli, providing a scientific basis for resolving safety questions and improving designs with respect to safety.

We conthue to focus on detailed understanding of the aging effects in identifying potential age related changes and extending the projected lifetime of energetic materials. Finally, we are developing novel energetic molecules, formulations, and nanoenergetics to support improved safety and performance in the nuclear weapons stockpile as well as the trend toward small, high-value weapon systems that require innovative materials.

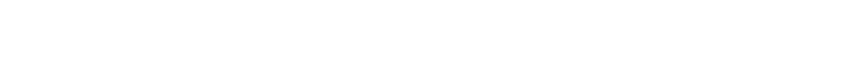




\section{Forensic Science Center}

Supporting national-security needs in chemical, nuclear, biological, and high-explosives counterterrorism

7 he Forensic Science Center (FSC) provides advanced analysis for Livermore's national-security prog
chemical, nuclear, and biological counterterrorism. While serving the immediate, short-term needs in these areas, the center also develops a technology portfolio to support long-term missions.

We are strongly engaged in international and national interests through a variety of federal agencies
including the Federal Bureau of Investigation (FBI), the Departme Investigation (FB), the Department of and Drug Administration. From these agencies, the FSC routinely receives unknown samples for analysis and identification. Our unique forensic capabilities contribute significantly in the nation's efforts to interdict dangerou materials and defeat terrorism. We leverage this expertise in a number of was to suppor estabrished in the areas of national security nonproliferation, and intelligence support Strategic Theme-Related Accomplishments in 2004 Our work at the FSC supports two CMS research themes: (1) science in support of national objectives at the intersection of chemistry, materials science, and biology and (2) applied nuclear science for human health and nalional security.

the Pronis certined by the Organtion for the Prohibition of Chemical Weapo implementing body of the Chemica Weapons Convention-to analyze for the possible presence of chemical warfare agents. To maintain certification, the FSC participates in ongoing proficien creates and sends samples to participating must corre ty alysis. Each laboratory dangerous ingredients to reman portied to obtain certification. tests in which one OPCW laboratory
In 2004, the FSC was asked to prepar and distribute samples. Nine different which were synthesized in the FSC and seven others obtained from commercia sources (Figure 1). The samples were "spiked" with chemical interference and reportable compounds that included chemical weapons precursors and degradation products at concentration levels ranging from 5 to $500 \mathrm{ppm}$ to represent a possible inspection scenario. stability tests and forensic analysis of the samples using several techniques that included nuclear magnetic resonance and gas chromatograph-mass spectrometry. The FSC is emerging as a critical scientific center for national security. In 2004, the FBI, in collaboration with the forensic networks that specialize in bronsic analysis. Tha and DHS acal funding the FSC is develophs and FBI protocols and entanced forensic analysis DHS, began the groundwork for a set of

Figure 1. As requested by the Organization for the Prohibition of Chemical Weapons, the Forensic Science Center prepared a set of samples for the ongoing proficiency tests,

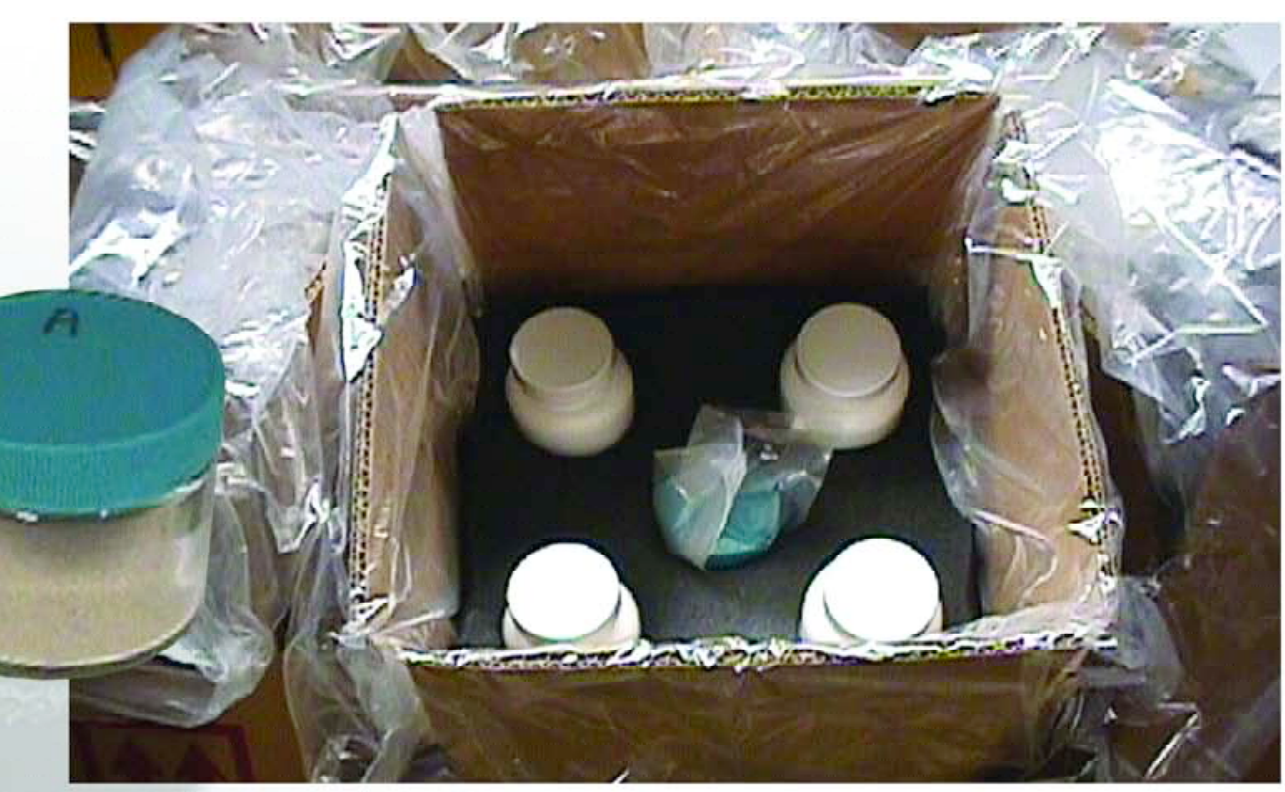

For more information contact

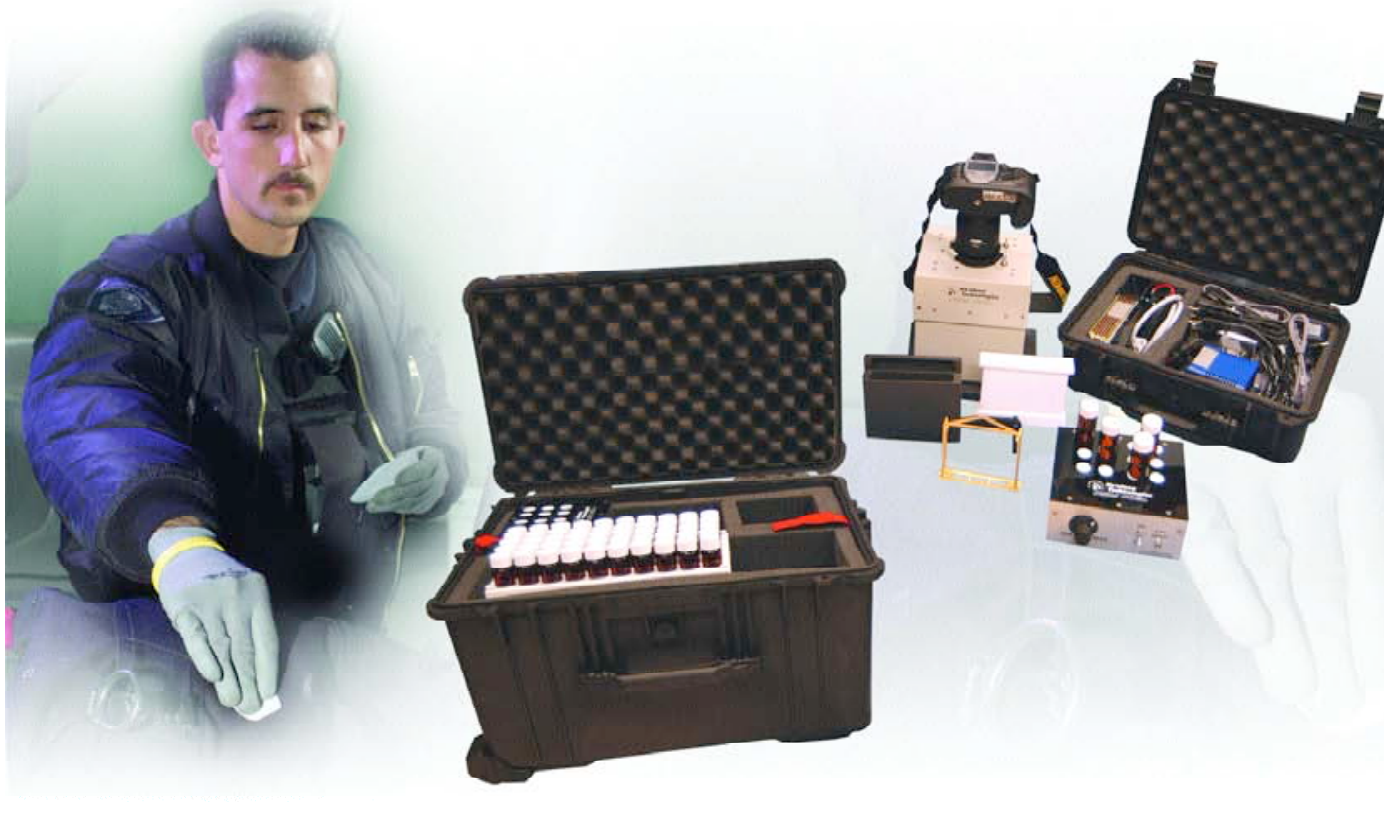

capabilities to support the growing national mis attribution. Our center has also been 作 the Food Emergency Response Network, a national sentine laboratory system that monitors and analyzes potential threats to the nation's

We also participated in TOPOFF 3the most comprehensive terrorism exercise involved 10,000 particip from more than 275 organizations in the United Kingdom, Canada, and the United States. The event simulated biological and chemical releases, potential huma injuries that might occur as a result, and response actions taken under such a scenatio. The FSC provided technical responders over a wide ra inquiries from arpes in semical and biological science and technology.

State-of-the-Art Technologies We are continually searching for new ways to combine analytical methods with challenging new applications, as well as packaging the tools into smaller, mo
sensitive configurations to provide portability and flexibility in the field. These compact instruments serve as mobile chemistry laboratories that greatly making.

During the past year, the U.S. Arm completed a validation test of a fielddeployable analysis kit developed by the F (Figure 2) uses thin-layer chromatograp (TLC) a technique previously only available in a laboratory. The new portable TLC kit enables a trained technician to test a sample immediately in the field, reducing the assessment time from days or weeks to minutes and avoiding transport of potentially commercial partner.

We bever a new of explosives using a chromometer. One of the greatest national-security concern is the utilization of explosives in improvised explosive devices. New technology designed at the FSC allows the rapid testing of over 30 different explosives and can be implemented by technology has now been transferred to gure 2. The Forensic Science Center has developed a fieldthin-layer chromatography (TLC) to assess the stability of munitions and propellants. Previously only portable TLC kit can reduce the assessment time from days or weeks to minutes and avoid

first responders with minimal training. The equipment has been field-tested in several "real-world" scenarios and has deployable systems.

\section{NEW FRONTIERS}

FSC scientists will continue to expand their capabilities in analytical science, instrument development, molecular and nanostructured materi synthesis. Future scientific theme areas include next-generation chemical and explosives detection, as well as bioforensics emphasizing chemical and physical signatures. Working with the Energy, and, FSC is strengthening programmatic connections that will use FSC and other unique resources at Lawrence Livermore. Our center is increasing its role as a resource for chemical, biological, radiological, and explosives expertise assistance and is poised to move rapidly to respond to the growing
needs of national security. 


\section{Glenn T. Seabory Institute}

Fostering research in fundamental and applied nuclear science and technology

7 he Glenn T. Seaborg Institute (GTSI) was formed in 1991 to provide scientific leadership in nuclea includes the development of advanced detection techniques to identify and characterize the effects of radioactive and nuclear-proliferation materials. Our research also contributes toward promoting a clean environment and assisting in the detection and treat
disease. Efforts at GTSI focus on: disease. Efforts at GTSI focus on:
- Science that advances the safety and reliability of the nation's nucety reliability of the nate
weapons stockpile

- Research to reveal new ways to counter terrorism and deter the proliferation of nuclear material Applying radiation science to improve

Scientific methodologies for assessing the vulnerability of the drinking-wate supply and for characterizing the testing

- Science education to enhance public understanding and student involvement in nuclear research

Figure 1. Transmission electron microscopy images of a dust particte
from Earth's upper from Earth's upper total energy emitted from the particle (top left) and the energy distribution for each of the elements in the particle.
The institute maintains a world-class Electron Microscope (STEM) and Soscale-resolution Secondary Ion Mass evoling national-security needs. In training and development of nextgeneration nuclear scientists by hosting interns and summer students from top universities around the country.

\section{Strategic Theme-Related} (in 2004 Research conducted by the GTSI align nuclear science for human health and national security. Spectrometer (NanoSIMS). In with the Institute of Geophysics and Planetary Physics at nanoSIMS to analyze interplanetary dust particles and determine if the particles were formed before or after the formation of the solar system. Researchers look for he abundance of specific elements sample and profliles of oxygen, features, all of which and sulfur on a particle's formation and evolution processes at the nanoscale level, GTSI utilizes an array of unique technologies such as the
200-keV Scanning Tunneling

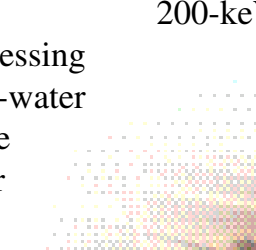

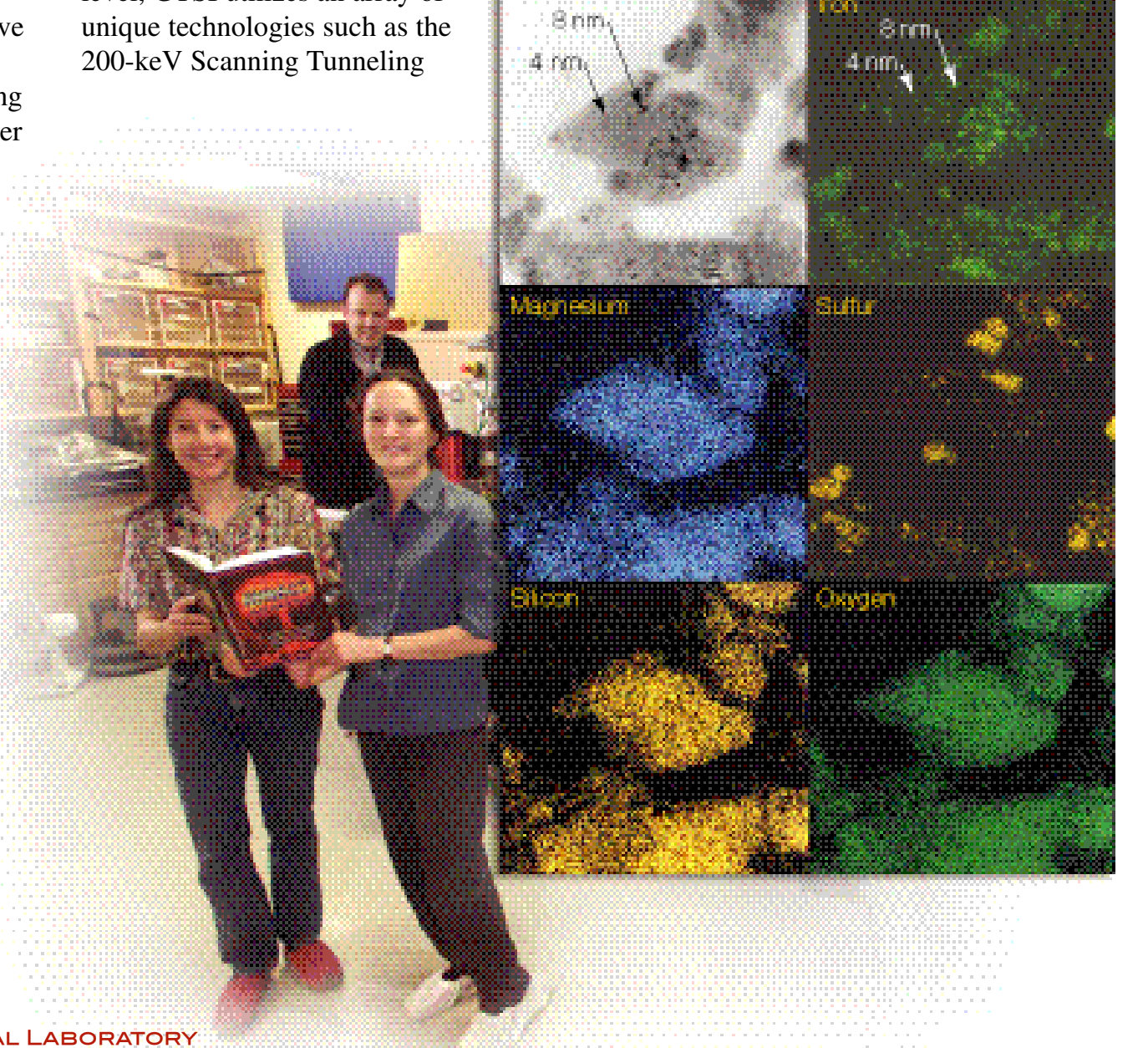
GTSI is contributing to biosecurity
efforts by using the nanoSIMS to identify elemental and isotopic signatures of anthrax and other dangerous pathogens. We are combining genetic typing with new, state-of-the-art bioforensics technology. We can, for the first time, fingerprint individual spores from a very of biological weapon agents. GTSI continues to develop new gamma radiation detection devices for applications ranging from biomedical research and nuclear medicine to national security and astrophysics. Recent efforts have been focused on Compton scattering-based inagers, which provide high source resolution and sensitivity. dimensional, high-purity germanium arrays to deduce the incident direction the gamma rays. While the main goal of this detector is finding clandestine nucle material, it could also be used with radiolabeled tracers to detect unique molecular characteristics of early cances. To improve the accuracy of dose determination in radiation therapy, we phantom-a human-body model used i calculating dosage delivery adjustable thickness. The Radiation Phantom with Humanoid Shape and Adjustable Thickness (RPHAT) image system will provide physicians the ability to adjust dosages and target delivery sites according to the weight of each patient. Figure 2 shows whe difference in radiation dose and dellvery stes for two prostate

Our expertise in isotope science also contributes to improving the environment, specifically in addressing the growing concern of the vulnerability of groundwater to contamination. For example, in California, which relies on groundwater for 50 percent of its water needs, the leading cause for the closure many drinking water supply wells is GTSI have been using its state-of the-at

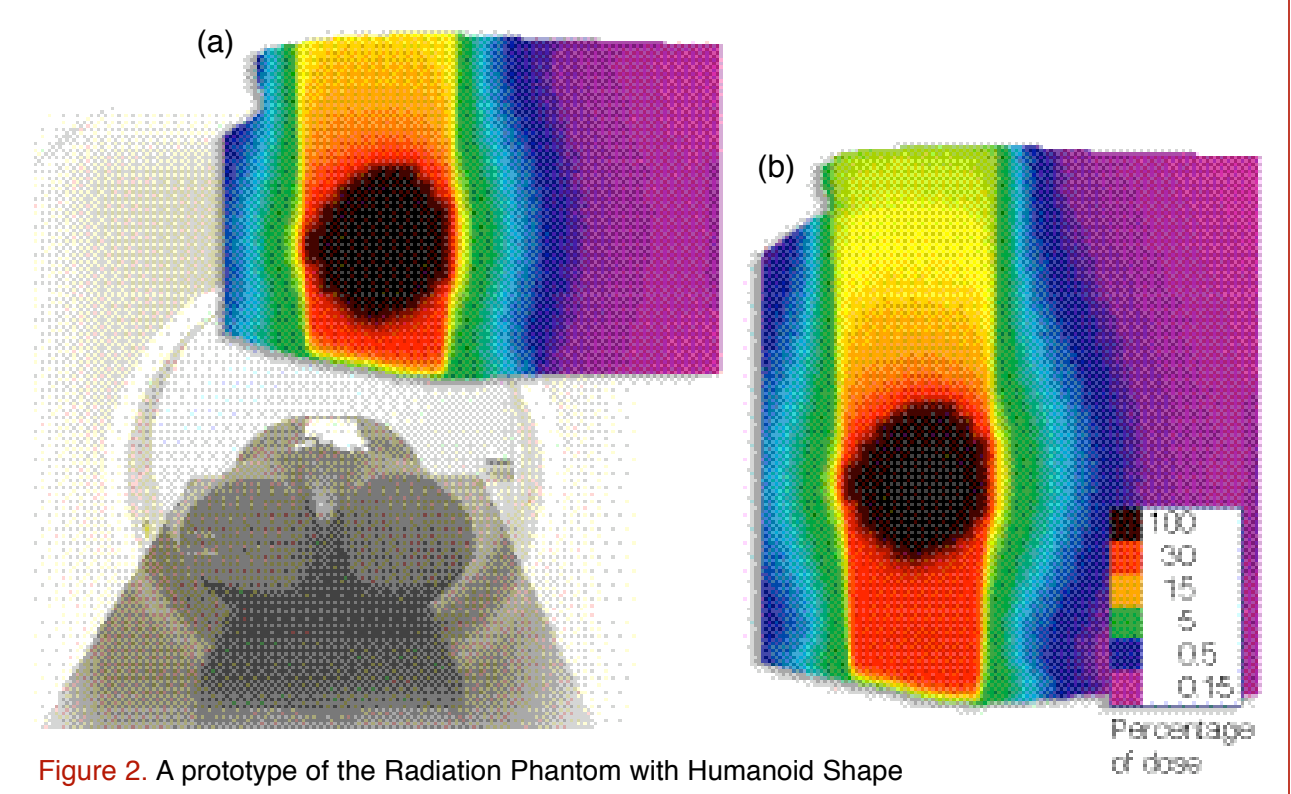

Figure 2. A prototype of the Radiation Phantom with Humanoid Shape and Adjustable Thickness, or RPHAT, model (botlom) is entering a CT thin radiotherapy patient (a) and a heavier patient (b) mass spectrometry capability to conduct studies using noble gas to determine which wells might be at risk for contamination.

An integral part of the success of scientists to ensure conting young basic and applied sciences. GTSI's Nuclear Science Internship Progra sponsored by the Department of Energy, provides nuclear science students wit individual mentors in nuclear and

analytical chemistry. During the summer of 2004, GTSI hosted 20 college gradua around

State-of-the-Art Technologies The GTSI utilizes a number of unique programmatic activities, and these technologies often contribute toward oth areas of basic science research. For

example, the STEM and nanoSIMS tools used in work for homeland security, nonproliferation, and national security astrophysics, new element discor nuclear medicine, and environmental research. Through their applications in new techisces forsts refine and develop also train yous for he wo facilities. We analytical tools to address problems ranging from attribution of elements in cosmology to solving water pollution Similarly, the Compton gamma detectors that are being developed for nationalnuclear medicine.

\section{NEW FRONTIERS}

Nuclear science represents a key enduring capability at LLNL. Our goal is nuclear and bionuclear science for important national problems such as homeland security, health, and the environment. We are also committed to recruiting and training young scientists pursuing careers in applied nuclear science so that we can ensure continuing discipline. 


\section{Center for National Security Applications of Magnetic Resonance}

Probing the structure and dynamics of molecules and materials for science in the national interest

The Center for National Security Applications of Magnetic besonance was created in 2003 to explore national defense challenges. The cen a multidisciplinary, state-of-the-art,
nuclear magnetic resonance (NMR) facility, housing instruments with unique capabilities and a strong research staff with diverse interest.

NMR uses large magnetic fields and radio-frequency spectroscopy to probe the structure and dynanics of molecules and resource for exploring the chemical, physical, and mechanical properties of biological, organic, and inorganic materials. Current projects include studies of sol-gel architectures for chemical synthesis and the structure of highmolecular-weight organic and inorganic complexes.

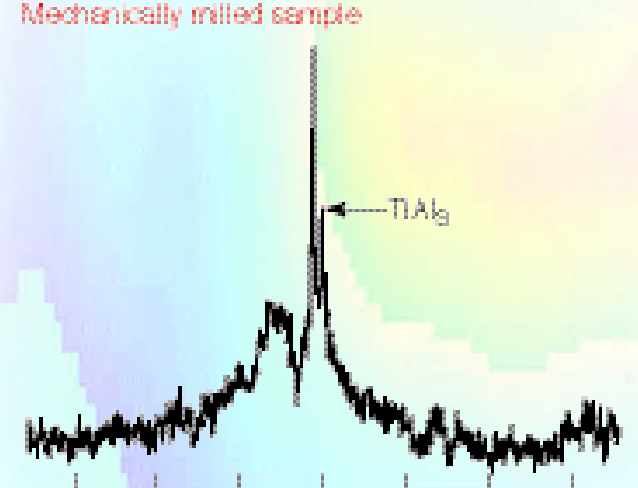

Strategic Theme-Related The wide range of fund analyses the center provides is aligned with all four of the CMS strategic theme areas. environments in situ without disturbing the processes in progress. Using NMR spectroscopy as an in situ characteriz tool, we saccessfully monitor particle materials in an elementally selective and nondestructive manner Aerogel materia synthesized using the sol-gel technique are becoming increasingly important in major LLNL programs. Unfortunately, detailed chemical processes that control the final aerogel morphology and properties are not yet known. That the 列

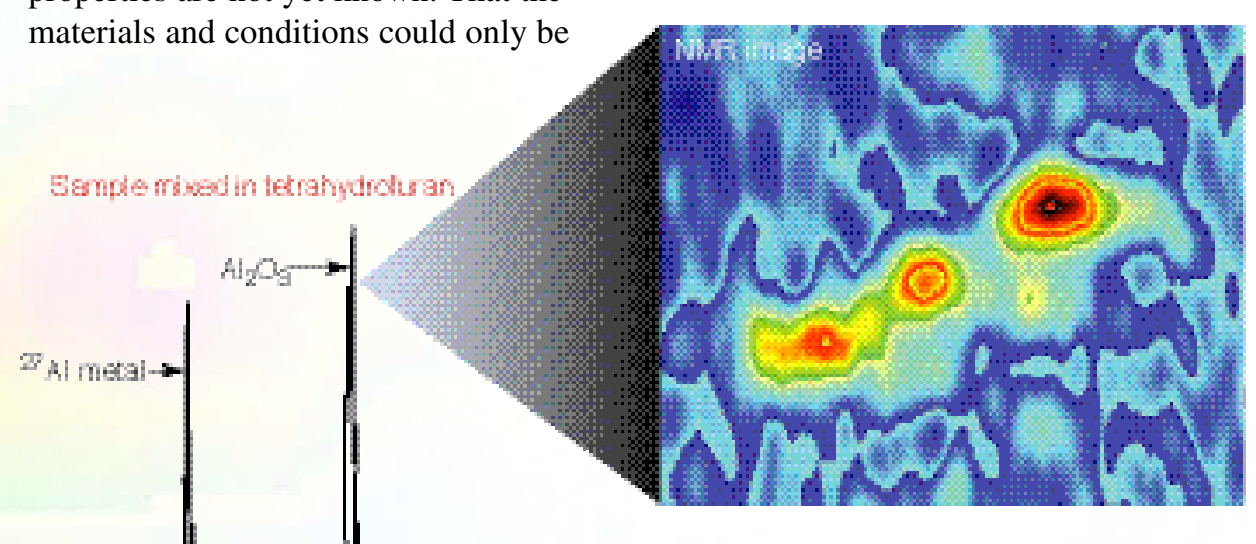

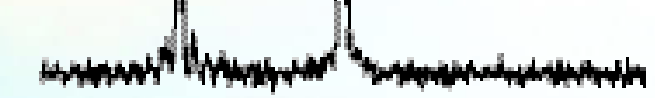
precursor, and extraction technique on the
One powerful feature of the NMR echnique is its ability to examine reacting observed and measured at the beginning

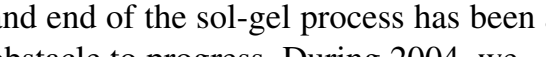
successfully probed the influences of such properties of aerogel.

To improve our understanding of hydrogen storage mechanisms, we have also been using a range of NMR techniques combined with computational modeling. Storing hydrogen using eiher titanitummetal-doped, carbon-based aerogels for new energy applications was observed to be known. Our NMR studies have provided a distinctive method of identifying the

Figure 1. Titanium doping enhances the hydrogen absorption and provides effective compounds in two heavily doped (33.3 at.\%) samples of hydrogen storage. NMR analysis revealed the formation of titanium

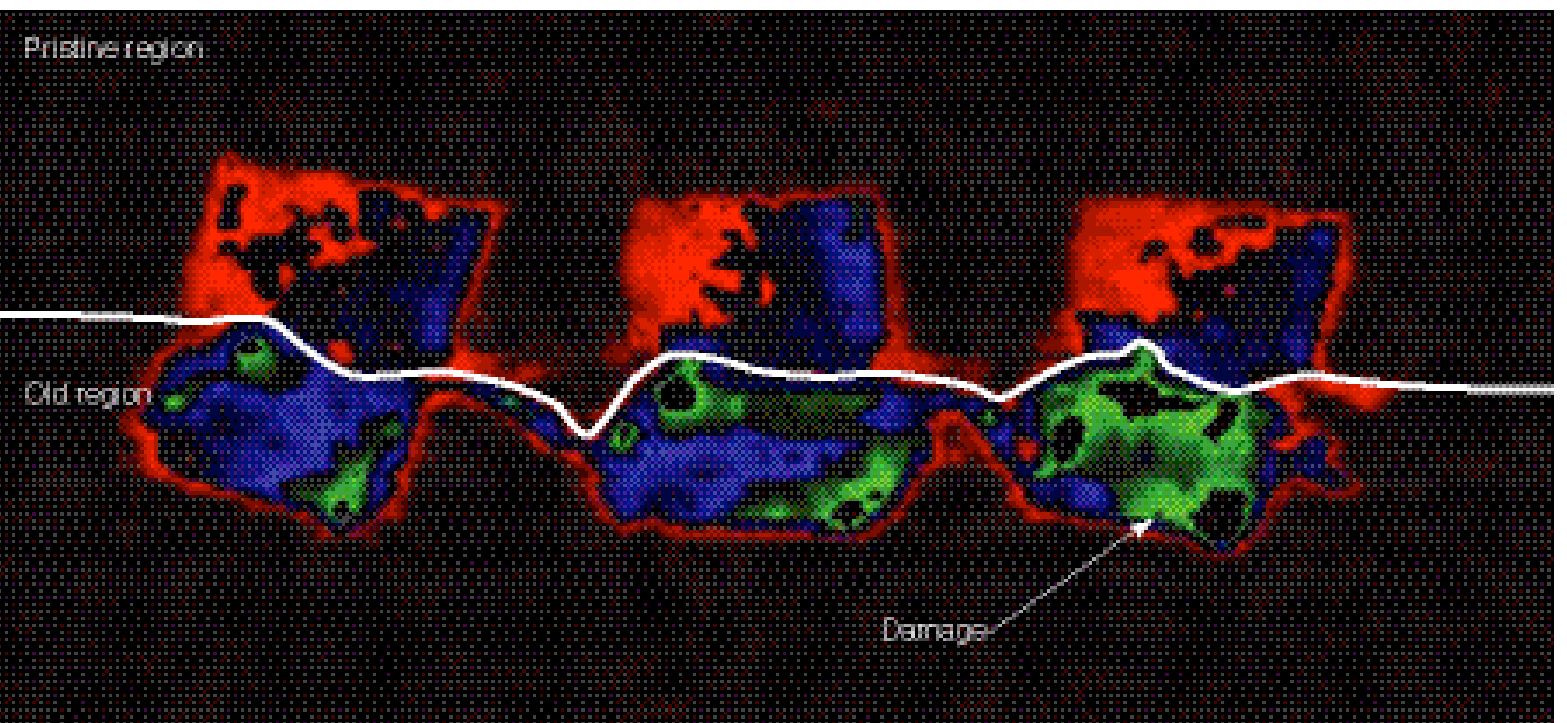

Figure 2. We employ magnetic resonance
imaging to compare and pristine section of a DC745 polym a non-nuclear weapons.

bonding and absorption of hydrogen in these materials. In collaboration with Sandia National Laboratories, we performed ${ }^{27} \mathrm{Al}$ solid-state NMR studies on the sodium-aluminum hydrides an explained how the titanium doping enhanced the hydrogen absorption (Figure 1). We are also beginning to apply our A major concern of the US. Stoger. Stewardship Program is how non-nucle components in weapons respond to lons periods of stress. For example, composite polymer materials with silica fillers can degrade when stored under extreme conditions. Ionizing radiation and therm degradation can cause changes of crosslink density and motional dynam of the polymers. Employing NMR nalysis as a diagnostic tool, we are scresles obticed from surveillarce NMR provides an excellent tool to probe radiation damage in the polymers. For example, we used magnetic resonance imaging (MRI) to create a spatial map of crosslink density in the polymers to dete damaged areas that are not normally
State-of-the-Art Technologies The center includes NMR spectrometers with operating frequencies of $300,400,500$, and $600 \mathrm{MHz}$. As partner in a northern California consortium, the center is acquiring a new, owerful 900-MHz spectrometer to be located a UC Benkeley. Oher capabilites include MRI and low-field, bench-top These facilities and the expertise are especially valuable to stockpile stewardship research and particularly to materials aging studies.

NMR is especially valuable because it can examine the material in situ, withou having to subject the material to

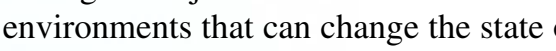
the material and invalidate the NMR is used in cach area of study, rerimental and computational tols to examine the evolution of the materials in question. In addition, using NMR expertise to integrate structural biology with materials characterization will provide a complementary resource of skills and knowledge that serves as a model of beneficial consolidation of facilities and scientists.
NEW FRONTIERS

The use of NMR to provide structural chemistry information about specific materials is well established. How we are now learning to use NMR techniques to provide key mechanistic formation about extremely complex chemical and physical problems that explanations. We are using NMR to study carbon evolution in soils and in the ocean to better understand the carbon cycle and possible opportunities for carbon sequestration. We are also studying the binding of biological molecules in a variely of applications in radio-immunotherapy, DNA damage, cancers, and bioterrorism. Many of continuing development of transportable NMR scanner with a radio-frequency coil that is less than $500 \mu \mathrm{m}$. The dramatically reduced size of such an instrument could provide a portable platform for detecting chemical and biological weapons and for imaging micro-organisms such as microbial 


\section{Nanoscale Synthesis and Characterization Laboratory}

To provide enabling capabilities for fabricating advanced nanoscale structures with novel properties

$\mathcal{Z}$ dvances in nanoscience and nanotechnology are poised to offer sweeping changes in the way we opt
materials and their functions. The Nanoscale Synthesis and Characterization Laboratory (NSCL) was formed in 2004 to create and exploit interdisciplinary research and development opportunities in nanoscience and nanotechnology. Through advanced synthesis, fabrication, and atomic-scale characterization, the NSCL will develop many new mater reaching potentials beyond our im farapplications in national security. The NSCL is investigating four science and technology areas: nanopor
materials, advanced nanocrystalline materials, three-dimensiona nanofabrication technologies, and nondestructive characterization. Among the diverse set of potential applications, our initial focus is to fabricate millimetesize target capsules for high-power laser hydrodynamic stability of target mate under extreme conditions.

Strategic Theme-Related
Accomplishments in 2004

Research at the NSCL aligns with the CMS research theme of materials properties and performance under is still very young, its ingug the NSCL is search team including new members from leading nanoscience groups in the country, has made significant advances in important technical areas (Figure 1).

Nanoporous Materials: Nanostructures with pore sizes less than $50-100 \mathrm{~nm}$ have many unique stractiral and transport properties that

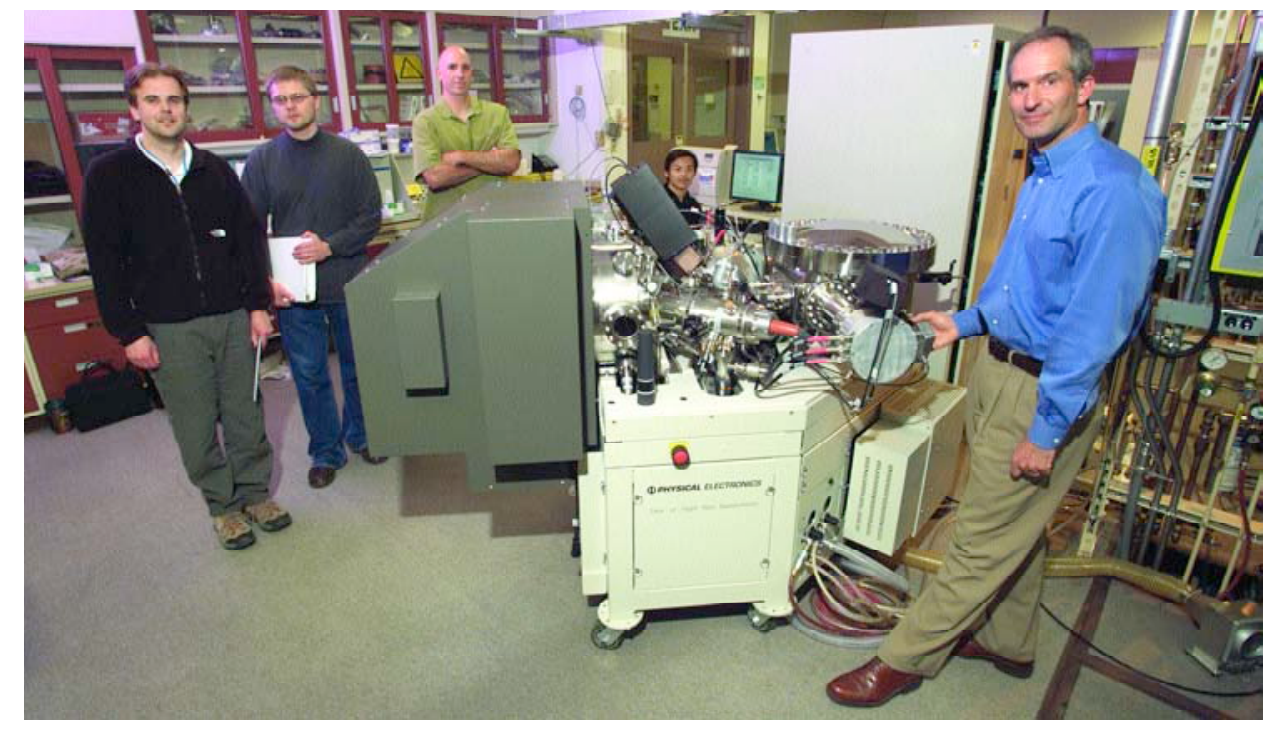

Figure 1. Attracting members from many leading nanoscience groups in the country, the newly formed Nanoscale Synthesis and Characterization Laboratory is already making significan scientific breakthroughs.

of modern technologies. Controlling the pore size and increasing the material
strength are the two key technologica challenges in making these materials. We successfully apply three different strategies for the production of nanoporous materials: dealloying, synthesizing low-density metal oxide aerogels, and depositing atomic layers on silica aerogel monoliths. Dealloying refers to the selective leaching or corrosions, whom alloys by structure with a lower density is produced. We used the dealloying of silver/gold alloys to produce gold metal foam at $7.5 \%$ of the original material density and with pore sizes as small $20 \mathrm{~nm}$ (Figure 2). Nanoporous gold normally displays a brittleness that $\mathrm{c}$
upon compression; however, when nanoporous gold foam is produced by dealloying, it deforms or compresses These unexpected properties may lead an important new class of high-yiead to strength, low-density materials ideally suited in target design for high-energydensity physics experiments. Another approach to synthesize lowdensity, nanoporous materials involves a new sol-gel process to produce titania (itanituris dioxide) acrogels with less than $10 \%$ of its normal density. Using highedge structure spectroscopy, we also characterized their structural and electronic properties. This new form of titania appears to have unique properties ideally suited for catalytic, photovoltaic, and gas sensor applications. By directly depositing layers of zincmicroscopy and $\mathrm{x}$-ray absorption ne

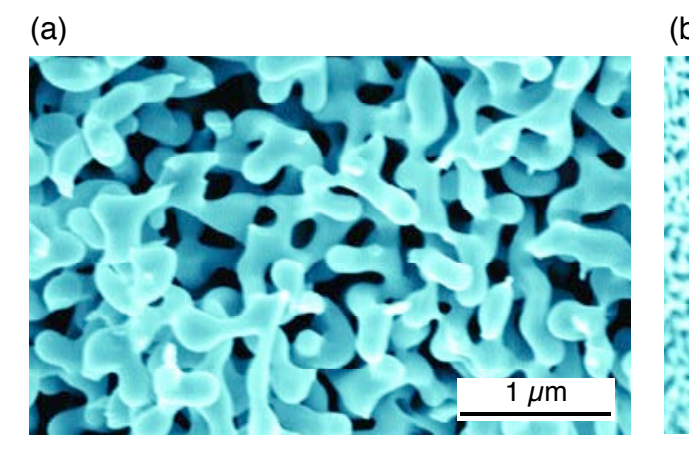

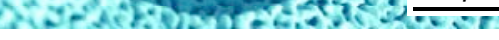
ving, we have successfully synthesized the first nanoporous gold foam with a pore size that is less than $100 \mathrm{~nm}$. (b) This new class of materials
exhibits novel properties. Under high strains, the gold foam is highly ductile and can be compacted by an indentation tip.

onto silica aerogels, we also produced a new class of acrogels that have a high new aerogels exhibit quantum confinement and other unique electronic and physical properties.

Nanocrystalline Materials: We produce the first-ever samples of nanocrystalline gold by compacting gold-metal fo produced by dealloying. During compression, the nanocrystalline charac of the foam is retained all the way to full and hardness nearly five times greater than the original polycrystalline gold.

Advanced High-Strength Metals: In 2004, we synthesized the first samples of nanocrystalline tantalum. Using transmission electron microscopy an nano-indentation to characterize its hardess and strength, we found uls mechanisms.

In other studies, we used plasmaassisted chemical vapor deposition to produce a thick $(100 \mu \mathrm{m})$ diamond film that may have advantges over beryllium coatings (Figure 3). The diamond film is an extremely strong surface coating, coating.
Nanofabrication: We have investigated varying from low-density conterials full-density metals, using computational carbon foam was found to produce a smoother but still highly porous surface, in contrast to ablating copper, nickel, an a a cor will be critical in lamete-size targes experiments.

Nondestructive characterization: We are using atomic-force microscopy to probe the structure of bonds between surfaces disparate materials, as part of a larger complex bonding processes. Also in 2004, we began to assess the potential of highspatial-resolution $\mathrm{x}$-ray imaging a objects.

State-of-the-Art Technologies In addition to a multitude of tools, the NSCL will use a newly installed dynamic transmission electro microscope with its ultrafast electron and experimental tools. Laser ablation of

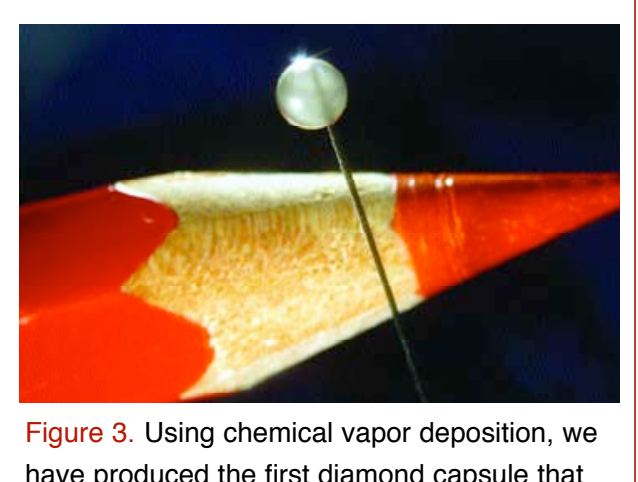

have produced the first diamond capsule that is ten times harder than the conventional beryllium materials.

with leading nanomaterials scientists on national user facilities such as the Advanced Light Source at Lawrence Berkeley National Laboratory, the Advanced Photon Source at Argonne National Laboratory, and others

\section{NEW FRONTIERS}

The advent of novel nanostructures wh ax wardnaty properies is leading enable many revolutionary applications. The NSCL intends to fabricate nanomaterials with characteristics that will meet the emerging requirements of our mission. In order to understand and control every step in the synthesis of these predictability in and to establish prediclabily in a wide range of apser target fabrication. We expect advances in nanoscience at Lawrence Livermore to significantly impact the future development of nano-celluar materials and nano-structured materials. 


\section{Postdoctoral Fellows and Graduate Students}

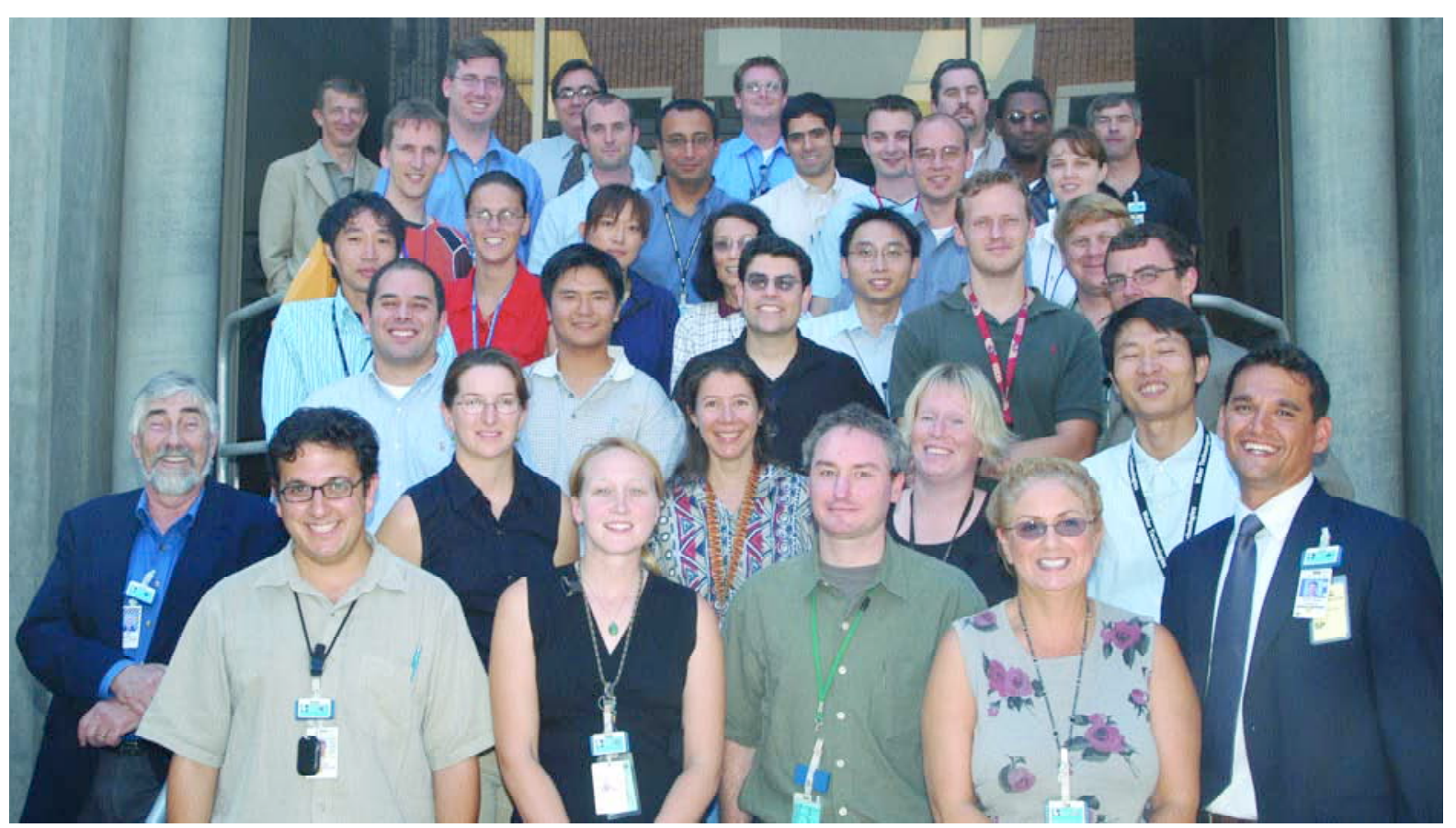

The CMS Postdoctoral Program continues to attract excellent young
cientists to Lawrence Livermore. They come from top universities around the world, bringing fresh ideas and leadingedge skills to our community. Many of our postdoctoral fellows are ma Laboratory programs and carrying out
excellent basic science research, while still maintaining close ties to externa collaborators. Approximately 40 postdoctoral researchers in CMS conduct work within a wide range of scientific disciplines. There are also about 15 graduate students pursuing degrees with CMS, each under the supervision of advisor. The postdoctoral and graduate students, grouped according to our current strategic research areas, include the following:

Nuclear Magnetic Resonance Anh-Tuyet Tran (UC Davis) is working on biological applications of nuclear magnetic resonance, particularly on chemical synthesis of adducts formed by DNA with heterocyclic amines-the carcinogens present in cooked foods and cigarette stmoke. Graduate student And Paravastu (UC Berkeley) is studying

\section{Nanoscale Materials Science and} chnology

Robert Meulenberg (UC Santa Barbara) is studying materials that are highly luminescent in the infrared by using rare-earth (lanthanide) doping on group IV nanocrystals. Raluca Negres (Central Florida) is studying defectreactions associated with laser-induced Yamage in "Mocis" Whang (Jystals. is working on deformation mechanisms of nanocrystalline materials and synthesis techniques for fabrication of highdensity experimental targets for the (UC Davis) is using small-angle x-ray scattering for determining nanostruct evolution of electronic structure in subNational Ignition Facility. Trevor Willey nanometer diamond-like carbon (dramordoids) and using near-edge $x$-ray astroctroscopy to exape Federal Institute of Techarange (Swiss working on nanoscale materials to provide high-energy-density experimental targets.

Nuclear Materials Stewardship Bassem El-Dasher (Carnegie Mellon) and Jeremy Gray (UC Davis) are studying the phase stability and crystalline interface behavior of the welds to be utilized in the Yucca Mountain wast contahnent outce bartier. Bassem Award from the American Society fow Metals for a paper he co-authored and was published in the journal, Metallurgical and Materials Transactions.

\section{Correlated Electron Systems and}

Alloy Properties
Tom Trelenberg (Florida State) has been studying formation of thin films and nanocrystals by laser ablation and subsequent thin-film characterization Sung Woo Yu (Universität Bielefeld, Germany) is using spin-resolved photoemission spectroscopy to investig ectron correlations in complex systen David Clatterbuck (UC Berkeley) of the phonon dispersion curves and studying phase transformation behavior plutonium using synchrotron radiationbased methods. Martin Butterfield (Loughborough, U.K.) has been carrying out spectroscopic studies of actinides, an Scott McCall (Florida State University) has been searching for a quantum critical
point in plutonium/americium alloys with low-temperature experiments on lowmagnetization, resistivity ant such heat. Michael Armstrong (University Rochester) is working on applications of the dynamic transmission electron microscopy for ultrafast diagnostics.

\section{High-Performance Computing} Masato Hiratani (Michigan Tech) is carrying out stochastic computer various conditions dynamics un annealing and incorporating analysis toots such as pattern analysis and chaos and fractal theory. I-feng Will Kuo (UC Irvine) has been carrying out classical quantum mechanical supercomputing studies of bulk and interfacial properties of water and selected problems in biochemistry. Aaron Golumbfskie (UC Berkeley) has been simulating comp biochem Goldman (UC Berkeley) has used firstprinciples molecular dynamics to study superionic phases of simple molecules, including water. Lawrence Fellow Evan Reed (MIT) is using computational and theoretical techniques to study optical emission from shock waves in crystals and developing multiscale simulation
methods for shock waves.
Biophysical and Interfacial Science The Biosecurity and Nanosciences Laboratory and other biophysical resear areas currently have the greatest number of postdoctoral researchers in CMS, reflecting the emphasis on Laboratory other discretionary funding sourees for this subject area. As part of the Physical Biosciences Institute, Amy Hiddessen (University of Pennsylvania), Chris Jea (Imperial College, U.K.), Ted Laurence (UCLA), Nan Shen (Harvard) and Tod Sulchek (Stanford) moved into CMS from the Biosciences Directorate during the past year. These researchers are working on a vare ry of biochemica interactions ping single-molecule detection. Marco Plom (University of Nijmegen, The Netherlands) is using atomic force microscopy to study the nanoscale structure of viruses and spores. Julie Smith (University of Western Australia) is using the NanoSIMS to study chemica signatures in biological weapons, early composition of extrant the isotopic Michael Stadermann (University of North Carolina) is investigating the use of carbon nanotubes in gas chromatography and as transducer elements in chemical sensors, and Jason Holt (California Institute of Technology) is developing carbon nanotube-based permeable membranes for a variety of separation processes such as desalination and naysis to understand the fundamentals of (Cambridge, U.K.) is using x-ray absorption spectroscopy for structural studies of biomimetic inorganic crysta growth on organic templates, and Richard Kimura (UC Davis) is developing methods with protein-splicing tools for biosynthesis of cyclic peptides containing cysteine knots. Brian Di
(University of Alberta, Canada) is studying thin films and microfabrication. condi (Carnegie Mellon) is arestation of the biominer of calcium phosphate modifiers. Youngeun Kwon (University of Chicago) is develoning protein microarrays by using chemical synthesi of proteins and engineering of biologically expressed proteins. Sapho Hok (UC Davis) is using dynamic combinatorial chemistry for target-driven ligand development to identify synthetic high-affinity ligands for tetanus toxin $C$ fragments. Tim Ratto (UC Davis) is using molecular recognition force nanoscale, investigating the adhesive properties of bacterial spores to decontamination materials, and designing fluidic membranes for protein production and analysis.

\section{Other Programs}

Thaddeus Norman (UC Santa Cruz) is using the diamond anvil cell to carry ou pressures. Keith Coffee (UC Riverside) is part of the BioAerosol Mass

Spectrometry program, studying the feasibility of using a combination of aerosol particle size, fluorescence, and mass-spectrum signatures for detection and identification of harmful biologica University) is working on groundwater David Campbell (Florida State) is examining the challenges inherent to mobile radiation detection systems for homeland-security applications. Additionally, he is engaged in the refinement of pulse-shape analysis algorithms for segmented high-purity germanium detectors. 


\section{Awards and Recognition in 2004}

Professional Society Honors 2 Bryan Balazs, Deputy Enhanced Surveillance Campaign Leader, received the 2004 Petersen Award from the Chemical Society. The Petersen Award presented each year at the section's annual awards meeting, recognizes a section member with the most cumulative service to the local group. Patrice Turchi, a CMS physicist, ha been elected as chairman of the Electronic, Magnetic, and Photonic Materials Division of the Minerals, serving as vice-chairman for more than serving as sice
two years.

Craig Tarver, a scientist in the extreme chemistry capability area; Lou Terminello, materials program leader for LLNL's Defense and Nuclear Technologies directorate; and David Eaglesham, former deputy associate director for science and technology, became Fellows of the American Physic contributions to shockwave physics in particular his developme implementation of the ignition an growth model for reactions in energetic materials and the non-equilibrium ZND theory for detonating energetic materials." Terminello was recognized "his innovative use of synchrotron radiation spectroscopy nin revealing the election a new "his seminal discoveries and technicol leadership in semi-conductor crystal growth and structural defects in epitaxia $\sum^{n}$ materials."

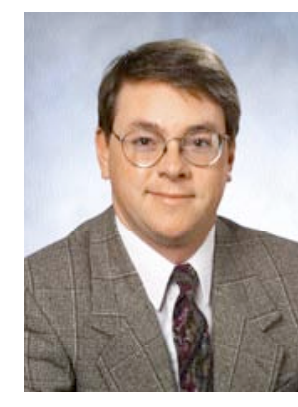
Bryan Balazs

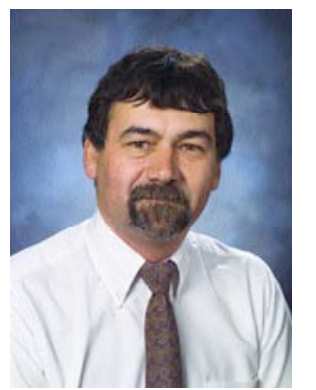
Patrice Turchi

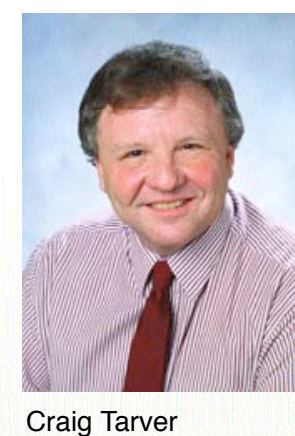

Craig Tarver

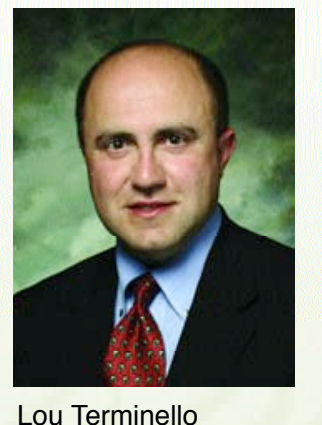

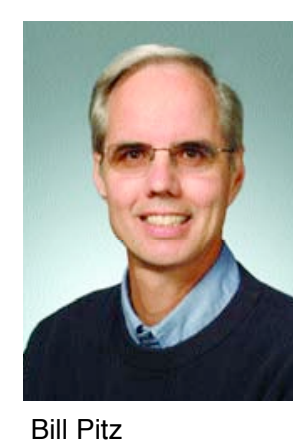

In 2004, Bill Pitz and Charlie Westbrook were notified of winning the Acch T. Colwe Arch paper, "Effects of Oxy gentes on Soor

Processes in DI Diesel Engines:

Experiments and Numerical Simulations," was written with four coauthors from was deemed the best among 2,492 papers
published for meetings of the Society of published for meetings of the Society of
Automotive Engineers during 2003 .

Scientific Achievements
LLNL Director Michael Anastasio LLNL Director Michael Anastasio
presented the fifth annual Science and presented the fitth annual Science and
Technology Awards to two teams of scientists. Jerry Britten led the team that developed the processing methods and tooling that produced both the world's largest multilayer dielectric reflection grating and the world's highest laser
damage-resistant gratings. This damage-resistant gratings. This
technology provides optics for hightechnology provides optics for highbreakthrough was made, no gratings were available for any of these lasers. Fellow CMS employees on the team included Leslie Summers. Anthony Van Buuren Jerry Britten

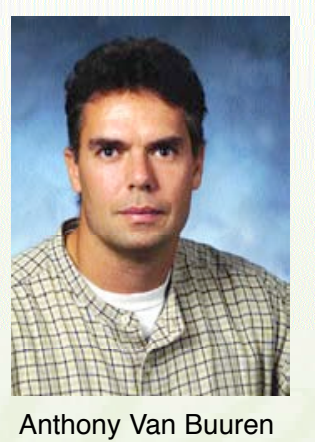

bucky-diamond and unraveling the atomic

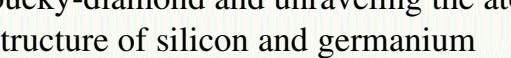

nanoparticles. The team's research focuses

how the properties of materials chang

Among the American Institute of

Physics (AIP) top physics stories for Sandia National Laboratories. Their work James Peterson, Curly Hoaglan, and was a member of the second team that

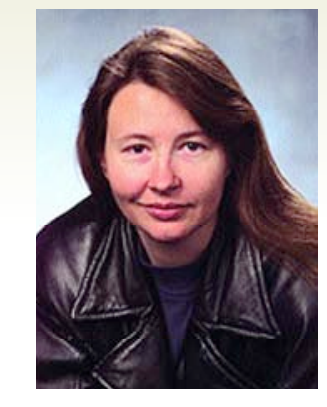
participants included Jackie Kenneally, Ken Moody, Mark Stoyer, Josh Patin, Ron Lougheed, Dawn Shaughnessy, John Wild, and Nancy Stoyer

Leadership Positions acquisitions coordinator, started a fourthe Ala

Editorships

societies, with about 13 CMS researchers serving as editors or on the editorial

boards of technical journals. These scientists provide a valuable con universities, other national laboratories, and important industries.

\section{Patents and Licenses}

participated in groundbreaking research

that resulted in a number of patents in

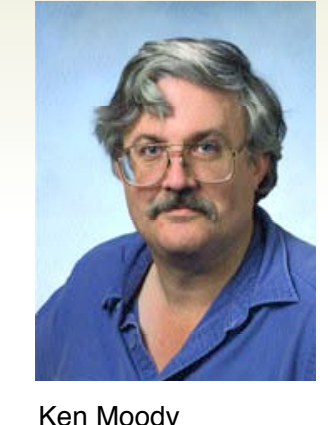

Ken Moody

2004, one Livermore project made the 115 at the Joint Institute for Nuclear Research in Dubna, Russia. CMS

Dick Quigley, a CMS excess

Many CMS scientists play important oles in their respective professional

CMS scientists and engineers

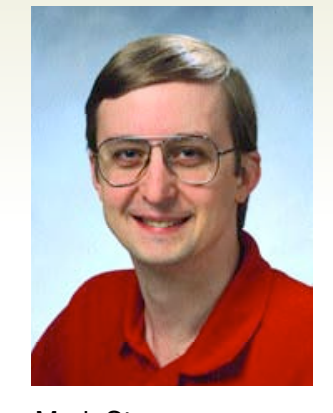

Mark Stoyer Josh Patin
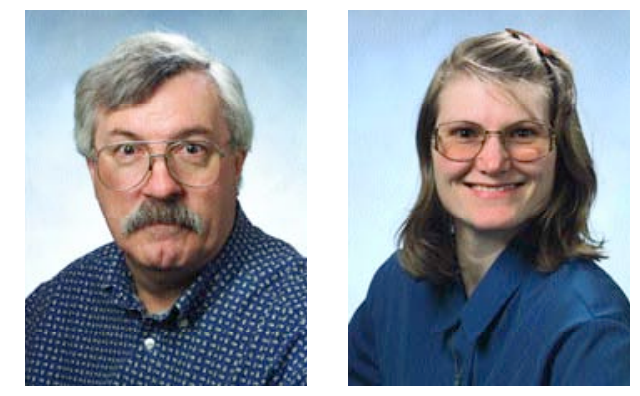

John Wild

Nancy Stoyer
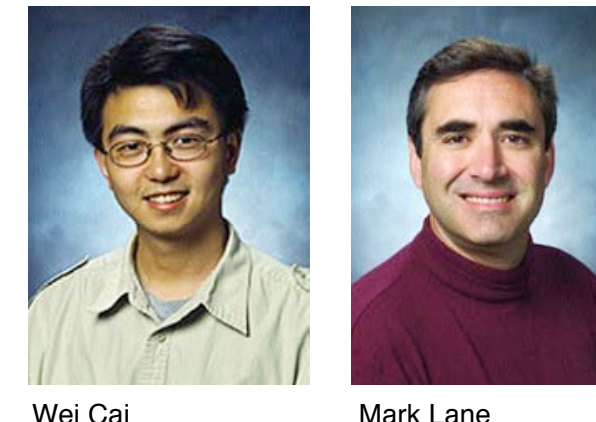

Wei Cai

Mark Lane
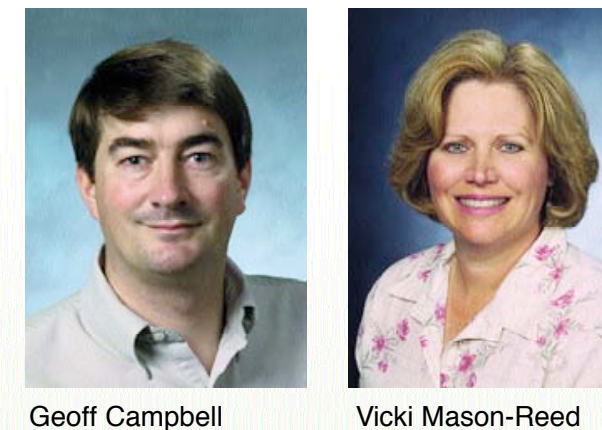

\begin{tabular}{|ll}
\hline Invention Disclosures & $\mathbf{3 4}$ \\
\hline Patent Applications & $\mathbf{1 9}$ \\
\hline Patents Issued & $\mathbf{8}$ \\
\hline Licenses Executed & $\mathbf{3}$
\end{tabular}

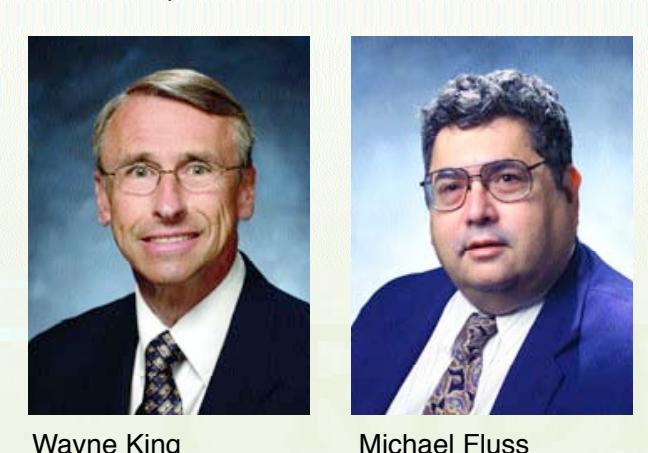

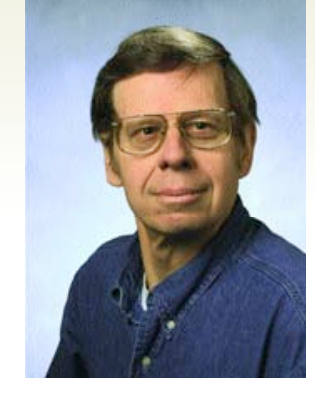

Ron Lougheed

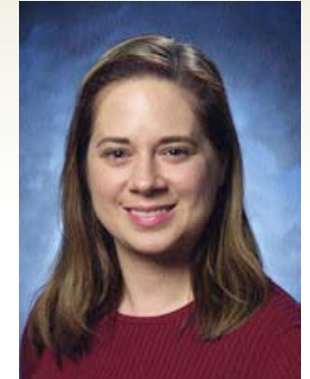

Dawn Shaughnessy

Postdoctoral Fellows

For a nersint pee Fellow Wei Cai, ngineering at Stanford University,

was named a Frederick E Terman Fellow.

The Terman fellowship program was established in 1994 with a gift from William R. Hewlett and David Packard. Other Recognitions

Mark Lane and Bryan Balazs of DOE Weapons Recognition of Excellence

Award The team was honored for their

work on an agressive project plan for

high-priority testing and evaluation of

existing and potential nuclear weapons

capability.

Geoff Campbell and Vicki MasonReed also received a DOE Weapons Recognition of Excellence Award. Geof and Vicki were members of the case new experimental platforms and a suite analysis tools and methodologies to study high explosives-driven radiation-case dynamics.

Wayne King, acting division leader Daterials Science and Technology Division, was awarded the Thiel College Distinguished Alumnus Award.

The CMS 2003 Annual Report received a Distignished Award in the Technical Communication internaty for competition. Stephanie Shang Charlie Westbrook, and Michael Fluss organized almost $30 \mathrm{CMS}$ contributions to the annual publication. 

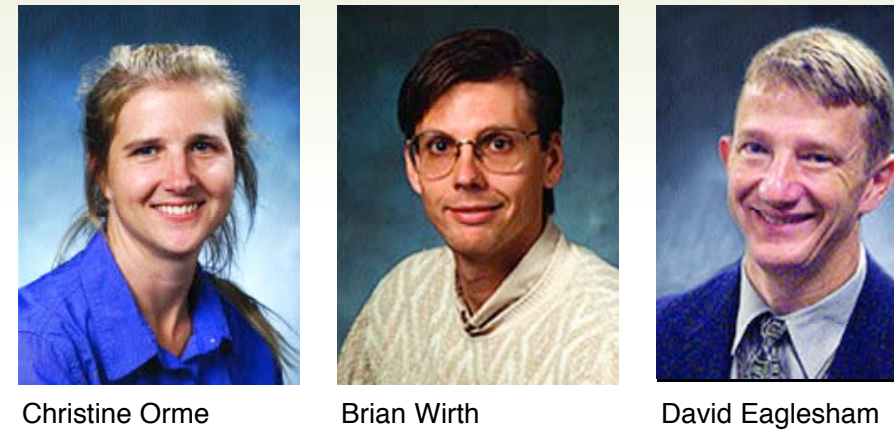

Two CMS scientists received the prestigious Presidential Early Caree Christine Orme and Brian Wirth (now a UC Berkeley faculty member) were recognized in a White House ceremo for groundbreaking accomplishments early in their careers and for their potential for future leadership.

University Collaborations

The research activities of CMS member with leading groups and individeractions

universities in the United States and other countries. Various university research programs have been identified as particularly desirable and valuable, leading CMS to provide funding to these program to facilitate interactions (for example, by granting graduate students and faculty special access to the Laboratory). There were more active collaborations in 2004 D. S.M. Yalso ye of Un David Eaglesham on implemed with characterization

Dr. Dudley R. Herschbach of Harvar University partnered with Carl Melius
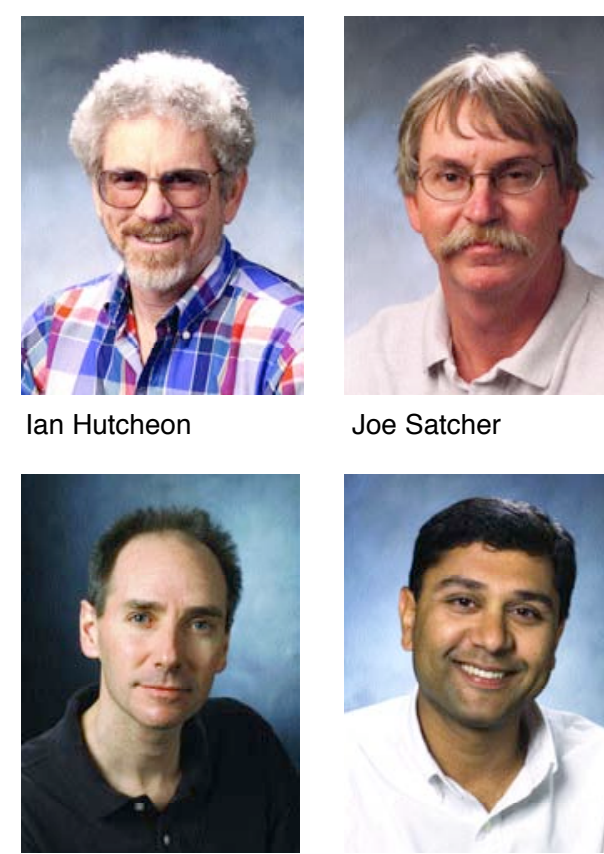

Christian Mailhiot

Mukul Kuma
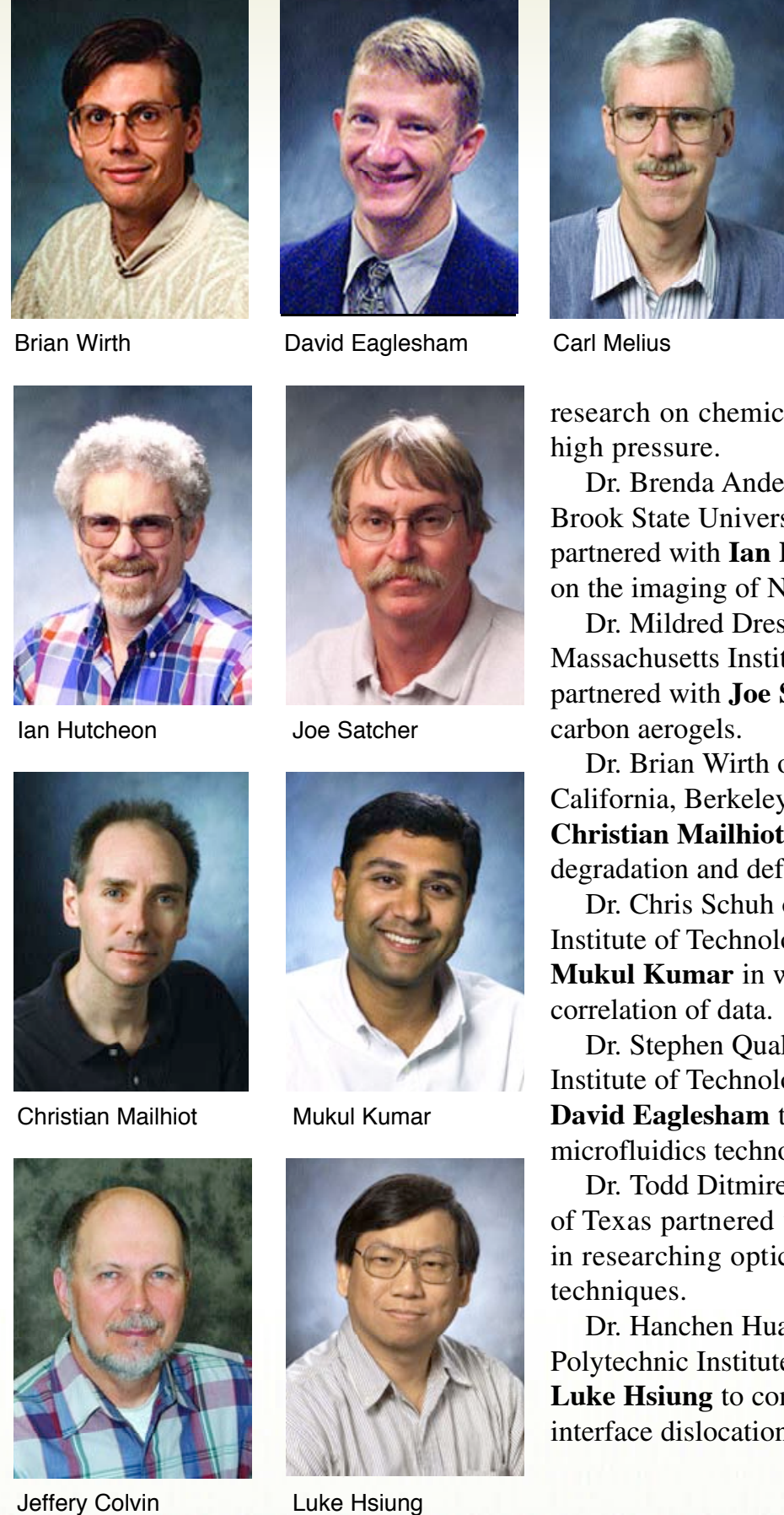

Carl Melius

research on chemical reactivity at high pressure.

Ding partned with In Hut of New York on the imaging of NanoSIMS. Dr. Mildred Dresselhaus of the Massachusetts Institute of Technology partnered with Joe Satcher to study carbon aerogels.

Dr. Brian Wirth of the University of Christian Berkeley, partnered with degradation and deformation. Dr. Chris Schuh of the Massc Ditute of Technology partnered wisetts Mukul Kumar in work on a higher order correlation of data.

Dr. Stephen Quake of the California Institute of Technology partnered with David Eaglesham to work o Dr. Todd Ditmire of the Dh. Tosd Dimire of the University in researching optical dignosics techniques.

Dr. Hanchen Huang of Rensselaer Polytechnic Institute collaborated with Luke Hsiung to conduct simulations of Luke Hsiung
2004 R\&D 100 AWARD

Each year, R\&D Magazine recognizes the 100 most technologically significant products of the year. CMS scientists contributed to one of the R\&D 100 Awards for 2004.

AUTONOMOUS PATHOGEN DETECTION SYSTEM

Livermore researchers have been developing a new instrument that can provide a rapid early warning system for the detection of pathogens, such as anthrax. The develophention System,

The lectern-size APDS can monitor the air continuouly for biolo threat agents and analyze samples within an hour. As the APDS collects air samples, it runs them through two different identification systems to reduce the probability of false alarms. The first system

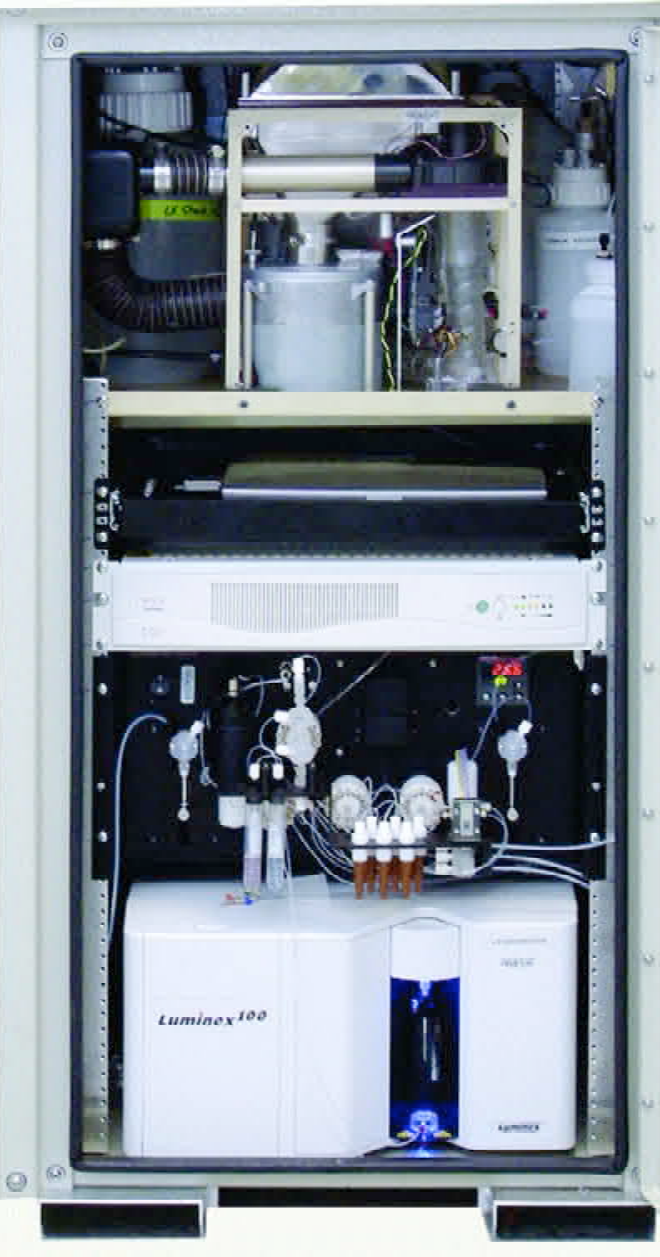

employs an immunoassay detector based on smalldiameter polystyrene beads, commonly known as microbeads, technology. A positive result from the first identification system triggers the second test, parich riss a separte andysis asing the DNA signatures of pathogens. Result from the system a

wirelessly every hour to a contronsmitted systems have been deployed at various location around the country, where they can provide continuous monitoring for up to seven days, unattended.

The Autonomous Pathogen Detection System monitors the air continuously for biological threat agents and uses two reducerton alarms itcen mability of false 100 different measure up to and reports identif per samp within an hour. 